\title{
The QUaD Galactic Plane Survey. II. A Compact Source Catalog
}

\section{Citation}

Culverhouse, T.; Ade, Peter; Bock, J.; Bowden, Melanie; Brown, Michael; Cahill, G.; Castro, Patricia; Church, Sarah; Friedman, R.; Ganga, K.; Gear, Walter; Gupta, Sujata; Hinderks, Jamie; Kovac, J.; Lange, Andrew; Leitch, E.; Melhuish, S. J.; Memari, Y.; Murphy, J.; Pryke, C.; Piccirillo, L.; Rajguru, N.; Rusholme, B.; Taylor, Andy; Wu, Edward; Zemcov, M. 2011. "The QUaD Galactic Plane Survey. II. A Compact Source Catalog." The Astrophysical Journal Supplement Series 195, no. 8: 8-37.

\section{Published Version}

doi:10.1088/0067-0049/195/1/8

\section{Permanent link}

http://nrs.harvard.edu/urn-3:HUL.InstRepos:12712868

\section{Terms of Use}

This article was downloaded from Harvard University's DASH repository, and is made available under the terms and conditions applicable to Other Posted Material, as set forth at http:// nrs.harvard.edu/urn-3:HUL.InstRepos:dash.current.terms-of-use\#LAA

\section{Share Your Story}

The Harvard community has made this article openly available. Please share how this access benefits you. Submit a story. 


\title{
THE QUaD GALACTIC PLANE SURVEY. II. A COMPACT SOURCE CATALOG
}

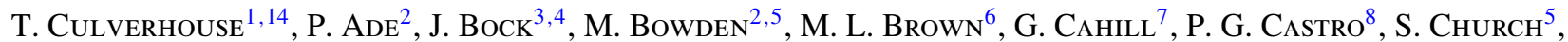 \\ R. Friedman ${ }^{1}$, K. Ganga ${ }^{9}$, W. K. Gear ${ }^{2}$, S. Gupta $^{2}$, J. Hinderks ${ }^{10}$, J. Kovac ${ }^{11}$, A. E. Lange ${ }^{4}$, E. Leitch $^{3,4}$, S. J. Melhuish $^{15}$, \\ Y. Memari ${ }^{12}$, J. A. Murphy ${ }^{7}$, A. Orlando ${ }^{4}$, C. Pryke ${ }^{1}$, R. Schwarz ${ }^{1}$, C. O'Sullivan ${ }^{7}$, L. Piccirillo ${ }^{15}$, N. Rajguru $^{13}$, \\ B. Rusholme ${ }^{16}$, A. N. TAYlor ${ }^{12}$, K. L. Thompson ${ }^{5}$, A. H. Turner ${ }^{2}$, E. Y. S. Wu ${ }^{5}$, And M. ZemCOV Th $^{3,4}$ \\ (QUaD COLlaboration) \\ ${ }^{1}$ Kavli Institute for Cosmological Physics, Department of Astronomy \& Astrophysics, \\ Enrico Fermi Institute, University of Chicago, Chicago, IL 60637, USA \\ ${ }^{2}$ School of Physics and Astronomy, Cardiff University, Cardiff CF24 3AA, UK \\ 3 Jet Propulsion Laboratory, Pasadena, CA 91109, USA \\ ${ }^{4}$ California Institute of Technology, Pasadena, CA 91125, USA \\ ${ }^{5}$ Kavli Institute for Particle Astrophysics and Cosmology and Department of Physics, Stanford University, Stanford, CA 94305, USA \\ ${ }^{6}$ Kavli Institute for Cosmology and Astrophysics Group, Cavendish Laboratory, University of Cambridge, Cambridge CB3 OHE, UK \\ ${ }^{7}$ Department of Experimental Physics, National University of Ireland Maynooth, Maynooth, Co. Kildare, Ireland \\ ${ }^{8}$ CENTRA, Departamento de Física, Edifício Ciência, Piso 4, Instituto Superior Técnico - IST, \\ Universidade Técnica de Lisboa, 1049-001 Lisboa, Portugal \\ ${ }^{9}$ Laboratoire APC/CNRS, Bâtiment Condorcet, 10, 75205 Paris Cedex 13, France \\ ${ }^{10}$ NASA Goddard Space Flight Center, Greenbelt, Maryland 20771, USA \\ ${ }^{11}$ Harvard Department of Astronomy, Harvard University, Cambridge MA 02138, USA \\ ${ }^{12}$ Institute for Astronomy, University of Edinburgh, Royal Observatory, Edinburgh EH9 3HJ, UK \\ ${ }^{13}$ Department of Physics and Astronomy, University College London, London WC1E 6BT, UK \\ Received 2010 May 20; accepted 2011 May 20; published 2011 June 29
}

\begin{abstract}
We present a catalog of compact sources derived from the QUaD Galactic Plane Survey. The survey covers $\sim 800 \mathrm{deg}^{2}$ of the inner galaxy $\left(|b|<4^{\circ}\right)$ in Stokes $I, Q$, and $U$ parameters at 100 and $150 \mathrm{GHz}$, with angular resolutions of 5 and 3.5 arcmin, respectively. Five hundred and twenty-six unique sources are identified in $I$, of which 239 are spatially matched between frequency bands, with 53 (234) detected at 100 (150) GHz alone; 170 sources are identified as ultracompact $\mathrm{H}$ II regions. Approximating the distribution of total intensity source fluxes as a power law, we find a slope of $\gamma_{S, 100}=-1.8 \pm 0.4$ at $100 \mathrm{GHz}$ and $\gamma_{S, 150}=-2.2 \pm 0.4$ at $150 \mathrm{GHz}$. Similarly, the power-law index of the source two-point angular correlation function is $\gamma_{\theta, 100}=-1.21 \pm 0.04$ and $\gamma_{\theta, 150}=$ $-1.25 \pm 0.04$. The total intensity spectral index distribution peaks at $\alpha_{I} \sim 0.25$, indicating that dust emission is not the only source of radiation produced by these objects between 100 and $150 \mathrm{GHz}$; free-free radiation is likely significant in the $100 \mathrm{GHz}$ band. Four sources are detected in polarized intensity $P$, of which three have matching counterparts in $I$. Three of the polarized sources lie close to the Galactic center, Sagittarius A*, Sagittarius B2, and the Galactic Radio Arc, while the fourth is RCW 49, a bright H II region. An extended polarized source, undetected by the source extraction algorithm on account of its $\sim 0.5$ size, is identified visually, and is an isolated example of large-scale polarized emission oriented distinctly from the bulk Galactic dust polarization.
\end{abstract}

Key words: Galaxy: center - Galaxy: structure - H II regions - radio continuum: ISM - stars: formation - surveys

Online-only material: color figures, machine-readable table

\section{INTRODUCTION}

Millimeter ( $\mathrm{mm}$ ), submillimeter (sub-mm), and far-infrared (FIR) observations are ideal for studying the properties of starforming regions in the galaxy, in particular the cool envelopes of dust and gas that host sites of potential and active star formation. By spanning the peak in the spectra of these objects, measurements between the mm and FIR can tightly constrain the parameters of the thermal radiation produced by the dust. In particular, the mass of a star-forming core and its surrounding envelope is well traced by its measured flux in these bands, since this radiation is optically thin at sub-mm and longer wavelengths.

\footnotetext{
${ }^{14}$ Current address: Owens Valley Radio Observatory, Big Pine, CA 93513, USA.

${ }^{15}$ Current address: School of Physics and Astronomy, University of Manchester, Manchester M13 9PL, UK.

${ }^{16}$ Current address: Infrared Processing and Analysis Center, California Institute of Technology, Pasadena, CA 91125, USA.
}

Surveys covering large sections of the galaxy have the potential to collect statistical samples of cores in a range of evolutionary states comparatively free of bias introduced by targeting particular regions. These surveys are ideal for studying processes related to star-forming regions, such as measuring the core mass function (from which the stellar initial mass function (IMF) may be derived), particularly at the high-mass end, which, on account of the short-lived high mass cores, is understudied relative to lower masses (e.g., Enoch et al. 2006, 2008; Young et al. 2006). Combination with infrared (IR) data yields insight into the ages of cores, permitting differentiation between prestellar sub-mm cores, which lack an IR counterpart, and protostellar cores, in which the ultraviolet radiation produced by protostars is re-radiated into the $\mathrm{mm}$, sub-mm, and IR by the surrounding envelope. Phenomena associated with later evolutionary phases, such as mass ejection, dissipation of the envelope, and dynamical interactions are not significant in the prestellar or protostellar stage-the mass and spatial distribution of such cores therefore capture information regarding the fragmentation process (Enoch et al. 2006). 
Observations of polarized radiation permit a window through which to study the role of magnetic fields (e.g., Greaves et al. 1995; Novak et al. 1997) and their role in providing support against collapse. In the $\mathrm{mm}$ and sub-mm, polarization is due to emission along the long axis of dust grains partially aligned by the magnetic field, and thus measurements of the dust polarization directly probe local magnetic fields (e.g., Hildebrand 1988). These fields are thought to strongly influence the evolution of molecular clouds, since they provide support preventing the collapse of the gas and subsequent triggering of star formation.

Several large-scale surveys are already underway or completed to help address these questions. Herschel (Pilbratt et al. 2010) and Planck (Villa et al. 2002) will provide extensive spectral coverage from the radio to the FIR, fully characterizing the spectral energy distribution (SED) of star-forming cores over the full sky; selected existing results in targeted regions include, e.g., Hennemann et al. (2010), Juvela et al. (2010), and André et al. (2010), but are limited to total intensity observations. Ground and balloon instruments also contribute substantially to the literature: Schuller et al. (2009) present an APEX LABOCA $95 \mathrm{deg}^{2}$ survey in total intensity with resolution of $19^{\prime \prime} .2$ at $353 \mathrm{GHz}$, with the final survey coverage expected to reach $350 \mathrm{deg}^{2}$; Bolocam has mapped $150 \mathrm{deg}^{2}$ of the first Galactic quadrant at $1.1 \mathrm{~mm}(268 \mathrm{GHz})$ with resolution $33^{\prime \prime}$, with a source catalog presented in Rosolowsky et al. (2010); BLAST (Olmi et al. 2009; Netterfield et al. 2009) provides a $50 \mathrm{deg}^{2}$ survey of the Vela molecular cloud at 250, 350, and $500 \mu \mathrm{m}(36,42$, and 60 arcsec resolution, respectively).

Observations at comparable resolution are currently scarce at $\sim 100 \mathrm{GHz}$, and yet provide additional constraining power to the Rayleigh-Jeans tail of the thermal dust spectrum, and probe for contributions due to other emission mechanisms which contribute increasingly at lower frequencies (e.g., free-free). Furthermore, there is little high angular resolution polarization data at these frequencies, despite their utility in understanding star-forming regions.

In this paper, we present a catalog of compact sources found in the QUaD Galactic Plane Survey (Culverhouse et al. 2010), which covers over $\sim 800 \mathrm{deg}^{2}$ of the low-latitude Galactic plane at 100 and $150 \mathrm{GHz}$ with beam FWHM of $5^{\prime}$ and 3'.5, respectively, in Stokes $I, Q$, and $U$ parameters. ${ }^{17}$ A survey of this size, frequency, and angular resolution can be used to investigate the polarized and unpolarized properties of both diffuse emission and discrete sources. The QUaD survey was conducted blind, in that no region was specifically targeted. In principle, this allows the construction of statistical samples of cores, representative of the distribution of core masses and ages in the Galaxy as a whole. However, we note that at the $\sim$ few arcminute resolution of the survey, the maps do not generally resolve individual cores. Dense cores typically have a size of $\sim 0.1$ pc (e.g., Williams et al. 2000), hence for nominal distances of a few hundred parsecs, the sources presented here should be considered "clumps" hosting cores rather than individual cores themselves. In addition to the lack of resolution and accurate clump distances, the contribution of free-free emission at $100 \mathrm{GHz}$ biases measurements of the flux from the dust component; these caveats prevent reliable mass calculation. Our goals here are therefore to analyze the observed quantities of the sources in the survey, rather than infer their physical properties.

\footnotetext{
17 The QUaD maps and source catalogs analyzed in this paper are available
} for public download at http://find.spa.umn.edu:/quad/quad_galactic/.
Basic information on the instrument, observations, and maps is presented in Section 2. In Section 3, we describe our algorithm for extracting sources in the presence of a diffuse background. The global properties of the catalog are discussed in Section 4, with the full catalogs presented in Table 2 (total intensity) and Table 3 (polarized intensity). Discussion and Conclusions are found in Section 5. Extensive simulations, presented in the Appendix, are used to quantify the effects of mapmaking and the source extraction algorithm on the recovered source properties.

\section{INSTRUMENT, OBSERVATIONS, AND MAPS}

Here, we summarize the features of the QUaD Galactic Plane Survey. A detailed description of the instrument can be found in Hinderks et al. (2009), hereafter referred to as the "Instrument Paper." The field selection, survey strategy, data processing, and construction of the Stokes $I, Q$, and $U$ maps are presented in Culverhouse et al. (2010), hereafter the "Map Paper."

QUaD was a $2.6 \mathrm{~m}$ Cassegrain radio telescope on the mount originally constructed for the DASI experiment (Leitch et al. 2002). This is an az/el mount, with a third axis allowing the entire optics and receiver to be rotated around the line of sight. The mount is enclosed in a reflective ground shield, extended from DASI, on top of a tower at the MAPO observatory approximately $1 \mathrm{~km}$ from the geographic South Pole.

The QUaD receiver consisted of 31 pairs of polarization sensitive bolometers (PSBs; Jones et al. 2003), 12 at $100 \mathrm{GHz}$ and 19 at $150 \mathrm{GHz}$. The bolometers were read out using $\mathrm{AC}$ bias electronics and digitized by a $100 \mathrm{~Hz}, 16$ bit ADC; the raw data were staged on disk at Pole and transferred out daily via satellite.

The observations reported in this paper were made between 2007 July and October, with the telescope decommissioned in late 2007. In total QUaD surveyed the Galaxy for 40 days, covering a total of $\sim 800 \mathrm{deg}^{2}$. The survey is divided into two regions, approximately covering $245^{\circ}-295^{\circ}$ and $315^{\circ}-5^{\circ}$ in Galactic longitude $l$, and $-4^{\circ}$ to $+4^{\circ}$ in Galactic latitude $b$. These regions are loosely called the "third quadrant" and "fourth quadrant" throughout.

Maps are made by co-adding the timestream from each detector into flat-sky (R.A., decl.) pixels of size $0.02 \times 0.02$. The absolute pointing accuracy was determined to be $\sim 0.5 \mathrm{rms}$, using pointing checks on RCW 38 and other Galactic sources over two seasons of cosmic microwave background (CMB) observations (see the Instrument Paper for further details). A field-differencing scheme was used to remove spurious ground contamination. Data acquisition was divided into $2 \mathrm{hr}$ blocks. In the first, the telescope scanned over a "lead" field centered on the plane of the Galaxy. The next hour of data covered a "trail" field offset $1 \mathrm{hr}$ later in R.A., such that the azimuth and elevation range scanned by the telescope with respect to the ground was identical for both the lead and trail fields. By differencing the lead and trail field timestream, any spurious ground pickup is removed. The results presented here are derived using fielddifferenced maps; the consequences of field-differencing in the context of the source catalog are discussed further in Section 3.1, while we refer the reader to the Map Paper for a more detailed discussion of field-differencing. Absolute calibration is applied using a scaling factor at each frequency, derived in the QUaD CMB analysis presented in Brown et al. (2009). These factors were calculated by cross-calibrating QUaD CMB maps to those from the Boomerang experiment (Masi et al. 2006) and have an estimated uncertainty of $3.5 \%$. The $100 \mathrm{GHz} I$ map for both survey regions is shown in Figure 1; the reader is referred to 

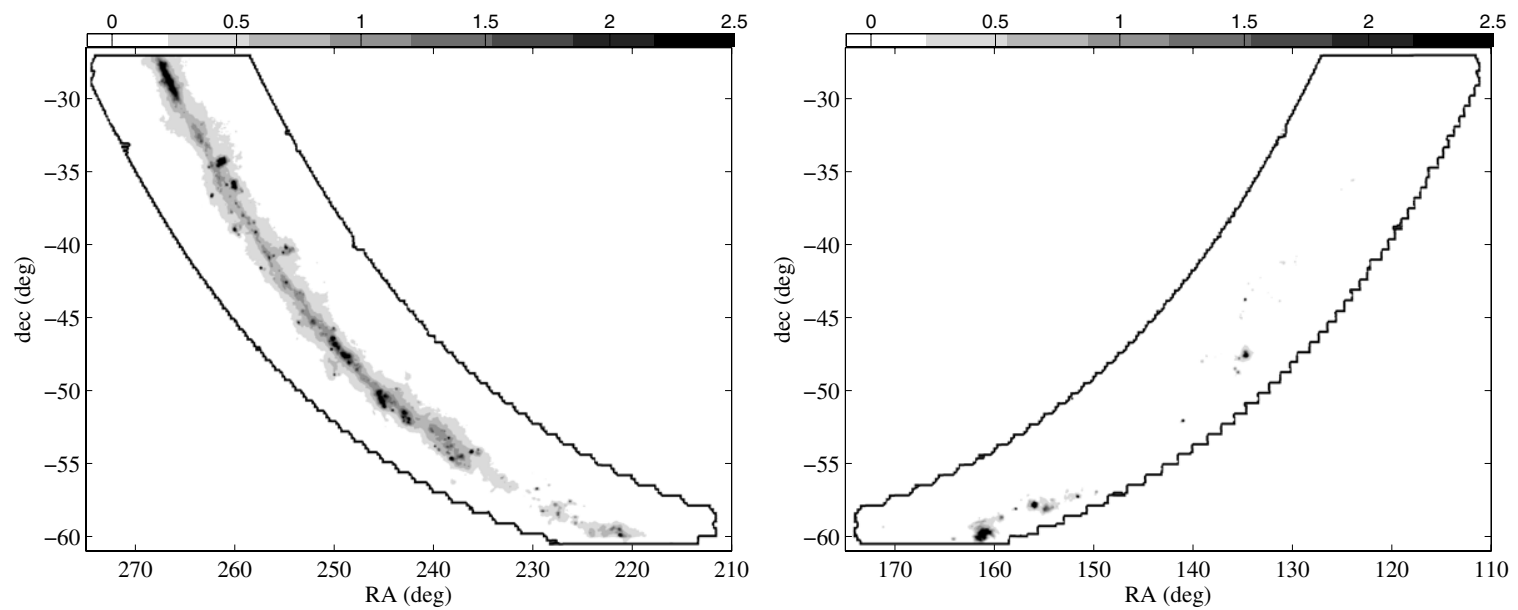

Figure 1. Fourth (left) and third (right) quadrant field-differenced $100 \mathrm{GHz}$ Stokes $I$ map smoothed to the beam scale $\left(5^{\prime}\right)$, with color scale in MJy sr ${ }^{-1}$. The solid black lines indicate the survey coverage.

the Map Paper for similar maps at both frequencies and in $I$, $Q$, and $U$. In addition to the sky maps, variance maps for each Stokes parameter are also constructed, which give a measure of the noise in each map pixel. The typical survey sensitivity in each survey area is 74 (107) $\mathrm{kJy} \mathrm{sr}^{-1}$ at 100 (150) $\mathrm{GHz}$ in $I$, and $98(120) \mathrm{kJy} \mathrm{sr}^{-1}$ in $Q / U$, at a spatial resolution of 5 (3.5) arcmin at $100(150) \mathrm{GHz}$. The orientation of $Q$ and $U$ in the QUaD polarization maps follows the IAU convention (Hamaker \& Bregman 1996), in which $+Q$ is parallel to $\mathrm{N}-\mathrm{S}$ and $+U$ parallel to NE-SW.

\section{SOURCE EXTRACTION}

As is readily apparent from Figure 1, a substantial contributor to the sky signal is diffuse emission. This "background" increases the uncertainty in measured properties of compact sources above that due to detector and atmospheric noise. However, the systematic effect of diffuse emission can be reduced using spatial filtering.

The source extraction method implemented here is an adaptation of the algorithm described in Désert et al. (2008), a Mexican-hat linear filter in image space, which was designed to separate compact sources from the diffuse Galactic emission for the Archeops experiment. Maps of each stage of the source extraction algorithm, described below, are shown in Figure 2 in a representative section of the fourth quadrant $100 \mathrm{GHz} I$ data.

In our algorithm, two sets of smoothed $I$ and $P=\sqrt{Q^{2}+U^{2}}$ maps are made at each frequency; both are derived from the raw maps $m_{r}$. The first set, $m_{b}$, consists of $m_{r}$ smoothed to the beam scale $\sigma_{\text {beam }}$. In the second, $m_{r}$ is smoothed to an angular scale $\sigma_{\text {bck }}=2.5 \times \sigma_{\text {beam }}$ to form a template map of the diffuse background, $m_{\text {bck }}$. In both cases, a circularly symmetric Gaussian function is the smoothing kernel. The choice of $\sigma_{\text {bck }}$ is designed to minimize the background contribution to source fluxes without introducing large biases in the measured flux. In Appendix A.5, simulations demonstrate the consequences of other choices of $\sigma_{\text {bck }}$ on recovered source fluxes. The background maps are then subtracted from beam-smoothed maps to yield the "source extraction" map $m_{s}$ :

$$
m_{I, s}=m_{I, b}-m_{I, \mathrm{bck}}
$$

and likewise for $P$. Note that since $\sigma_{\text {bck }}>\sigma_{\text {beam }}$, Equation (1) is equivalent to convolving $m_{r}$ with filter constructed from the difference of the two smoothing kernels, commonly referred to as a "Mexican hat" filter.

Negative pixels due to ringing are masked, and the remaining pixels are subjected to signal-to-noise thresholding. Pixels above a signal-to-noise $(\mathrm{S} / \mathrm{N})$ threshold of 5 (3) for total (polarized) intensity are flagged as belonging to source candidates. The polarization data have a lower extraction threshold because the noise properties are closer to white on account of the unpolarized atmosphere, and also because the diffuse component amplitude (fractional polarization $<2 \%$; see Map Paper) is close to the instrumental noise level and therefore its effect on source fluxes is small. High signal-to-noise regions in the $P$ map define a set of pixels to which we separately fit polarized sources in the $Q$ and $U$ maps. Candidate pixels in all Stokes maps are subject to suitability checks; isolated pixels or groups of pixels smaller than the beam width are removed.

Sources in close proximity tend to be members of the same thresholded region, so an internally developed segmentation algorithm based on the SExtractor code (Bertin \& Arnouts 1996) is used to split such regions into separate sources. Source segmentation is applied to the $I, Q$, and $U$ maps separately at each frequency, resulting in a set of six source position lists, along with the map pixels assigned to each source.

Having determined source positions, the background maps $m_{\text {bck }}$ are regenerated by again smoothing $m_{r}$, but with the pixels corresponding to discrete sources replaced by the local median-this "source-corrected" background map is denoted $m_{\text {bck,corr }}$, with $m_{s, \text { corr }}=m_{b}-m_{\text {bck,corr }}$ following from Equation (1). The median-replacement step reduces the amount of ringing due to the background filtering implemented in Equation (1) (see Figure 2). The resulting background map contains less leaked flux due to smoothing discrete sources with a kernel larger than the beam size. The background subtraction, source detection, and segmentation stages are then repeated.

All ingredients for measuring source properties are present at this point: the background map $m_{\mathrm{bck}, \mathrm{corr}}$, a list of pixels belonging to each source, and the input map itself $m_{r}$, with its variance map $\sigma_{r}^{2}$. The background map $m_{\text {bck,corr }}$ is subtracted from the input map $m_{r}$ yielding the map to which source models are fit,

$$
m_{I, f}=m_{I, r}-m_{I, \mathrm{bck}, \mathrm{corr}},
$$

and likewise for $P$. An elliptical Gaussian is fit to the resulting pixels for each source; an example of the model reconstructed from these fits is shown in Figure 2. Residuals of this model 

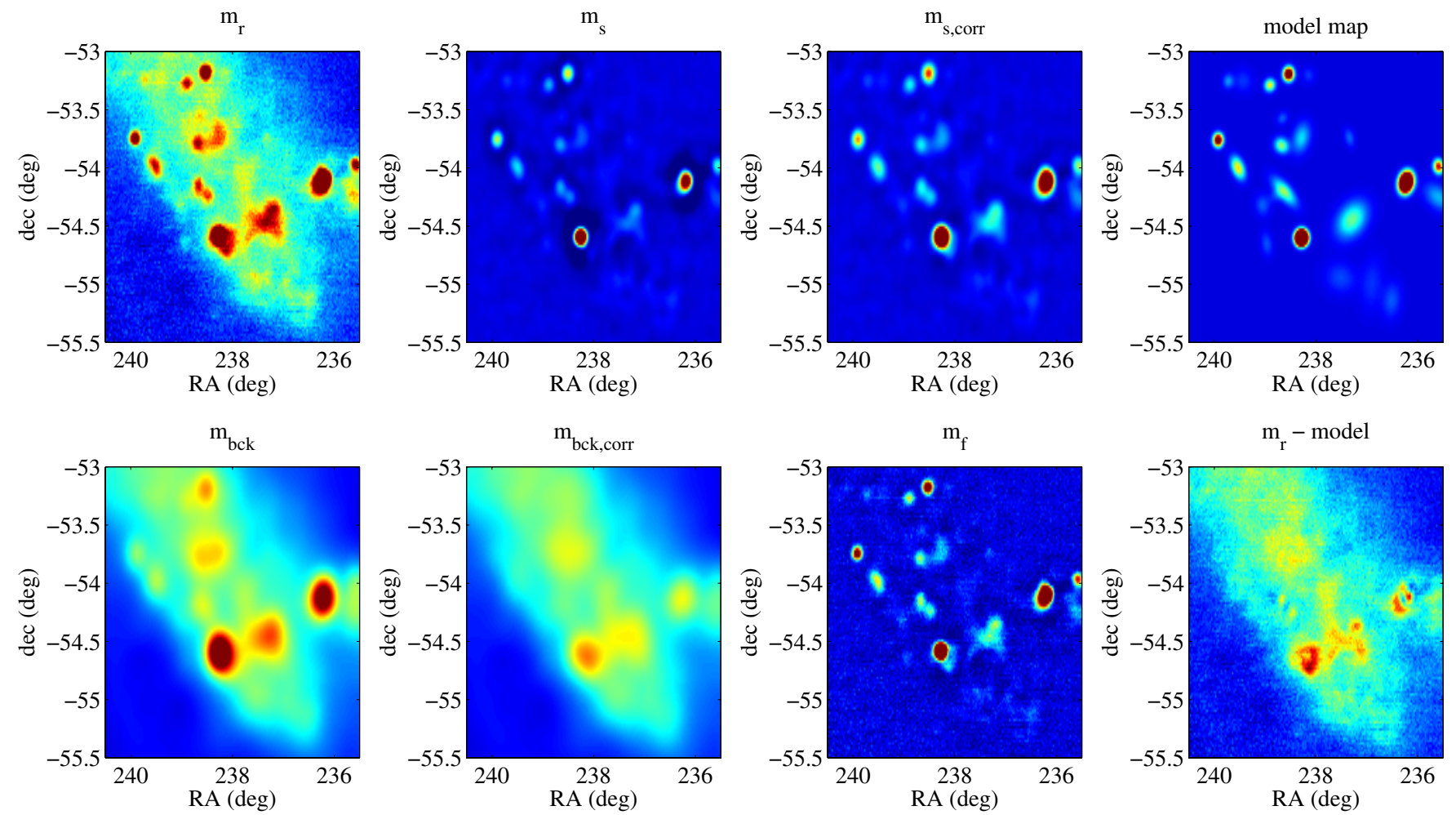

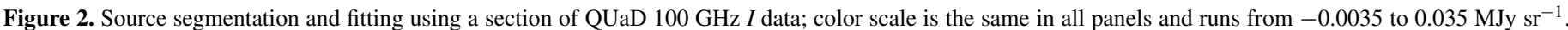

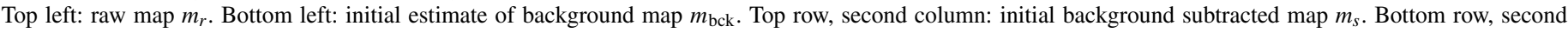

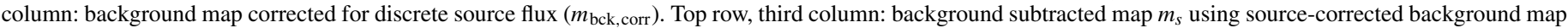

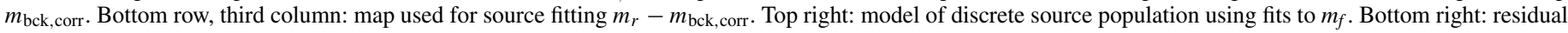
between model sky and input image $m_{r}$.

(A color version of this figure is available in the online journal.)

against $m_{r}$, also shown in Figure 2, indicate that the sourcefitting works well, except for two cases: (1) particularly bright sources, which can leave residuals at the $\sim$ few percent level, and (2) sources in close proximity, for which the source segmentation fails and the sources are classed as a single object.

Fits are performed independently at each frequency in $I$, $Q$, and $U$, with pixel noise taken from the corresponding variance map $\sigma_{r}^{2}$; errors on source properties follow directly from the parameter errors returned by the fit minimizer. The elliptical Gaussian fit parameters are used to calculate source properties such as flux and position; in a small number of cases, the uncertainty on a fit parameter diverges, in which case we do not quote the uncertainty on any physical quantity derived from this parameter. Derived quantities such as spectral index $\alpha=\log \left(I_{2} / I_{1}\right) / \log \left(v_{2} / \nu_{1}\right)$, polarization angle $\phi=$ $0.5 \tan ^{-1}(U / Q)$, polarization fraction $P / I$, and their associated errors are calculated from $I, Q$, and $U$ fluxes. In general, a source detected in $I$ will not have the same set of pixels as in $Q$ or $U$ as each map is treated independently; sources are spatially matched across catalogs later to determine, e.g., polarization fraction. Given two catalogs A and B (such as total intensity at two frequencies), each source in A is matched to a source in B if their angular separation is less than three map pixels (3'6), conservatively larger than the rms day-to-day telescope pointing wander of $\sim 0$.'5. If more than one source in $B$ matches a source in A, as can happen when matching sources between 100 and $150 \mathrm{GHz}$ due to the higher resolution in the latter band, the closer of the two is selected. Having matched sources, the corresponding physical quantities are combined to yield the derived quantity such as the spectral index.

\subsection{Consequences of Field Differencing}

The field-differencing operation performed on the timestream to remove ground contamination can result in spurious sources in the data. If a source lies in the trail field of the observations (at larger R.A.), when the trail field is differenced against the lead field the source will appear negative in the lead field, resulting in a negative measured flux. Such sources are removed in $I$ by rejecting candidates with fluxes below zero at a signal-to-noise of 5 or greater. This rejection is not possible in $Q$ or $U$ because the polarized flux can take positive or negative values. Instead, detected polarized sources are matched to the total intensity source catalog; if the polarized source is matched to a source of negative total intensity with $|\mathrm{S} / \mathrm{N}|>5$, the polarized source is removed from the catalog. This method may allow small numbers of field differenced polarized sources to leak into the catalog, since sources in $I$ are extracted at a higher significance threshold $(5 \sigma)$ than polarization - a field differenced source in $P$ may not be matched to a total intensity candidate and therefore cannot be rejected.

Field-differenced sources are also increasingly expected at low declinations. The central PSB pair are aligned on the plane of the Galaxy, with the low R.A. edge of the trail field aligned with the high R.A. edge of the lead field; the width of the QUaD focal plane allows for overlapping coverage of the lead and trail fields. At higher elevation (lower declination) the scan throw of $\delta \mathrm{Az}=15^{\circ}$ translates into a smaller R.A. range as $\delta$ R.A. $=\delta \mathrm{Az} \times \cos (\mathrm{decl}$.). Due to the alignment of lead and trail field edges and the decreasing scan throw in R.A. at lower decl., the trail field lies closer to the galactic plane at low decl.- see 

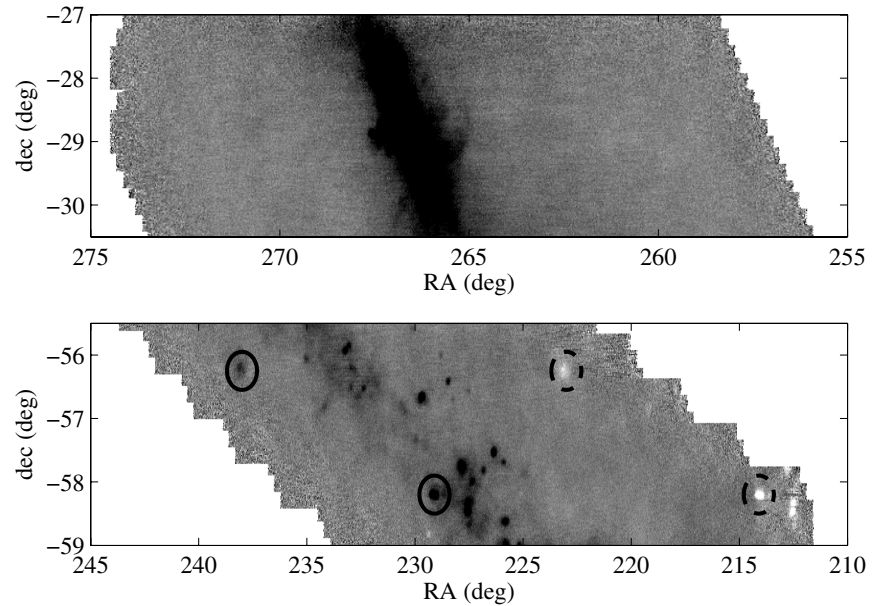

Figure 3. Top: subsection of field-differenced $100 \mathrm{GHz} I$ map covering galactic center. Few sources lie near the left (high R.A.) edge of the map, where the trail field is aligned. Bottom: subsection of field-differenced $100 \mathrm{GHz} I$ map covering the lowest decl. of the survey. Two real sources close to the trail field region (high R.A. edge) are circled with solid black lines. Their field-differenced counterparts are circled with broken black lines; notice the change in sign in intensity of these spurious sources (other field-differenced sources are also visible). No sources lie as close to the trail field edge in the top plot, and hence no spurious sources are detected at this decl.

Figure 3 (we further refer the reader to Figure 2 of the Map Paper for a graphical representation of the lead/trail field geometry over the full survey). At lower decl. the trail field is therefore more likely to contain a bright source (Figure 3 ). However, the QUaD catalog indicates that most detected sources lie within $3^{\circ}$ of the Galactic plane (Section 4.2), and thus the contribution of field differenced sources is small over most of the survey, since the trail fields never get closer than $\sim\left(15^{\circ} / 2\right) \times \cos (60)=3.75$ to $b=0$.

\section{RESULTS}

Source catalogs from both the third and fourth quadrant maps are extracted and combined for the purposes of calculating statistical properties. Spurious sources are rejected if the flux is negative at one frequency and undetected at the other frequency. Statistics for the survey are shown in Table 1.

In total, 292 (473) sources are detected in $I$ at $100(150) \mathrm{GHz}$, of which 239 are spatially matched between frequency bands, resulting in 526 unique sources in total intensity. Position, major/minor axes, flux, and spectral index for each source in $I$ are given in Table 2. Four sources are detected in $P$, of which two are matched spatially across bands; three of these polarized sources have matching counterparts in at least one frequency band in $I$. Properties of these sources are presented in Table 3.

Simulations are used to determine survey completeness and purity and accuracy of recovered source parameters. Four different types of simulations (labeled Sim1-Sim4) are used to calculate these quantities and how they are affected by features particular to a Galactic plane survey, specifically the influence of the diffuse background, and the effect of an anisotropic distribution of spatially clustered point sources. A detailed description of the simulations is presented in the Appendix.

The $90 \%$ survey completeness in total intensity, $C_{I, 90}$, is determined from Sim4 (the most realistic simulation used, incorporating both detector, atmospheric noise, spatially clustered point sources, and a model for the diffuse background); we find 5.2 and $2.8 \mathrm{Jy}$ at 100 and $150 \mathrm{GHz}$, respectively. These do not
Table 1

Source Statistics

\begin{tabular}{lccc}
\hline \hline Type & $100 \mathrm{GHz}$ & $150 \mathrm{GHz}$ & Freq Matched \\
\hline$I$ & 292 & 473 & 239 \\
$P$ & 3 & 3 & 2 \\
$I / P$ matched & 2 & 2 & 1 \\
\hline
\end{tabular}

change significantly with the inclusion/exclusion of a diffuse background, indicating that the background removal strategy described in Section 3 is effective.

In polarization, the $90 \%$ completeness $C_{P, 90}=1.2 \mathrm{Jy}$ at $100 \mathrm{GHz}$ and $0.9 \mathrm{Jy}$ at $150 \mathrm{GHz}$. Similar to total intensity, the completeness limit in polarization is not strongly dependent on the presence of diffuse Galactic emission.

At the signal-to-noise extraction threshold of 5 in $I$, the survey is $90 \%-100 \%$ pure at both frequencies, while the purity in polarization is very similar, but at an extraction signal-to-noise of 3 ; the spread in purity arises due to the different types of simulation.

The QUaD catalog is matched to the IRAS Point Source Catalog (IRAS-PSC) and the Parkes-MIT-NRAO (PMN) catalog (Condon et al. 1993) using a search radius of 3.6-the same as used for matching sources between the two QUaD bands. We find $80 \%(55 \%)$ of sources at 100 (150) GHz have IRAS counterparts, while three sources without IRAS-PSC counterpart have associations with PMN sources. The adopted search radius accounts for the likely range of potential offsets between sources detected by QUaD and IRAS due to pointing wander $(\sim 0.5 \mathrm{rms}$ for $\mathrm{QUaD}$ ), and systematic offsets due to different background filtering methods (which could bias source centroids due to differing amounts of unfiltered background substructure). A smaller radius could fail to match sources based on these considerations, while a larger radius could introduce false matches due to chance association; increasing the search range to $6^{\prime}$ results in $97 \%(87 \%)$ QUaD sources matched to IRAS at $100(150) \mathrm{GHz}$. False matching is particularly problematic at low flux density, where simulations indicate that resolved diffuse emission can often be misidentified as discrete sources (Appendix A.3). The adopted search radius of 3.6 is therefore a compromise between these effects.

The sources with IR counterparts indicate that cores located inside these detected clumps are past the prestellar phase and have thermally radiating dust envelopes. This result might be expected; since the QUaD frequency bands probe the dust emission well away from the core SED peak, we are unlikely to detect the prestellar or starless cores which consist solely of very cold molecular gas and have no internal source of luminosity. Sources detected at $150 \mathrm{GHz}$ without IRAS counterparts may be due to a single IRAS source being resolved into multiple components, or objects whose emission is dominated by synchrotron or free-free, and have IR emission below the IRAS flux limit. The sensitivity of QUaD to free-free emission (particularly at $100 \mathrm{GHz}$ ) makes the survey more likely to detect clumps in an advanced evolutionary phase where an $\mathrm{H}$ II region has already formed, rather than a cold starless core whose emission consists only of thermal dust.

The QUaD catalog can provide constraints on the continuum spectra of each source. However, source fluxes at $100 \mathrm{GHz}$ should be interpreted carefully due to the possible contribution of free-free emission at this frequency. For QUaD sources with an IRAS counterpart, IRAS FIR fluxes can be used to identify ultracompact H II (UCH II) regions using the Wood-Churchwell 
Table 2

QUaD Galactic Source Catalog

\begin{tabular}{|c|c|c|c|c|c|c|c|c|c|c|c|c|c|c|}
\hline \multirow[t]{2}{*}{ Source } & \multirow[b]{2}{*}{$\begin{array}{l}\text { R.A. } \\
\text { (deg) }\end{array}$} & \multirow[b]{2}{*}{$\begin{array}{l}\text { Decl. } \\
\text { (deg) }\end{array}$} & \multicolumn{4}{|c|}{$100 \mathrm{GHz}$} & \multicolumn{4}{|c|}{$150 \mathrm{GHz}$} & \multirow[t]{2}{*}{$\alpha_{I}$} & \multirow[t]{2}{*}{$\delta \alpha_{I}$} & \multirow[t]{2}{*}{ Alt. Name } & \multirow[t]{2}{*}{$\mathrm{WC}$} \\
\hline & & & $\begin{array}{c}\sigma_{\text {maj }} \\
(\operatorname{arcmin})\end{array}$ & $\begin{array}{c}\sigma_{\min } \\
(\operatorname{arcmin})\end{array}$ & $\begin{array}{c}I \\
(\mathrm{Jy})\end{array}$ & $\begin{array}{c}\delta I \\
(\mathrm{Jy})\end{array}$ & $\begin{array}{c}\sigma_{\text {maj }} \\
(\operatorname{arcmin})\end{array}$ & $\begin{array}{c}\sigma_{\min } \\
(\operatorname{arcmin})\end{array}$ & $\begin{array}{c}I \\
(\mathrm{Jy})\end{array}$ & $\begin{array}{c}\delta I \\
(\mathrm{Jy})\end{array}$ & & & & \\
\hline $253.61-0.18$ & 124.08 & -35.56 & 21.7 & 3.5 & 9.38 & 7.86 & 3.8 & 1.8 & 1.75 & 11.19 & -3.66 & 14.04 & IRAS 08143-3521 & 0 \\
\hline $254.69+0.21$ & 125.23 & -36.23 & 2.1 & 1.9 & 2.08 & 0.13 & 1.5 & 1.4 & 2.55 & 0.16 & 0.45 & 0.19 & IRAS 08189-3602 & 1 \\
\hline $261.65-2.08$ & 128.06 & -43.23 & $\ldots$ & $\ldots$ & $\ldots$ & $\ldots$ & 1.8 & 1.4 & 0.96 & 0.18 & $\ldots$ & $\ldots$ & IRAS 08286-4251 & 0 \\
\hline $262.11-1.80$ & 128.74 & -43.43 & $\ldots$ & $\ldots$ & $\ldots$ & $\ldots$ & 5.1 & 2.1 & 2.59 & 0.90 & $\ldots$ & $\ldots$ & none & 0 \\
\hline $259.95-0.03$ & 128.90 & -40.64 & $\ldots$ & $\ldots$ & $\ldots$ & $\ldots$ & 2.0 & 1.7 & 1.22 & 0.32 & $\ldots$ & $\ldots$ & IRAS 08318-4020 & 0 \\
\hline $260.81+0.68$ & 130.33 & -40.89 & $\ldots$ & $\ldots$ & $\ldots$ & $\ldots$ & 2.0 & 1.1 & 0.78 & 0.24 & $\ldots$ & $\ldots$ & none & 0 \\
\hline $263.63-0.53$ & 131.41 & -43.86 & 2.6 & 2.3 & 2.19 & 0.15 & 2.1 & 1.7 & 4.08 & 0.16 & 1.35 & 0.17 & IRAS 08438-4340 & 1 \\
\hline $263.79-0.43$ & 131.65 & -43.92 & $\ldots$ & $\ldots$ & $\ldots$ & $\ldots$ & 1.6 & 1.5 & 1.55 & 0.14 & $\ldots$ & $\ldots$ & IRAS 08432-4335 & 0 \\
\hline $263.26+0.52$ & 132.21 & -42.91 & $\ldots$ & $\ldots$ & $\ldots$ & $\ldots$ & 1.8 & 1.6 & 1.60 & 0.17 & $\ldots$ & $\ldots$ & PMN J0848-4253 & 0 \\
\hline $263.74+0.16$ & 132.24 & -43.51 & $\ldots$ & $\ldots$ & $\ldots$ & $\ldots$ & 12.3 & 3.9 & 9.60 & 16.47 & $\ldots$ & $\ldots$ & IRAS 08454-4307 & 0 \\
\hline $262.26+1.45$ & 132.34 & -41.55 & $\ldots$ & $\ldots$ & $\ldots$ & $\ldots$ & 3.0 & 1.9 & 2.09 & 0.36 & $\ldots$ & $\ldots$ & none & 0 \\
\hline $264.35-0.19$ & 132.41 & -44.20 & $\ldots$ & $\ldots$ & $\ldots$ & $\ldots$ & 2.3 & 1.6 & 1.49 & 0.21 & $\ldots$ & $\ldots$ & none & 0 \\
\hline $264.69-0.29$ & 132.60 & -44.53 & $\ldots$ & $\ldots$ & $\ldots$ & $\ldots$ & 2.3 & 1.7 & 1.82 & 0.20 & $\ldots$ & $\ldots$ & none & 0 \\
\hline $263.30+1.58$ & 133.35 & -42.27 & 4.9 & 2.9 & 1.58 & 0.56 & 4.3 & 1.8 & 4.43 & 0.32 & 2.25 & 0.79 & IRAS 08516-4204 & 1 \\
\hline $264.31+1.47$ & 134.13 & -43.11 & 2.6 & 2.3 & 2.60 & 0.15 & 1.8 & 1.7 & 2.77 & 0.16 & 0.14 & 0.18 & IRAS 08546-4254 & 1 \\
\hline $264.29+1.74$ & 134.40 & -42.92 & $\ldots$ & $\ldots$ & $\ldots$ & $\ldots$ & 2.5 & 1.7 & 1.68 & 0.23 & $\ldots$ & $\ldots$ & none & 0 \\
\hline $264.16+1.99$ & 134.54 & -42.66 & 5.2 & 2.8 & 1.87 & 0.62 & 2.9 & 2.6 & 4.44 & 0.30 & 1.88 & 0.74 & IRAS 08563-4225 & 0 \\
\hline $264.31+1.99$ & 134.67 & -42.78 & $\ldots$ & $\ldots$ & $\ldots$ & $\ldots$ & 6.0 & 3.2 & 5.50 & 1.12 & $\ldots$ & $\ldots$ & IRAS 08549-4223 & 0 \\
\hline $267.96-1.06$ & 134.78 & -47.53 & 2.6 & 2.4 & 123.14 & 0.15 & 1.9 & 1.8 & 112.61 & 0.16 & -0.19 & 0.00 & IRAS 08573-4718 & 0 \\
\hline $265.68+0.98$ & 134.86 & -44.47 & $\ldots$ & $\ldots$ & $\ldots$ & $\ldots$ & 11.7 & 2.6 & 5.84 & 4.65 & $\ldots$ & $\ldots$ & none & 0 \\
\hline $267.84-0.88$ & 134.87 & -47.32 & 4.1 & 3.0 & 7.10 & 0.25 & 4.8 & 3.2 & 9.75 & 0.47 & 0.69 & 0.13 & none & 0 \\
\hline $265.16+1.45$ & 134.88 & -43.76 & 2.6 & 2.5 & 17.64 & 0.15 & 2.1 & 1.9 & 20.68 & 0.17 & 0.35 & 0.03 & IRAS 08576-4334 & 1 \\
\hline $264.98+1.61$ & 134.88 & -43.53 & $\ldots$ & $\ldots$ & $\ldots$ & $\ldots$ & 3.0 & 2.1 & 2.28 & 0.33 & $\ldots$ & $\ldots$ & none & 0 \\
\hline $264.75+1.85$ & 134.92 & -43.20 & $\ldots$ & $\ldots$ & $\ldots$ & $\ldots$ & 2.1 & 1.8 & 1.11 & 0.34 & $\ldots$ & $\ldots$ & none & 0 \\
\hline $265.30+1.42$ & 134.97 & -43.89 & $\ldots$ & $\ldots$ & $\ldots$ & $\ldots$ & 26.0 & 6.4 & 28.19 & 31.17 & $\ldots$ & $\ldots$ & none & 0 \\
\hline $265.86+1.09$ & 135.13 & -44.53 & $\ldots$ & $\ldots$ & $\ldots$ & $\ldots$ & 2.0 & 1.6 & 1.67 & 0.19 & $\ldots$ & $\ldots$ & none & 0 \\
\hline $265.62+1.38$ & 135.23 & -44.16 & $\ldots$ & $\ldots$ & $\ldots$ & $\ldots$ & 4.6 & 3.1 & 2.91 & 0.93 & $\ldots$ & $\ldots$ & none & 0 \\
\hline $265.85+1.26$ & 135.30 & -44.41 & $\ldots$ & $\ldots$ & $\ldots$ & $\ldots$ & 5.8 & 2.0 & 2.54 & 3.35 & $\ldots$ & $\ldots$ & IRAS $08578-4400$ & 0 \\
\hline $266.27+0.92$ & 135.34 & -44.95 & $\ldots$ & $\ldots$ & $\ldots$ & $\ldots$ & 4.3 & 2.9 & 2.92 & 1.31 & $\ldots$ & $\ldots$ & none & 0 \\
\hline $268.44-0.85$ & 135.49 & -47.75 & 2.4 & 2.2 & 7.06 & 0.13 & 1.9 & 1.6 & 11.24 & 0.15 & 1.01 & 0.05 & IRAS 09002-4732 & 1 \\
\hline $269.20-1.43$ & 135.59 & -48.70 & 3.3 & 3.1 & 10.93 & 0.19 & 3.1 & 2.6 & 10.72 & 0.29 & -0.04 & 0.07 & IRAS 09006-4830 & 1 \\
\hline $268.42-0.49$ & 135.87 & -47.50 & $\ldots$ & $\ldots$ & $\ldots$ & $\ldots$ & 2.1 & 1.5 & 0.94 & 0.22 & $\ldots$ & $\ldots$ & IRAS 08598-4706 & 0 \\
\hline $269.14-1.12$ & 135.89 & -48.46 & 2.7 & 2.4 & 7.05 & 0.15 & 2.2 & 1.9 & 10.15 & 0.20 & 0.79 & 0.06 & IRAS 09018-4816 & 1 \\
\hline $270.83-1.12$ & 137.63 & -49.70 & $\ldots$ & $\ldots$ & $\ldots$ & $\ldots$ & 2.5 & 1.3 & 0.94 & 0.40 & $\ldots$ & & IRAS 09071-4915 & 0 \\
\hline $270.14-0.28$ & 137.84 & -48.62 & $\ldots$ & $\ldots$ & $\ldots$ & $\ldots$ & 3.6 & 1.4 & 0.97 & 1.06 & $\ldots$ & $\ldots$ & IRAS 09077-4809 & 0 \\
\hline $269.46+0.41$ & 137.89 & -47.65 & $\ldots$ & $\ldots$ & $\ldots$ & $\ldots$ & 2.6 & 1.7 & 1.05 & 0.35 & $\ldots$ & $\ldots$ & none & 0 \\
\hline $269.81+0.84$ & 138.72 & -47.61 & $\ldots$ & $\ldots$ & $\ldots$ & $\ldots$ & 2.0 & 1.8 & 1.92 & 0.19 & $\ldots$ & $\ldots$ & none & 0 \\
\hline $270.29+0.84$ & 139.22 & -47.96 & 3.2 & 2.6 & 3.20 & 0.19 & 2.6 & 1.9 & 3.99 & 0.22 & 0.48 & 0.18 & IRAS 09149-4743 & 1 \\
\hline $270.59+0.83$ & 139.51 & -48.18 & $\ldots$ & $\ldots$ & $\ldots$ & $\ldots$ & 3.5 & 2.3 & 1.84 & 1.13 & $\ldots$ & $\ldots$ & IRAS 09144-4742 & 0 \\
\hline $270.83+0.67$ & 139.60 & -48.47 & $\ldots$ & $\ldots$ & $\ldots$ & $\ldots$ & 3.2 & 2.0 & 2.04 & 0.34 & $\ldots$ & $\ldots$ & IRAS 09150-4805 & 0 \\
\hline $274.02-1.16$ & 141.12 & -52.01 & 3.0 & 2.4 & 24.16 & 0.15 & 2.5 & 1.8 & 25.36 & 0.17 & 0.11 & 0.02 & IRAS 09227-5146 & 1 \\
\hline $278.32-0.93$ & 146.72 & -54.73 & $\ldots$ & $\ldots$ & $\ldots$ & $\ldots$ & 5.9 & 2.8 & 3.76 & 6.36 & $\ldots$ & $\ldots$ & IRAS 09434-5417 & 0 \\
\hline $281.03-1.53$ & 149.81 & -56.89 & 3.5 & 2.9 & 7.45 & 0.33 & 3.3 & 2.2 & 10.12 & 0.39 & 0.67 & 0.13 & IRAS 09575-5640 & 1 \\
\hline $281.18-1.64$ & 149.91 & -57.07 & $\ldots$ & $\ldots$ & $\ldots$ & $\ldots$ & 1.8 & 1.6 & 1.74 & 0.35 & $\ldots$ & $\ldots$ & none & 0 \\
\hline $281.15-1.59$ & 149.92 & -57.01 & 10.4 & 4.0 & 7.91 & 7.60 & $\ldots$ & $\ldots$ & $\ldots$ & $\ldots$ & $\ldots$ & $\ldots$ & IRAS 09578-5649 & 1 \\
\hline $281.61-0.97$ & 151.27 & -56.78 & 3.6 & 2.6 & 1.43 & 0.91 & 2.1 & 1.5 & 1.98 & 0.24 & 0.72 & 1.42 & IRAS 10031-5632 & 1 \\
\hline $282.04-1.18$ & 151.69 & -57.21 & 3.2 & 2.5 & 22.35 & 0.21 & 2.3 & 1.7 & 18.96 & 0.25 & -0.36 & 0.04 & IRAS 10049-5657 & 1 \\
\hline $282.27-1.10$ & 152.12 & -57.28 & 6.4 & 2.0 & 1.34 & 2.20 & $\ldots$ & $\ldots$ & $\ldots$ & $\ldots$ & $\ldots$ & $\ldots$ & IRAS 10067-5705 & 0 \\
\hline $282.24-0.85$ & 152.33 & -57.06 & $\ldots$ & $\ldots$ & $\ldots$ & $\ldots$ & 3.2 & 1.7 & 1.81 & 0.45 & $\ldots$ & $\ldots$ & IRAS 10062-5634 & 0 \\
\hline $282.24-0.47$ & 152.73 & -56.75 & 7.6 & 2.4 & 1.80 & 1.26 & 3.5 & 2.4 & 3.69 & 0.41 & 1.56 & 1.55 & IRAS 10090-5631 & 0 \\
\hline $283.15-0.99$ & 153.57 & -57.70 & 3.4 & 2.2 & 3.09 & 0.22 & 3.0 & 1.8 & 5.02 & 0.32 & 1.06 & 0.21 & IRAS 10123-5727 & 0 \\
\hline $283.36-1.09$ & 153.80 & -57.90 & 5.4 & 4.7 & 5.46 & 1.38 & 3.8 & 2.5 & 3.99 & 0.79 & -0.68 & 0.70 & IRAS 10136-5736 & 0 \\
\hline $283.41-0.86$ & 154.11 & -57.74 & 5.2 & 1.6 & 1.25 & 6.92 & $\ldots$ & $\ldots$ & $\ldots$ & $\ldots$ & $\ldots$ & $\ldots$ & none & 0 \\
\hline $283.56-1.02$ & 154.18 & -57.95 & 5.0 & 3.4 & 5.86 & 0.43 & $\ldots$ & $\ldots$ & $\ldots$ & $\ldots$ & $\ldots$ & $\ldots$ & IRAS 10151-5742 & 0 \\
\hline $283.31-0.57$ & 154.26 & -57.44 & 5.1 & 3.4 & 6.03 & 0.34 & 3.3 & 2.1 & 6.21 & 0.30 & 0.06 & 0.16 & none & 0 \\
\hline $283.95-0.89$ & 154.92 & -58.06 & 10.8 & 7.1 & 33.35 & 0.99 & $\ldots$ & $\ldots$ & $\ldots$ & $\ldots$ & $\ldots$ & $\ldots$ & none & 0 \\
\hline $283.64-0.37$ & 154.98 & -57.45 & 7.4 & 2.7 & 1.97 & 1.68 & $\ldots$ & $\ldots$ & $\ldots$ & $\ldots$ & $\ldots$ & $\ldots$ & none & 0 \\
\hline $284.03-0.87$ & 155.07 & -58.08 & $\ldots$ & $\ldots$ & $\ldots$ & $\ldots$ & 2.8 & 2.4 & 8.52 & 0.34 & $\ldots$ & $\ldots$ & none & 0 \\
\hline $284.31-0.34$ & 156.06 & -57.79 & 4.1 & 3.6 & 143.61 & 0.25 & 3.6 & 3.2 & 138.32 & 0.36 & -0.08 & 0.01 & none & 0 \\
\hline $284.66-0.51$ & 156.44 & -58.12 & $\ldots$ & $\ldots$ & $\ldots$ & $\ldots$ & 3.2 & 2.5 & 2.63 & 0.86 & $\ldots$ & $\ldots$ & none & 0 \\
\hline $284.39-0.02$ & 156.51 & -57.56 & 7.0 & 3.0 & 3.55 & 0.73 & 4.4 & 3.1 & 4.02 & 0.76 & 0.27 & 0.61 & IRAS $10241-5720$ & 0 \\
\hline $284.59-0.19$ & 156.65 & -57.80 & 5.5 & 3.4 & 3.15 & 0.59 & 5.3 & 2.6 & 4.01 & 2.35 & 0.52 & 1.34 & IRAS 10251-5733 & 0 \\
\hline $284.73+0.32$ & 157.37 & -57.45 & 3.2 & 2.0 & 3.07 & 0.16 & 2.8 & 1.7 & 4.33 & 0.22 & 0.75 & 0.16 & IRAS 10276-5711 & 1 \\
\hline $285.62-0.86$ & 157.67 & -58.91 & $\ldots$ & $\ldots$ & $\ldots$ & $\ldots$ & 2.8 & 1.8 & 1.88 & 0.67 & $\ldots$ & $\ldots$ & PMN J1030-5853 & 0 \\
\hline
\end{tabular}


Table 2

(Continued)

\begin{tabular}{|c|c|c|c|c|c|c|c|c|c|c|c|c|c|c|}
\hline \multirow[t]{2}{*}{ Source } & \multirow[b]{2}{*}{$\begin{array}{l}\text { R.A. } \\
\text { (deg) }\end{array}$} & \multirow[b]{2}{*}{$\begin{array}{l}\text { Decl. } \\
\text { (deg) }\end{array}$} & \multicolumn{4}{|c|}{$100 \mathrm{GHz}$} & \multicolumn{4}{|c|}{$150 \mathrm{GHz}$} & \multirow[t]{2}{*}{$\alpha_{I}$} & \multirow[t]{2}{*}{$\delta \alpha_{I}$} & \multirow[t]{2}{*}{ Alt. Name } & \multirow[t]{2}{*}{ WC } \\
\hline & & & $\begin{array}{c}\sigma_{\text {maj }} \\
(\operatorname{arcmin})\end{array}$ & $\begin{array}{c}\sigma_{\min } \\
(\operatorname{arcmin})\end{array}$ & $\begin{array}{c}I \\
(\mathrm{Jy})\end{array}$ & $\begin{array}{l}\delta I \\
(\mathrm{Jy})\end{array}$ & $\begin{array}{c}\sigma_{\text {maj }} \\
(\operatorname{arcmin})\end{array}$ & $\begin{array}{c}\sigma_{\min } \\
(\operatorname{arcmin})\end{array}$ & $\begin{array}{c}I \\
(\mathrm{Jy})\end{array}$ & $\begin{array}{l}\delta I \\
(\mathrm{Jy})\end{array}$ & & & & \\
\hline $285.28-0.05$ & 157.89 & -58.05 & 3.4 & 2.4 & 17.77 & 0.18 & 2.6 & 1.8 & 19.57 & 0.22 & 0.21 & 0.03 & IRAS 10295-5746 & 1 \\
\hline $286.40-1.38$ & 158.47 & -59.76 & $\ldots$ & $\ldots$ & $\ldots$ & $\ldots$ & 2.9 & 1.8 & 3.30 & 0.69 & $\ldots$ & $\ldots$ & none & 0 \\
\hline $285.45+0.73$ & 158.91 & -57.46 & $\ldots$ & $\ldots$ & $\ldots$ & $\ldots$ & 3.6 & 1.8 & 1.61 & 1.16 & $\ldots$ & $\ldots$ & none & 0 \\
\hline $286.22-0.18$ & 159.33 & -58.63 & 5.6 & 4.8 & 17.48 & 0.38 & 4.6 & 2.9 & 9.30 & 0.50 & -1.37 & 0.13 & none & 0 \\
\hline $286.09+0.16$ & 159.43 & -58.27 & $\ldots$ & $\ldots$ & $\ldots$ & $\ldots$ & 4.3 & 2.4 & 2.93 & 0.72 & $\ldots$ & $\ldots$ & IRAS 10339-5745 & 0 \\
\hline $286.38-0.27$ & 159.51 & -58.79 & $\ldots$ & $\ldots$ & $\ldots$ & $\ldots$ & 4.9 & 2.4 & 6.36 & 0.57 & $\ldots$ & $\ldots$ & none & 0 \\
\hline $286.23+0.16$ & 159.66 & -58.34 & 3.0 & 2.4 & 1.31 & 0.26 & 2.6 & 1.9 & 5.48 & 0.24 & 3.12 & 0.44 & IRAS $10365-5803$ & 1 \\
\hline $286.99-0.75$ & 160.10 & -59.50 & 4.0 & 2.3 & 1.70 & 0.67 & $\ldots$ & $\ldots$ & $\ldots$ & $\ldots$ & $\ldots$ & $\ldots$ & IRAS 10381-5912 & 0 \\
\hline $287.20-0.74$ & 160.47 & -59.60 & $\ldots$ & $\ldots$ & $\ldots$ & $\ldots$ & 8.0 & 2.2 & 7.52 & 1.56 & $\ldots$ & $\ldots$ & IRAS 10382-5904 & 0 \\
\hline $287.39-0.65$ & 160.88 & -59.61 & $\ldots$ & $\ldots$ & $\ldots$ & $\ldots$ & 3.8 & 2.8 & 71.37 & 0.51 & $\ldots$ & $\ldots$ & IRAS $10400-5905$ & 0 \\
\hline $287.65-0.97$ & 161.05 & -60.02 & 5.2 & 4.9 & 11.56 & 1.19 & $\ldots$ & $\ldots$ & $\ldots$ & $\ldots$ & $\ldots$ & $\ldots$ & IRAS 10419-5942 & 1 \\
\hline $287.50-0.65$ & 161.07 & -59.66 & 11.9 & 3.7 & 204.69 & 0.74 & $\ldots$ & $\ldots$ & $\ldots$ & $\ldots$ & $\ldots$ & $\ldots$ & IRAS 10419-5925 & 1 \\
\hline $287.21+0.04$ & 161.21 & -58.91 & 8.7 & 2.6 & 7.71 & 0.43 & 4.2 & 1.4 & 1.63 & 0.48 & -3.38 & 0.65 & IRAS $10431-5839$ & 0 \\
\hline $287.59-0.65$ & 161.24 & -59.70 & $\ldots$ & $\ldots$ & $\ldots$ & $\ldots$ & 3.4 & 2.0 & 67.75 & 0.41 & $\ldots$ & $\ldots$ & none & 0 \\
\hline $287.80-0.84$ & 161.42 & -59.97 & 4.3 & 2.7 & 4.06 & 0.83 & 6.4 & 1.7 & 6.07 & 5.03 & 0.88 & 1.86 & IRAS 10439-5941 & 0 \\
\hline $287.73-0.66$ & 161.47 & -59.77 & 4.4 & 3.2 & 8.22 & 1.68 & $\ldots$ & $\ldots$ & & $\ldots$ & $\ldots$ & $\ldots$ & IRAS $10443-5930$ & 0 \\
\hline $287.92-1.01$ & 161.48 & -60.17 & $\ldots$ & $\ldots$ & $\ldots$ & $\ldots$ & 3.3 & 2.1 & 5.92 & 0.86 & $\ldots$ & $\ldots$ & none & 0 \\
\hline $287.26+0.34$ & 161.57 & -58.67 & 4.4 & 2.6 & 2.06 & 0.67 & $\ldots$ & $\ldots$ & $\ldots$ & $\ldots$ & $\ldots$ & $\ldots$ & IRAS 10441-5823 & 1 \\
\hline $287.53-0.16$ & 161.57 & -59.24 & 5.5 & 2.3 & 2.25 & 0.73 & 4.5 & 1.1 & 1.76 & 0.59 & -0.54 & 1.02 & IRAS $10443-5857$ & 0 \\
\hline $287.44+0.13$ & 161.68 & -58.94 & 18.7 & 3.2 & 9.57 & 2.71 & $\ldots$ & $\ldots$ & $\ldots$ & $\ldots$ & $\ldots$ & $\ldots$ & IRAS 10446-5839 & 0 \\
\hline $287.96-0.86$ & 161.70 & -60.06 & 10.8 & 3.6 & 38.05 & 1.11 & 5.5 & 3.4 & 15.58 & 1.38 & -1.94 & 0.20 & IRAS 10445-5947 & 1 \\
\hline $287.67+0.12$ & 162.07 & -59.05 & $\ldots$ & $\ldots$ & $\ldots$ & $\ldots$ & 3.8 & 3.6 & 3.98 & 3.57 & $\ldots$ & $\ldots$ & IRAS $10444-5829$ & 0 \\
\hline $287.75+0.05$ & 162.15 & -59.16 & 6.5 & 2.0 & 1.75 & 21.10 & $\ldots$ & $\ldots$ & $\ldots$ & $\ldots$ & $\ldots$ & $\ldots$ & none & 0 \\
\hline $287.91+0.01$ & 162.39 & -59.26 & 14.5 & 7.7 & 10.94 & 6.02 & $\ldots$ & $\ldots$ & $\ldots$ & $\ldots$ & $\ldots$ & $\ldots$ & none & 0 \\
\hline $286.88+2.88$ & 163.07 & -56.23 & $\ldots$ & $\ldots$ & $\ldots$ & & 1.8 & 1.2 & 0.98 & 0.34 & $\ldots$ & $\ldots$ & none & 0 \\
\hline $289.09-0.38$ & 164.15 & -60.13 & 4.4 & 3.1 & 8.75 & 0.75 & 3.4 & 2.2 & 8.35 & 0.91 & -0.10 & 0.30 & none & 0 \\
\hline $292.07+1.74$ & 171.21 & -59.29 & 2.8 & 2.8 & 3.67 & 0.30 & 3.6 & 2.5 & 4.53 & 48.53 & 0.46 & 23.34 & IRAS $11225-5858$ & 0 \\
\hline $314.26+0.41$ & 216.27 & -60.39 & 5.5 & 4.1 & 9.04 & 8.85 & $\ldots$ & $\ldots$ & $\ldots$ & $\ldots$ & $\ldots$ & $\ldots$ & IRAS 14212-6009 & 1 \\
\hline $314.22+0.27$ & 216.30 & -60.53 & $\ldots$ & $\ldots$ & $\ldots$ & $\ldots$ & 3.2 & 1.5 & 4.98 & 1.99 & $\ldots$ & $\ldots$ & IRAS $14177-6005$ & 0 \\
\hline $316.80-0.07$ & 221.34 & -59.84 & 3.7 & 2.4 & 32.49 & 0.36 & 3.1 & 1.8 & 35.79 & 0.34 & 0.21 & 0.03 & IRAS 14416-5937 & 1 \\
\hline $316.99+0.27$ & 221.41 & -59.45 & 5.5 & 3.4 & 12.35 & 0.39 & 5.1 & 2.9 & 13.15 & 0.49 & 0.14 & 0.11 & IRAS 14418-5912 & 1 \\
\hline $317.05+0.04$ & 221.71 & -59.63 & $\ldots$ & $\ldots$ & $\ldots$ & $\ldots$ & 7.5 & 2.4 & 4.90 & 2.62 & $\ldots$ & $\ldots$ & none & 0 \\
\hline $317.31+0.22$ & 222.02 & -59.36 & $\ldots$ & $\ldots$ & $\ldots$ & $\ldots$ & 4.5 & 2.6 & 4.94 & 0.61 & $\ldots$ & $\ldots$ & IRAS $14405-5855$ & 0 \\
\hline $317.34+0.16$ & 222.12 & -59.40 & 10.7 & 3.8 & 13.83 & 0.72 & $\ldots$ & $\ldots$ & & $\ldots$ & $\ldots$ & $\ldots$ & none & 0 \\
\hline $317.39+0.09$ & 222.27 & -59.44 & $\ldots$ & $\ldots$ & $\ldots$ & $\ldots$ & 4.0 & 2.8 & 8.21 & 0.45 & $\ldots$ & $\ldots$ & IRAS 14418-5901 & 0 \\
\hline $317.71+0.08$ & 222.84 & -59.31 & 4.5 & 2.1 & 1.08 & 1.02 & 2.9 & 2.2 & 3.26 & 0.35 & 2.40 & 2.06 & IRAS 14473-5904 & 1 \\
\hline $317.77-0.03$ & 223.04 & -59.38 & $\ldots$ & $\ldots$ & $\ldots$ & $\ldots$ & 3.4 & 2.4 & 1.89 & 1.13 & $\ldots$ & $\ldots$ & none & 0 \\
\hline $317.85+0.13$ & 223.05 & -59.20 & 3.9 & 3.1 & 2.35 & 0.44 & 2.8 & 1.7 & 1.90 & 0.30 & -0.47 & 0.53 & IRAS $14482-5857$ & 1 \\
\hline $318.05+0.06$ & 223.46 & -59.17 & $\ldots$ & $\ldots$ & $\ldots$ & $\ldots$ & 2.5 & 1.7 & 2.72 & 0.22 & $\ldots$ & $\ldots$ & IRAS 14464-5846 & 0 \\
\hline $317.90-0.28$ & 223.49 & -59.55 & $\ldots$ & $\ldots$ & $\ldots$ & $\ldots$ & 2.8 & 2.0 & 2.38 & 0.40 & $\ldots$ & & IRAS $14466-5910$ & 0 \\
\hline $318.35+0.08$ & 223.95 & -59.02 & 21.1 & 0.6 & 1.56 & 22.21 & $\ldots$ & $\ldots$ & $\ldots$ & & & & none & 0 \\
\hline $318.00-0.76$ & 224.10 & -59.93 & 2.0 & 1.4 & 0.61 & 0.35 & & $\ldots$ & $\ldots$ & $\ldots$ & $\ldots$ & $\ldots$ & IRAS 14524-5944 & 1 \\
\hline $318.22-0.60$ & 224.35 & -59.68 & 5.3 & 4.8 & 3.99 & 3.53 & 2.8 & 2.0 & 1.50 & 0.54 & -2.13 & 2.08 & IRAS $14533-5927$ & 1 \\
\hline $318.79-0.15$ & 224.92 & -59.01 & 3.4 & 1.8 & 1.13 & 1.56 & 2.5 & 1.5 & 2.22 & 0.21 & 1.47 & 3.01 & IRAS 14557-5849 & 1 \\
\hline $319.35+0.85$ & 224.96 & -57.87 & $\ldots$ & $\ldots$ & $\ldots$ & the & 6.1 & 2.1 & 3.62 & 0.43 & $\ldots$ & $\ldots$ & IRAS $14521-5728$ & 0 \\
\hline $318.93-0.19$ & 225.19 & -58.99 & 3.3 & 2.2 & 3.19 & 0.20 & 3.0 & 1.6 & 4.32 & 0.21 & 0.66 & 0.17 & IRAS $14567-5846$ & 1 \\
\hline $319.23+0.21$ & 225.34 & -58.49 & $\ldots$ & $\ldots$ & $\ldots$ & $\ldots$ & 3.7 & 1.3 & 0.91 & 0.40 & $\ldots$ & $\ldots$ & IRAS 14536-5805 & 0 \\
\hline $319.18-0.39$ & 225.81 & -59.04 & 5.0 & 4.4 & 11.36 & 0.38 & 4.7 & 3.1 & 10.17 & 0.59 & -0.24 & 0.15 & IRAS $14593-5852$ & 1 \\
\hline $319.40-0.03$ & 225.83 & -58.62 & 4.5 & 2.4 & 8.06 & 0.21 & 3.6 & 1.7 & 7.59 & 0.21 & -0.13 & 0.08 & IRAS 14594-5824 & 1 \\
\hline $319.88+0.76$ & 225.91 & -57.69 & 3.4 & 2.0 & 2.41 & 0.16 & 2.8 & 1.8 & 4.60 & 0.18 & 1.41 & 0.17 & IRAS $14597-5728$ & 1 \\
\hline $320.17+0.78$ & 226.36 & -57.54 & 3.2 & 2.5 & 9.85 & 0.16 & 2.6 & 2.2 & 13.36 & 0.19 & 0.66 & 0.05 & IRAS $15015-5720$ & 1 \\
\hline $320.25+0.41$ & 226.84 & -57.82 & 3.4 & 2.5 & 2.98 & 0.18 & 2.7 & 2.0 & 5.12 & 0.19 & 1.18 & 0.15 & IRAS 15033-5736 & 0 \\
\hline $320.40+0.12$ & 227.36 & -57.99 & 4.1 & 2.5 & 3.31 & 0.20 & 4.2 & 2.0 & 6.10 & 0.25 & 1.33 & 0.16 & none & 0 \\
\hline $320.11-0.50$ & 227.47 & -58.68 & 5.7 & 1.9 & 1.26 & 1.11 & $\ldots$ & $\ldots$ & $\ldots$ & $\ldots$ & $\ldots$ & $\ldots$ & IRAS $15061-5828$ & 1 \\
\hline $321.20+1.30$ & 227.53 & -56.58 & $\ldots$ & $\ldots$ & $\ldots$ & $\ldots$ & 2.8 & 1.7 & 2.51 & 0.21 & $\ldots$ & $\ldots$ & none & 0 \\
\hline $320.33-0.21$ & 227.56 & -58.32 & 3.6 & 2.3 & 6.57 & 0.19 & 2.7 & 1.6 & 7.63 & 0.18 & 0.33 & 0.08 & IRAS 15061-5806 & 1 \\
\hline $320.27-0.34$ & 227.58 & -58.45 & 3.6 & 2.6 & 7.42 & 0.21 & 3.3 & 1.7 & 7.87 & 0.21 & 0.13 & 0.08 & IRAS 15061-5814 & 1 \\
\hline $320.75+0.19$ & 227.85 & -57.75 & 5.9 & 4.0 & 11.58 & 0.31 & 5.5 & 3.1 & 13.59 & 0.37 & 0.35 & 0.08 & IRAS $15076-5730$ & 1 \\
\hline $320.36-1.08$ & 228.46 & -59.04 & 3.3 & 2.6 & 1.59 & 0.49 & $\ldots$ & $\ldots$ & $\ldots$ & $\ldots$ & $\ldots$ & $\ldots$ & IRAS 15098-5849 & 0 \\
\hline $321.72+1.16$ & 228.47 & -56.43 & 3.3 & 2.4 & 3.80 & 0.18 & 2.6 & 1.7 & 6.60 & 0.18 & 1.20 & 0.12 & IRAS $15100-5613$ & 1 \\
\hline $320.89-0.40$ & 228.66 & -58.19 & 4.2 & 3.0 & 1.43 & 0.64 & 3.2 & 1.8 & 3.13 & 0.23 & 1.70 & 0.98 & IRAS $15107-5800$ & 1 \\
\hline $322.09+1.41$ & 228.81 & -56.02 & 2.5 & 2.3 & 0.89 & 0.30 & $\ldots$ & $\ldots$ & $\ldots$ & $\ldots$ & $\ldots$ & $\ldots$ & IRAS $15112-5550$ & 0 \\
\hline $321.09-0.54$ & 229.12 & -58.21 & 5.7 & 2.5 & 14.61 & 0.26 & 5.8 & 2.1 & 16.01 & 0.32 & 0.20 & 0.06 & IRAS $15122-5801$ & 1 \\
\hline $321.39-0.34$ & 229.40 & -57.87 & $\ldots$ & $\ldots$ & $\ldots$ & $\ldots$ & 3.2 & 1.4 & 1.23 & 0.46 & $\ldots$ & $\ldots$ & none & 0 \\
\hline
\end{tabular}


Table 2

(Continued)

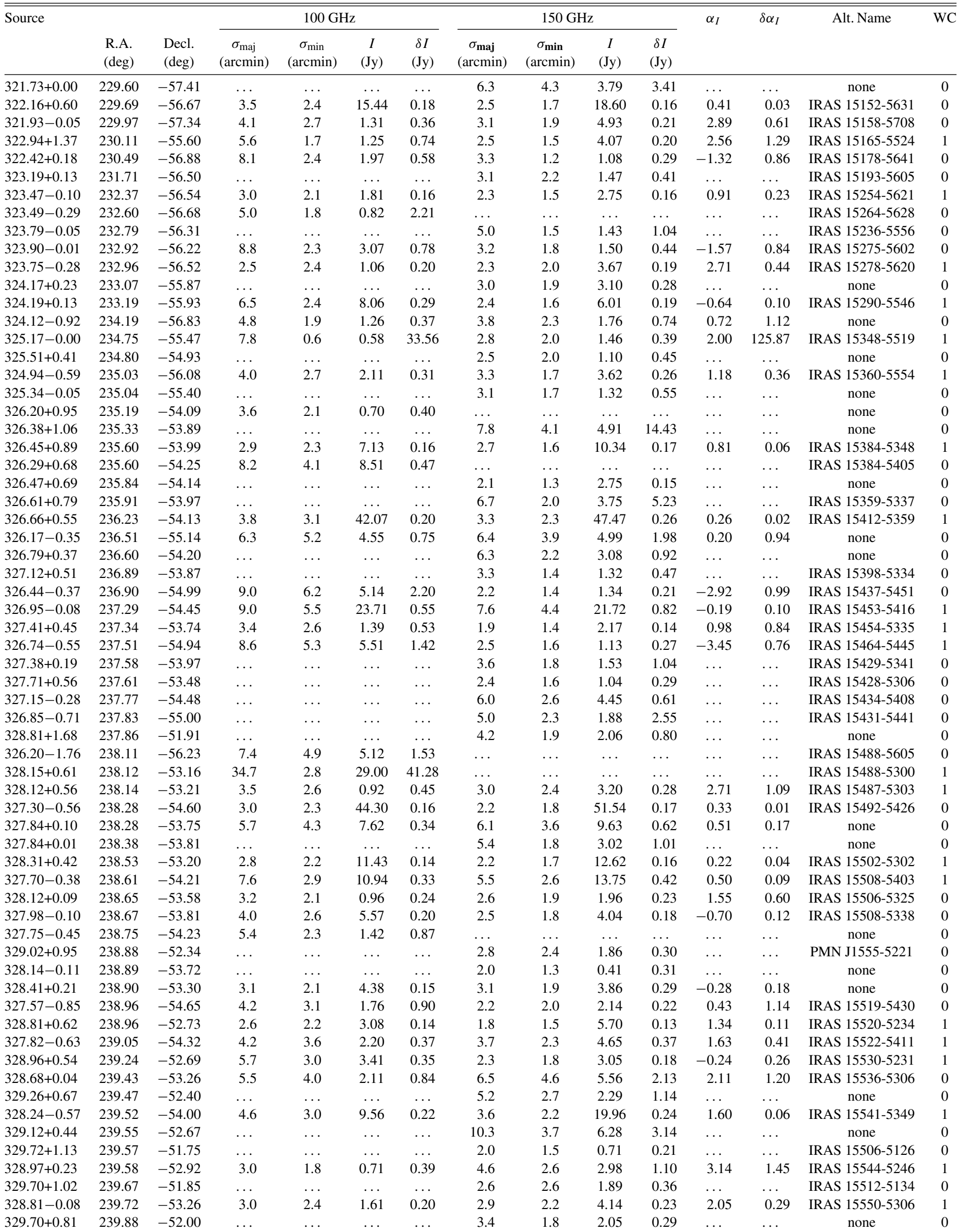


Table 2

(Continued)

\begin{tabular}{|c|c|c|c|c|c|c|c|c|c|c|c|c|c|c|}
\hline \multirow[t]{2}{*}{ Source } & \multirow[b]{2}{*}{$\begin{array}{l}\text { R.A. } \\
\text { (deg) }\end{array}$} & \multirow[b]{2}{*}{$\begin{array}{l}\text { Decl. } \\
\text { (deg) }\end{array}$} & \multicolumn{4}{|c|}{$100 \mathrm{GHz}$} & \multicolumn{4}{|c|}{$150 \mathrm{GHz}$} & \multirow[t]{2}{*}{$\alpha_{I}$} & \multirow[t]{2}{*}{$\delta \alpha_{I}$} & \multirow[t]{2}{*}{ Alt. Name } & \multirow[t]{2}{*}{ WC } \\
\hline & & & $\begin{array}{c}\sigma_{\text {maj }} \\
(\operatorname{arcmin})\end{array}$ & $\begin{array}{c}\sigma_{\min } \\
(\operatorname{arcmin})\end{array}$ & $\begin{array}{c}I \\
(\mathrm{Jy})\end{array}$ & $\begin{array}{l}\delta I \\
(\mathrm{Jy})\end{array}$ & $\begin{array}{c}\sigma_{\text {maj }} \\
(\operatorname{arcmin})\end{array}$ & $\begin{array}{c}\sigma_{\min } \\
(\operatorname{arcmin})\end{array}$ & $\begin{array}{c}I \\
(\mathrm{Jy})\end{array}$ & $\begin{array}{c}\delta I \\
(\mathrm{Jy})\end{array}$ & & & & \\
\hline $329.46+0.50$ & 239.91 & -52.40 & $\ldots$ & $\ldots$ & & & 2.3 & 1.6 & 1.93 & 0.17 & & & none & 0 \\
\hline $328.57-0.54$ & 239.91 & -53.76 & 2.7 & 2.2 & 9.34 & 0.14 & 2.0 & 1.6 & 11.00 & 0.15 & 0.36 & 0.04 & IRAS $15557-5337$ & 0 \\
\hline $330.01+1.03$ & 240.02 & -51.64 & $\ldots$ & $\ldots$ & $\ldots$ & $\ldots$ & 3.0 & 2.1 & 1.92 & 0.36 & $\ldots$ & $\ldots$ & none & 0 \\
\hline $329.03-0.21$ & 240.15 & -53.22 & $\ldots$ & $\ldots$ & $\ldots$ & $\ldots$ & 2.7 & 1.5 & 4.06 & 0.23 & $\ldots$ & $\ldots$ & IRAS $15527-5258$ & 0 \\
\hline $329.34+0.13$ & 240.15 & -52.76 & $\ldots$ & $\ldots$ & $\ldots$ & $\ldots$ & 1.8 & 1.5 & 4.43 & 0.13 & $\ldots$ & $\ldots$ & IRAS $15530-5228$ & 0 \\
\hline $330.01+0.88$ & 240.18 & -51.75 & $\ldots$ & $\ldots$ & $\ldots$ & $\ldots$ & 4.1 & 2.8 & 2.12 & 1.08 & $\ldots$ & $\ldots$ & none & 0 \\
\hline $329.40+0.15$ & 240.21 & -52.71 & 8.1 & 2.9 & 9.80 & 0.33 & $\ldots$ & $\ldots$ & $\ldots$ & $\ldots$ & $\ldots$ & $\ldots$ & IRAS $15570-5234$ & 0 \\
\hline $329.48+0.18$ & 240.28 & -52.63 & $\ldots$ & $\ldots$ & $\ldots$ & $\ldots$ & 6.6 & 3.8 & 9.91 & 0.68 & $\ldots$ & $\ldots$ & none & 0 \\
\hline $329.78+0.39$ & 240.43 & -52.27 & $\ldots$ & $\ldots$ & $\ldots$ & $\ldots$ & 15.1 & 5.7 & 12.15 & 12.56 & $\ldots$ & $\ldots$ & IRAS $15543-5200$ & 0 \\
\hline $329.18-0.33$ & 240.46 & -53.21 & $\ldots$ & $\ldots$ & $\ldots$ & $\ldots$ & 8.5 & 2.0 & 4.19 & 0.82 & $\ldots$ & $\ldots$ & IRAS $15543-5258$ & 0 \\
\hline $329.42-0.18$ & 240.60 & -52.94 & $\ldots$ & $\ldots$ & $\ldots$ & $\ldots$ & 2.1 & 1.7 & 1.33 & 0.17 & $\ldots$ & $\ldots$ & IRAS 15548-5239 & 0 \\
\hline $330.29+0.62$ & 240.80 & -51.76 & 5.0 & 2.8 & 1.57 & 0.43 & 7.2 & 2.7 & 5.68 & 0.62 & 2.80 & 0.64 & IRAS 15592-5139 & 0 \\
\hline $329.41-0.48$ & 240.91 & -53.17 & $\ldots$ & $\ldots$ & $\ldots$ & $\ldots$ & 1.6 & 1.3 & 0.83 & 0.14 & $\ldots$ & $\ldots$ & IRAS $15559-5251$ & 0 \\
\hline $330.03-0.06$ & 241.23 & -52.44 & 2.6 & 2.3 & 0.85 & 0.27 & 3.1 & 1.7 & 1.32 & 0.43 & 0.94 & 0.98 & IRAS $16010-5218$ & 1 \\
\hline $332.40+2.46$ & 241.35 & -48.99 & $\ldots$ & $\ldots$ & $\ldots$ & $\ldots$ & 3.2 & 1.4 & 1.73 & 0.21 & $\ldots$ & $\ldots$ & none & 0 \\
\hline $332.30+2.27$ & 241.43 & -49.19 & $\ldots$ & $\ldots$ & $\ldots$ & $\ldots$ & 2.1 & 1.5 & 1.00 & 0.19 & $\ldots$ & $\ldots$ & none & 0 \\
\hline $331.32+1.05$ & 241.57 & -50.76 & 5.3 & 2.8 & 5.64 & 0.24 & 5.3 & 2.0 & 7.35 & 0.31 & 0.57 & 0.13 & IRAS $16026-5035$ & 1 \\
\hline $330.61+0.14$ & 241.70 & -51.91 & 9.8 & 3.3 & 2.58 & 1.42 & $\ldots$ & $\ldots$ & $\ldots$ & $\ldots$ & $\ldots$ & $\ldots$ & none & 0 \\
\hline $330.37-0.21$ & 241.79 & -52.33 & $\ldots$ & $\ldots$ & $\ldots$ & $\ldots$ & 2.9 & 1.5 & 1.18 & 7.05 & $\ldots$ & $\ldots$ & IRAS $15596-5203$ & 0 \\
\hline $330.30-0.41$ & 241.93 & -52.53 & 2.7 & 1.9 & 1.36 & 0.19 & 1.6 & 1.4 & 1.42 & 0.13 & 0.11 & 0.36 & IRAS 16037-5223 & 1 \\
\hline $331.35+0.51$ & 242.18 & -51.13 & 3.4 & 3.1 & 3.02 & 0.21 & 4.6 & 3.0 & 4.54 & 0.48 & 0.89 & 0.27 & PMN J1608-5107 & 0 \\
\hline $331.13+0.11$ & 242.37 & -51.58 & $\ldots$ & $\ldots$ & $\ldots$ & $\ldots$ & 4.5 & 2.0 & 2.82 & 0.36 & $\ldots$ & $\ldots$ & none & 0 \\
\hline $330.69-0.40$ & 242.39 & -52.25 & 3.2 & 2.5 & 3.01 & 0.18 & 2.6 & 1.6 & 2.85 & 0.18 & -0.12 & 0.19 & IRAS 16056-5207 & 1 \\
\hline $333.04+2.06$ & 242.49 & -48.85 & 14.5 & 3.9 & 4.23 & 8.09 & $\ldots$ & $\ldots$ & $\ldots$ & $\ldots$ & $\ldots$ & $\ldots$ & none & 0 \\
\hline 330.98-0.19 & 242.51 & -51.91 & 3.9 & 2.7 & 14.47 & 0.20 & 2.1 & 1.7 & 14.64 & 0.17 & 0.03 & 0.04 & IRAS $16060-5146$ & 1 \\
\hline $331.63+0.51$ & 242.51 & -50.95 & $\ldots$ & $\ldots$ & $\ldots$ & $\ldots$ & 2.5 & 1.5 & 1.31 & 0.21 & $\ldots$ & $\ldots$ & IRAS $16027-5044$ & 0 \\
\hline $331.71+0.58$ & 242.52 & -50.84 & $\ldots$ & $\ldots$ & $\ldots$ & $\ldots$ & 2.3 & 1.6 & 1.64 & 0.18 & $\ldots$ & $\ldots$ & IRAS 16026-5035 & 1 \\
\hline $330.87-0.38$ & 242.59 & -52.12 & 3.1 & 2.6 & 10.36 & 0.17 & 2.3 & 1.7 & 12.38 & 0.16 & 0.39 & 0.04 & IRAS $16065-5158$ & 1 \\
\hline $331.38+0.13$ & 242.63 & -51.39 & $\ldots$ & $\ldots$ & $\ldots$ & $\ldots$ & 2.2 & 1.5 & 0.91 & 0.26 & $\ldots$ & $\ldots$ & IRAS 16032-5107 & 0 \\
\hline $332.94+1.76$ & 242.69 & -49.14 & 4.0 & 2.7 & 1.24 & 0.30 & 2.9 & 2.1 & 1.71 & 0.48 & 0.70 & 0.81 & IRAS $16069-4858$ & 1 \\
\hline $331.12-0.25$ & 242.74 & -51.85 & $\ldots$ & $\ldots$ & $\ldots$ & $\ldots$ & 2.4 & 1.7 & 3.95 & 0.25 & $\ldots$ & $\ldots$ & IRAS 16034-5132 & 0 \\
\hline $331.37-0.02$ & 242.79 & -51.52 & $\ldots$ & $\ldots$ & $\ldots$ & $\ldots$ & 2.2 & 2.0 & 4.42 & 0.18 & $\ldots$ & $\ldots$ & none & 0 \\
\hline $333.41+2.11$ & 242.86 & -48.57 & $\ldots$ & $\ldots$ & $\ldots$ & $\ldots$ & 2.2 & 1.9 & 1.32 & 0.24 & $\ldots$ & $\ldots$ & PMN J1611-4830 & 0 \\
\hline $331.27-0.20$ & 242.87 & -51.72 & 2.1 & 1.9 & 2.09 & 0.12 & 1.7 & 1.4 & 3.10 & 0.12 & 0.86 & 0.15 & IRAS $16076-5134$ & 1 \\
\hline $333.36+2.03$ & 242.88 & -48.66 & $\ldots$ & $\ldots$ & $\ldots$ & $\ldots$ & 5.1 & 2.6 & 3.07 & 0.67 & $\ldots$ & $\ldots$ & IRAS $16040-4827$ & 0 \\
\hline $332.06+0.48$ & 243.04 & -50.68 & $\ldots$ & $\ldots$ & $\ldots$ & $\ldots$ & 1.9 & 1.6 & 0.86 & 0.23 & $\ldots$ & $\ldots$ & PMN J1612-5039 & 0 \\
\hline $331.52-0.10$ & 243.05 & -51.46 & 3.1 & 2.6 & 31.25 & 0.17 & 2.4 & 2.1 & 36.19 & 0.18 & 0.32 & 0.02 & IRAS 16086-5119 & 1 \\
\hline $331.13-0.52$ & 243.05 & -52.04 & 3.6 & 2.7 & 6.15 & 0.18 & 2.8 & 2.1 & 9.19 & 0.19 & 0.87 & 0.08 & IRAS 16083-5154 & 1 \\
\hline $331.34-0.36$ & 243.12 & -51.78 & 3.4 & 2.5 & 7.05 & 0.18 & 3.8 & 2.0 & 9.29 & 0.27 & 0.60 & 0.08 & IRAS $16085-5138$ & 1 \\
\hline $331.86+0.18$ & 243.15 & -51.03 & $\ldots$ & $\ldots$ & $\ldots$ & $\ldots$ & 4.9 & 1.7 & 1.61 & 27.99 & $\ldots$ & $\ldots$ & IRAS 16048-5049 & 0 \\
\hline $331.74+0.02$ & 243.17 & -51.23 & $\ldots$ & $\ldots$ & & $\ldots$ & 4.7 & 2.9 & 3.00 & 1.53 & & $\ldots$ & none & 0 \\
\hline $331.90-0.12$ & 243.51 & -51.23 & $\ldots$ & $\ldots$ & $\cdots$ & $\ldots$ & 10.1 & 4.5 & 12.78 & 1.81 & & $\ldots$ & IRAS $16065-5056$ & 0 \\
\hline $332.12+0.04$ & 243.59 & -50.95 & $\ldots$ & $\ldots$ & $\ldots$ & $\ldots$ & 11.2 & 2.0 & 4.42 & 17.06 & $\ldots$ & $\ldots$ & IRAS $16069-5042$ & 0 \\
\hline $332.98+0.76$ & 243.79 & -49.84 & 3.0 & 2.5 & 8.15 & 0.16 & 2.7 & 2.0 & 12.32 & 0.18 & 0.90 & 0.05 & IRAS 16112-4943 & 1 \\
\hline $332.27-0.08$ & 243.90 & -50.94 & 5.3 & 2.9 & 1.82 & 0.47 & 5.0 & 1.7 & 4.57 & 0.30 & 2.00 & 0.58 & IRAS $16119-5048$ & 1 \\
\hline $332.43+0.05$ & 243.94 & -50.74 & 4.7 & 2.9 & 1.97 & 0.31 & 4.3 & 2.4 & 3.18 & 0.44 & 1.05 & 0.46 & IRAS 16119-5034 & 0 \\
\hline $332.65+0.21$ & 244.02 & -50.47 & $\ldots$ & $\ldots$ & $\ldots$ & $\ldots$ & 4.0 & 1.8 & 1.64 & 0.63 & $\ldots$ & $\ldots$ & IRAS $16088-5012$ & 0 \\
\hline $332.59+0.04$ & 244.13 & -50.63 & $\ldots$ & $\ldots$ & $\ldots$ & $\ldots$ & 2.3 & 1.2 & 0.62 & 0.31 & $\ldots$ & $\ldots$ & IRAS 16092-5021 & 0 \\
\hline $332.15-0.46$ & 244.17 & -51.30 & 2.8 & 2.3 & 12.02 & 0.14 & 2.3 & 1.7 & 12.56 & 0.16 & 0.09 & 0.04 & IRAS 16128-5109 & 1 \\
\hline $332.88+0.26$ & 244.22 & -50.27 & $\ldots$ & $\ldots$ & $\ldots$ & $\ldots$ & 3.7 & 2.3 & 2.36 & 21.62 & $\ldots$ & $\ldots$ & IRAS 16096-4959 & 0 \\
\hline $332.54-0.15$ & 244.27 & -50.80 & 3.5 & 2.2 & 3.41 & 0.16 & 3.3 & 1.7 & 5.61 & 0.21 & 1.08 & 0.13 & IRAS 16132-5039 & 1 \\
\hline $332.76-0.02$ & 244.38 & -50.55 & $\ldots$ & $\ldots$ & $\ldots$ & $\ldots$ & 1.8 & 1.6 & 1.42 & 0.15 & $\ldots$ & $\ldots$ & IRAS 16099-5018 & 0 \\
\hline $332.40-0.40$ & 244.40 & -51.07 & 3.2 & 2.5 & 1.52 & 0.22 & $\ldots$ & $\ldots$ & $\ldots$ & $\ldots$ & $\cdots$ & $\ldots$ & IRAS $16135-5058$ & 0 \\
\hline $332.29-0.57$ & 244.46 & -51.28 & $\ldots$ & $\ldots$ & $\ldots$ & $\ldots$ & 3.9 & 1.8 & 2.55 & 0.29 & $\cdots$ & $\ldots$ & IRAS 16103-5104 & 0 \\
\hline $333.06-0.00$ & 244.71 & -50.33 & $\ldots$ & $\ldots$ & $\ldots$ & $\ldots$ & 7.1 & 5.1 & 11.45 & 1.80 & $\ldots$ & $\ldots$ & none & 0 \\
\hline $331.92-1.17$ & 244.71 & -51.97 & 6.3 & 3.8 & 2.16 & 2.32 & 3.2 & 2.7 & 1.65 & 0.82 & -0.59 & 2.58 & IRAS $16148-5150$ & 0 \\
\hline $333.25+0.04$ & 244.87 & -50.17 & $\ldots$ & $\ldots$ & $\ldots$ & $\ldots$ & 2.4 & 1.7 & 3.52 & 0.19 & $\ldots$ & $\ldots$ & none & 0 \\
\hline $333.16-0.08$ & 244.90 & -50.32 & 8.2 & 4.1 & 17.21 & 0.48 & 3.6 & 2.9 & 16.48 & 0.28 & -0.09 & 0.07 & IRAS 16159-5012 & 1 \\
\hline $332.67-0.64$ & 244.97 & -51.06 & $\ldots$ & $\ldots$ & $\ldots$ & $\ldots$ & 3.3 & 2.1 & 19.91 & 0.21 & $\ldots$ & $\ldots$ & IRAS $16122-5047$ & 0 \\
\hline $332.74-0.61$ & 245.02 & -50.99 & 6.8 & 3.0 & 32.86 & 0.27 & $\ldots$ & $\ldots$ & $\ldots$ & $\ldots$ & $\ldots$ & $\ldots$ & none & 0 \\
\hline $333.73+0.35$ & 245.06 & -49.62 & $\ldots$ & $\ldots$ & $\ldots$ & $\ldots$ & 2.7 & 1.4 & 2.42 & 0.17 & $\ldots$ & $\ldots$ & IRAS $16129-4922$ & 0 \\
\hline $332.82-0.57$ & 245.06 & -50.91 & $\ldots$ & $\ldots$ & $\ldots$ & $\ldots$ & 2.0 & 1.8 & 14.03 & 0.16 & $\ldots$ & $\ldots$ & IRAS 16126-5041 & 1 \\
\hline $333.08-0.47$ & 245.24 & -50.65 & 5.3 & 2.9 & 63.44 & 0.24 & 4.4 & 2.4 & 66.40 & 0.26 & 0.10 & 0.01 & IRAS $16172-5032$ & 1 \\
\hline $333.60+0.04$ & 245.27 & -49.92 & $\ldots$ & $\ldots$ & $\ldots$ & $\ldots$ & 6.4 & 3.1 & 8.17 & 1.22 & $\ldots$ & $\ldots$ & none & 0 \\
\hline
\end{tabular}


Table 2

(Continued)

\begin{tabular}{|c|c|c|c|c|c|c|c|c|c|c|c|c|c|c|}
\hline \multirow[t]{2}{*}{ Source } & \multirow[b]{2}{*}{$\begin{array}{l}\text { R.A. } \\
\text { (deg) }\end{array}$} & \multirow[b]{2}{*}{$\begin{array}{l}\text { Decl. } \\
\text { (deg) }\end{array}$} & \multicolumn{4}{|c|}{$100 \mathrm{GHz}$} & \multicolumn{4}{|c|}{$150 \mathrm{GHz}$} & \multirow[t]{2}{*}{$\alpha_{I}$} & \multirow[t]{2}{*}{$\delta \alpha_{I}$} & \multirow[t]{2}{*}{ Alt. Name } & \multirow[t]{2}{*}{ WC } \\
\hline & & & $\begin{array}{c}\sigma_{\text {maj }} \\
(\operatorname{arcmin})\end{array}$ & $\begin{array}{c}\sigma_{\min } \\
(\operatorname{arcmin})\end{array}$ & $\begin{array}{c}I \\
(\mathrm{Jy})\end{array}$ & $\begin{array}{l}\delta I \\
(\mathrm{Jy})\end{array}$ & $\begin{array}{c}\sigma_{\text {maj }} \\
(\operatorname{arcmin})\end{array}$ & $\begin{array}{c}\sigma_{\min } \\
(\operatorname{arcmin})\end{array}$ & $\begin{array}{c}I \\
(\mathrm{Jy})\end{array}$ & $\begin{array}{l}\delta I \\
(\mathrm{Jy})\end{array}$ & & & & \\
\hline $333.47-0.17$ & 245.35 & -50.17 & $\ldots$ & $\ldots$ & $\ldots$ & $\ldots$ & 1.7 & 1.3 & 1.67 & 0.16 & . & 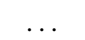 & IRAS 16139-4954 & 0 \\
\hline $332.96-0.68$ & 245.35 & -50.89 & $\ldots$ & $\ldots$ & $\ldots$ & $\ldots$ & 2.0 & 1.6 & 1.90 & 0.23 & $\ldots$ & $\ldots$ & IRAS 16136-5038 & 0 \\
\hline $333.29-0.40$ & 245.40 & -50.46 & 2.8 & 2.7 & 33.29 & 0.16 & 2.1 & 2.0 & 34.65 & 0.16 & 0.09 & 0.01 & IRAS 16177-5018 & 1 \\
\hline $334.52+0.80$ & 245.43 & -48.74 & 3.0 & 2.5 & 1.33 & 0.21 & 3.7 & 2.0 & 1.65 & 0.71 & 0.47 & 0.99 & none & 0 \\
\hline $333.60-0.23$ & 245.56 & -50.11 & 2.7 & 2.4 & 83.41 & 0.15 & 2.0 & 1.6 & 74.39 & 0.14 & -0.25 & 0.01 & IRAS 16183-4958 & 0 \\
\hline $334.19+0.17$ & 245.76 & -49.41 & $\ldots$ & $\ldots$ & $\ldots$ & $\ldots$ & 2.3 & 2.0 & 1.44 & 0.34 & $\ldots$ & $\ldots$ & IRAS 16153-4912 & 0 \\
\hline $334.17+0.02$ & 245.90 & -49.53 & $\ldots$ & $\ldots$ & $\ldots$ & $\ldots$ & 8.6 & 3.0 & 6.42 & 2.00 & $\ldots$ & $\ldots$ & none & 0 \\
\hline $334.21+0.07$ & 245.90 & -49.47 & 8.9 & 5.1 & 5.99 & 0.80 & $\ldots$ & $\ldots$ & & & $\ldots$ & $\ldots$ & IRAS $16197-4920$ & 0 \\
\hline $333.70-0.50$ & 245.96 & -50.24 & 2.8 & 2.3 & 1.62 & 0.18 & 2.4 & 1.8 & 2.16 & 0.20 & 0.62 & 0.31 & IRAS 16200-5007 & 1 \\
\hline $334.65+0.44$ & 245.96 & -48.90 & $\ldots$ & $\ldots$ & $\ldots$ & $\ldots$ & 2.5 & 1.7 & 1.59 & 0.22 & $\ldots$ & $\ldots$ & IRAS 16164-4837 & 1 \\
\hline $334.33+0.06$ & 246.03 & -49.39 & $\ldots$ & $\ldots$ & $\ldots$ & $\ldots$ & 4.4 & 2.5 & 2.45 & 0.60 & $\ldots$ & $\ldots$ & IRAS 16165-4912 & 0 \\
\hline $334.04-0.28$ & 246.09 & -49.84 & $\ldots$ & $\ldots$ & $\ldots$ & $\ldots$ & 3.4 & 1.7 & 1.20 & 0.41 & $\ldots$ & $\ldots$ & PMN J1624-4950 & 0 \\
\hline $334.28-0.12$ & 246.17 & -49.55 & $\ldots$ & $\ldots$ & $\ldots$ & $\ldots$ & 4.5 & 1.1 & 1.25 & 0.36 & $\ldots$ & $\ldots$ & IRAS 16172-4919 & 0 \\
\hline $334.47+0.05$ & 246.19 & -49.30 & $\ldots$ & $\ldots$ & $\ldots$ & $\ldots$ & 6.3 & 2.1 & 2.33 & 1.21 & $\ldots$ & $\ldots$ & none & 0 \\
\hline $334.34-0.26$ & 246.39 & -49.62 & $\ldots$ & $\ldots$ & $\ldots$ & $\ldots$ & 6.2 & 4.1 & 6.30 & 0.98 & $\ldots$ & $\ldots$ & IRAS 16182-4924 & 0 \\
\hline $334.42-0.23$ & 246.45 & -49.53 & 5.2 & 3.9 & 2.37 & 0.56 & 2.6 & 2.2 & 2.45 & 0.27 & 0.07 & 0.56 & IRAS 16220-4925 & 0 \\
\hline $334.70-0.12$ & 246.62 & -49.25 & 4.0 & 2.7 & 2.57 & 0.22 & 2.6 & 1.7 & 2.92 & 0.19 & 0.27 & 0.23 & none & 0 \\
\hline $334.98-0.24$ & 247.06 & -49.14 & 11.4 & 5.5 & 6.87 & 1.49 & 19.0 & 4.9 & 20.83 & 4.84 & 2.42 & 0.69 & IRAS 16244-4901 & 0 \\
\hline $335.28-0.13$ & 247.25 & -48.84 & $\ldots$ & $\ldots$ & $\ldots$ & $\ldots$ & 2.1 & 1.0 & 0.69 & 0.17 & $\ldots$ & $\ldots$ & IRAS 16212-4836 & 0 \\
\hline $334.72-0.67$ & 247.26 & -49.62 & $\ldots$ & $\ldots$ & $\ldots$ & $\ldots$ & 2.0 & 1.2 & 1.00 & 0.22 & $\ldots$ & $\ldots$ & none & 0 \\
\hline $335.17-0.27$ & 247.29 & -49.02 & $\ldots$ & $\ldots$ & $\ldots$ & $\ldots$ & 22.7 & 3.0 & 13.01 & 40.89 & $\ldots$ & $\ldots$ & IRAS 16219-4848 & 0 \\
\hline $335.06-0.43$ & 247.35 & -49.21 & 13.8 & 3.4 & 4.38 & 50.96 & 2.6 & 1.7 & 1.57 & 0.21 & -2.23 & 25.31 & IRAS 16256-4905 & 1 \\
\hline $335.75+0.17$ & 247.42 & -48.29 & 6.4 & 3.5 & 2.90 & 0.59 & 4.9 & 1.6 & 3.60 & 0.39 & 0.47 & 0.50 & none & 0 \\
\hline $335.44-0.22$ & 247.52 & -48.79 & 14.9 & 3.4 & 3.70 & 4.64 & 2.9 & 2.1 & 3.05 & 0.24 & -0.42 & 2.73 & IRAS 16264-4841 & 0 \\
\hline $335.96+0.16$ & 247.64 & -48.15 & 5.6 & 2.3 & 3.29 & 0.28 & 29.2 & 3.2 & 23.18 & 4.95 & 4.25 & 0.50 & IRAS $16267-4800$ & 1 \\
\hline $335.58-0.30$ & 247.75 & -48.74 & $\ldots$ & $\ldots$ & $\ldots$ & $\ldots$ & 1.7 & 1.4 & 1.49 & 0.14 & $\ldots$ & $\ldots$ & IRAS $16235-4832$ & 0 \\
\hline $335.75-0.15$ & 247.76 & -48.52 & 4.1 & 3.1 & 7.88 & 0.22 & 4.0 & 3.1 & 9.95 & 0.34 & 0.51 & 0.10 & IRAS 16274-4823 & 0 \\
\hline $336.12-0.04$ & 248.02 & -48.17 & 7.1 & 3.8 & 3.12 & 1.30 & $\ldots$ & $\ldots$ & $\ldots$ & $\ldots$ & $\ldots$ & $\ldots$ & IRAS $16284-4802$ & 0 \\
\hline $336.37-0.00$ & 248.24 & -47.96 & $\ldots$ & $\ldots$ & $\ldots$ & $\ldots$ & 6.2 & 2.0 & 4.71 & 0.72 & & & IRAS 16257-4744 & 0 \\
\hline $336.37-0.15$ & 248.39 & -48.06 & $\ldots$ & $\ldots$ & $\ldots$ & & 1.8 & 1.7 & 2.18 & 0.17 & & & IRAS 16261-4751 & 0 \\
\hline $335.69-0.82$ & 248.44 & -49.02 & $\ldots$ & $\ldots$ & $\ldots$ & $\ldots$ & 3.4 & 1.9 & 1.66 & 0.40 & $\ldots$ & $\ldots$ & IRAS $16264-4845$ & 1 \\
\hline $336.45-0.21$ & 248.55 & -48.04 & 6.0 & 5.3 & 21.81 & 0.39 & 6.4 & 3.3 & 19.36 & 0.51 & -0.26 & 0.07 & IRAS 16306-4758 & 1 \\
\hline $336.82+0.04$ & 248.64 & -47.61 & 5.1 & 3.4 & 37.37 & 0.24 & 4.3 & 2.7 & 34.75 & 0.29 & -0.16 & 0.02 & none & 0 \\
\hline $336.02-0.84$ & 248.80 & -48.79 & 2.8 & 1.9 & 1.00 & 0.23 & 1.8 & 1.6 & 2.54 & 0.14 & 2.02 & 0.52 & IRAS 16313-4840 & 1 \\
\hline $336.98-0.03$ & 248.89 & -47.53 & $\ldots$ & $\ldots$ & $\ldots$ & $\ldots$ & 2.3 & 1.6 & 4.34 & 0.19 & $\ldots$ & $\ldots$ & none & 0 \\
\hline $336.92-0.17$ & 248.97 & -47.67 & 2.9 & 2.6 & 4.97 & 0.19 & 2.4 & 2.0 & 5.77 & 0.19 & 0.33 & 0.11 & IRAS 16320-4734 & 1 \\
\hline $337.17-0.07$ & 249.12 & -47.41 & $\ldots$ & $\ldots$ & $\ldots$ & $\ldots$ & 4.6 & 2.8 & 18.75 & 0.44 & $\ldots$ & $\ldots$ & IRAS 16294-4712 & 0 \\
\hline $337.37+0.06$ & 249.18 & -47.19 & 3.4 & 2.1 & 1.01 & 0.28 & 5.5 & 3.0 & 6.26 & 0.67 & 3.97 & 0.64 & IRAS 16329-4701 & 0 \\
\hline $337.15-0.16$ & 249.19 & -47.50 & 4.9 & 3.6 & 23.24 & 0.32 & 2.1 & 2.0 & 14.32 & 0.18 & -1.06 & 0.04 & IRAS 16330-4725 & 1 \\
\hline $337.27-0.14$ & 249.28 & -47.39 & $\ldots$ & $\ldots$ & $\ldots$ & $\ldots$ & 3.6 & 3.0 & 14.04 & 0.38 & $\ldots$ & $\ldots$ & none & 0 \\
\hline $337.15-0.40$ & 249.46 & -47.65 & $\ldots$ & $\ldots$ & $\ldots$ & $\ldots$ & 2.9 & 1.0 & 1.15 & 0.28 & $\ldots$ & $\ldots$ & none & 0 \\
\hline $336.30-1.25$ & 249.56 & -48.86 & 3.1 & 3.1 & 3.05 & 0.19 & 2.4 & 2.0 & 7.69 & 0.19 & 2.01 & 0.15 & none & 0 \\
\hline $337.68-0.06$ & 249.61 & -47.03 & 5.2 & 3.9 & 12.84 & 0.28 & 5.7 & 3.5 & 20.99 & 0.49 & 1.07 & 0.07 & IRAS $16348-4654$ & 1 \\
\hline $337.48-0.34$ & 249.71 & -47.37 & 10.4 & 2.8 & 3.93 & 0.92 & $\ldots$ & $\ldots$ & $\cdots$ & $\ldots$ & $\ldots$ & $\ldots$ & IRAS 16351-4718 & 0 \\
\hline $337.41-0.41$ & 249.72 & -47.47 & $\ldots$ & $\ldots$ & $\ldots$ & $\ldots$ & 1.9 & 1.4 & 3.41 & 0.13 & $\ldots$ & $\ldots$ & none & 0 \\
\hline $336.93-0.99$ & 249.89 & -48.21 & $\ldots$ & $\ldots$ & $\ldots$ & $\ldots$ & 3.9 & 1.6 & 1.32 & 0.84 & $\ldots$ & $\ldots$ & IRAS 16323-4801 & 0 \\
\hline $338.02-0.07$ & 249.94 & -46.79 & 6.9 & 6.0 & 32.22 & 0.41 & 6.2 & 5.1 & 50.69 & 0.54 & 0.99 & 0.04 & IRAS 16362-4639 & 1 \\
\hline $336.49-1.48$ & 250.01 & -48.87 & 2.8 & 2.4 & 10.94 & 0.15 & 2.0 & 1.8 & 18.14 & 0.15 & 1.10 & 0.04 & IRAS 16362-4845 & 1 \\
\hline $338.37+0.16$ & 250.03 & -46.38 & $\ldots$ & $\ldots$ & $\ldots$ & $\ldots$ & 3.0 & 2.1 & 13.31 & 0.24 & $\ldots$ & $\ldots$ & IRAS 16330-4609 & 0 \\
\hline $338.90+0.58$ & 250.09 & -45.69 & 3.1 & 2.8 & 9.75 & 0.21 & 2.9 & 2.0 & 13.84 & 0.22 & 0.76 & 0.06 & IRAS 16364-4535 & 0 \\
\hline $338.41+0.08$ & 250.17 & -46.40 & 5.9 & 3.8 & 41.82 & 0.29 & 3.5 & 2.8 & 33.13 & 0.30 & -0.51 & 0.02 & IRAS 16371-4617 & 1 \\
\hline $338.12-0.18$ & 250.17 & -46.79 & $\ldots$ & $\ldots$ & $\ldots$ & $\ldots$ & 2.4 & 1.9 & 4.16 & 0.28 & $\ldots$ & $\ldots$ & IRAS 16331-4637 & 0 \\
\hline $337.92-0.48$ & 250.30 & -47.13 & 2.6 & 2.3 & 14.92 & 0.14 & 2.1 & 1.7 & 18.88 & 0.16 & 0.51 & 0.03 & IRAS 16374-4701 & 1 \\
\hline $338.91+0.37$ & 250.32 & -45.83 & 3.7 & 2.3 & 2.39 & 0.25 & 2.3 & 1.7 & 3.22 & 0.20 & 0.65 & 0.26 & IRAS 16377-4545 & 0 \\
\hline $336.55-1.80$ & 250.43 & -49.03 & 4.1 & 3.0 & 1.80 & 0.31 & 6.4 & 3.0 & 4.16 & 1.13 & 1.82 & 0.70 & IRAS 16379-4856 & 1 \\
\hline $338.42-0.22$ & 250.50 & -46.59 & 3.1 & 2.1 & 3.26 & 0.16 & 3.4 & 1.5 & 4.80 & 0.20 & 0.84 & 0.14 & IRAS 16381-4629 & 1 \\
\hline $337.50-1.08$ & 250.56 & -47.84 & $\ldots$ & $\ldots$ & $\ldots$ & $\ldots$ & 2.2 & 2.0 & 0.86 & 0.39 & $\ldots$ & $\ldots$ & IRAS $16350-4738$ & 0 \\
\hline $338.56-0.16$ & 250.56 & -46.44 & $\ldots$ & $\ldots$ & $\ldots$ & $\ldots$ & 2.2 & 1.7 & 0.93 & 0.31 & $\ldots$ & $\ldots$ & none & 0 \\
\hline $337.57-1.06$ & 250.61 & -47.78 & 5.5 & 5.2 & 2.69 & 2.75 & $\ldots$ & $\ldots$ & $\ldots$ & $\ldots$ & $\ldots$ & $\ldots$ & IRAS 16387-4741 & 0 \\
\hline $338.93-0.07$ & 250.82 & -46.10 & 2.7 & 2.5 & 3.46 & 0.16 & 2.3 & 2.0 & 3.79 & 0.21 & 0.19 & 0.16 & IRAS $16395-4600$ & 0 \\
\hline $339.28+0.22$ & 250.84 & -45.65 & 5.5 & 2.5 & 2.97 & 0.36 & 3.6 & 1.4 & 1.57 & 0.40 & -1.39 & 0.61 & IRAS 16397-4532 & 1 \\
\hline $339.20+0.12$ & 250.87 & -45.78 & $\ldots$ & $\ldots$ & $\ldots$ & $\ldots$ & 6.0 & 3.6 & 6.57 & 44.55 & $\ldots$ & $\ldots$ & IRAS 16364-4535 & 0 \\
\hline $339.10-0.22$ & 251.14 & -46.07 & 4.8 & 3.0 & 3.27 & 0.35 & 4.4 & 2.1 & 4.64 & 0.39 & 0.77 & 0.30 & none & 0 \\
\hline $339.49+0.07$ & 251.19 & -45.59 & 4.9 & 2.3 & 1.48 & 0.45 & 2.4 & 1.9 & 1.92 & 0.31 & 0.57 & 0.75 & IRAS $16410-4529$ & 1 \\
\hline
\end{tabular}


Table 2

(Continued)

\begin{tabular}{|c|c|c|c|c|c|c|c|c|c|c|c|c|c|c|}
\hline \multirow[t]{2}{*}{ Source } & \multirow[b]{2}{*}{$\begin{array}{l}\text { R.A. } \\
\text { (deg) }\end{array}$} & \multirow[b]{2}{*}{$\begin{array}{l}\text { Decl. } \\
\text { (deg) }\end{array}$} & \multicolumn{4}{|c|}{$100 \mathrm{GHz}$} & \multicolumn{4}{|c|}{$150 \mathrm{GHz}$} & \multirow[t]{2}{*}{$\alpha_{I}$} & \multirow[t]{2}{*}{$\delta \alpha_{I}$} & \multirow[t]{2}{*}{ Alt. Name } & \multirow[t]{2}{*}{ WC } \\
\hline & & & $\begin{array}{c}\sigma_{\text {maj }} \\
(\operatorname{arcmin})\end{array}$ & $\begin{array}{c}\sigma_{\min } \\
(\operatorname{arcmin})\end{array}$ & $\begin{array}{c}I \\
(\mathrm{Jy})\end{array}$ & $\begin{array}{l}\delta I \\
(\mathrm{Jy})\end{array}$ & $\begin{array}{c}\sigma_{\text {maj }} \\
(\operatorname{arcmin})\end{array}$ & $\begin{array}{c}\sigma_{\min } \\
(\operatorname{arcmin})\end{array}$ & $\begin{array}{c}I \\
(\mathrm{Jy})\end{array}$ & $\begin{array}{l}\delta I \\
(\mathrm{Jy})\end{array}$ & & & & \\
\hline $339.56+0.07$ & 251.27 & -45.53 & 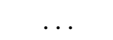 & & & & 2.7 & 2.0 & 2.09 & 0.31 & & & none & 0 \\
\hline $339.83+0.28$ & 251.29 & -45.19 & 2.4 & 2.2 & 1.32 & 0.18 & 2.2 & 1.8 & 2.17 & 0.24 & 1.08 & 0.38 & none & 0 \\
\hline $339.14-0.37$ & 251.34 & -46.14 & 6.1 & 3.7 & 3.83 & 1.04 & $\ldots$ & $\ldots$ & $\ldots$ & $\ldots$ & $\ldots$ & $\ldots$ & IRAS 16419-4602 & 0 \\
\hline $340.24+0.50$ & 251.42 & -44.74 & 6.8 & 2.6 & 3.87 & 0.56 & $\ldots$ & $\ldots$ & $\ldots$ & $\ldots$ & $\ldots$ & $\ldots$ & IRAS $16420-4440$ & 0 \\
\hline $339.58-0.12$ & 251.49 & -45.64 & 3.1 & 2.6 & 3.60 & 0.20 & 2.8 & 2.2 & 6.30 & 0.24 & 1.22 & 0.15 & IRAS 16421-4532 & 1 \\
\hline $339.28-0.41$ & 251.53 & -46.06 & $\ldots$ & $\ldots$ & $\ldots$ & & 8.6 & 2.8 & 5.33 & 3.52 & $\ldots$ & $\ldots$ & IRAS 16386-4553 & 0 \\
\hline $339.04-0.64$ & 251.54 & -46.39 & 10.2 & 1.8 & 1.70 & 3.33 & $\ldots$ & $\ldots$ & $\ldots$ & $\ldots$ & $\ldots$ & $\ldots$ & none & 0 \\
\hline $339.00-0.72$ & 251.60 & -46.47 & & $\ldots$ & s. & & 4.6 & 2.0 & 1.71 & 1.05 & $\ldots$ & 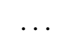 & IRAS 16389-4614 & 0 \\
\hline $343.07+2.58$ & 251.77 & -41.24 & 2.8 & 1.6 & 0.81 & 0.24 & 3.3 & 1.6 & 3.01 & 0.30 & 2.86 & 0.68 & IRAS $16435-4110$ & 0 \\
\hline $340.06-0.23$ & 252.04 & -45.35 & 4.6 & 2.9 & 4.27 & 0.29 & 3.4 & 1.5 & 3.11 & 0.37 & -0.69 & 0.30 & IRAS $16445-4516$ & 1 \\
\hline $340.25-0.07$ & 252.05 & -45.10 & $\ldots$ & $\ldots$ & $\ldots$ & $\ldots$ & 3.4 & 2.1 & 3.26 & 0.49 & $\ldots$ & $\ldots$ & IRAS 16407-4453 & 0 \\
\hline $340.27-0.22$ & 252.22 & -45.18 & 4.6 & 3.4 & 8.63 & 0.43 & 2.6 & 2.2 & 9.03 & 0.25 & 0.10 & 0.12 & IRAS 16452-4504 & 1 \\
\hline $339.95-0.56$ & 252.31 & -45.65 & 2.7 & 2.1 & 1.51 & 0.18 & 2.2 & 1.8 & 2.28 & 0.22 & 0.90 & 0.34 & IRAS 16455-4531 & 1 \\
\hline $340.52-0.15$ & 252.38 & -44.94 & $\ldots$ & $\ldots$ & $\ldots$ & $\ldots$ & 2.0 & 1.6 & 0.86 & 0.48 & $\ldots$ & $\ldots$ & none & 0 \\
\hline $340.24-0.39$ & 252.39 & -45.31 & $\ldots$ & $\ldots$ & $\ldots$ & $\ldots$ & 2.0 & 1.8 & 3.17 & 0.20 & $\ldots$ & $\ldots$ & none & 0 \\
\hline $341.27+0.35$ & 252.51 & -44.05 & $\ldots$ & $\ldots$ & $\ldots$ & $\ldots$ & 4.4 & 1.8 & 2.42 & 0.95 & $\ldots$ & $\ldots$ & IRAS 16431-4353 & 0 \\
\hline $340.37-0.40$ & 252.52 & -45.22 & $\ldots$ & $\ldots$ & $\ldots$ & $\ldots$ & 2.2 & 1.5 & 1.64 & 0.24 & & $\ldots$ & IRAS 16430-4501 & 0 \\
\hline $340.80-0.11$ & 252.59 & -44.71 & $\ldots$ & $\ldots$ & $\ldots$ & $\ldots$ & 3.7 & 2.5 & 2.71 & 0.98 & $\ldots$ & $\ldots$ & IRAS $16433-4432$ & 0 \\
\hline $341.02-0.09$ & 252.77 & -44.52 & 5.8 & 3.3 & 2.08 & 0.75 & 3.4 & 2.4 & 2.22 & 0.56 & 0.13 & 0.96 & IRAS $16473-4425$ & 0 \\
\hline $339.69-1.20$ & 252.78 & -46.25 & 3.8 & 2.4 & 1.60 & 0.33 & 3.7 & 1.7 & 4.24 & 0.27 & 2.12 & 0.47 & IRAS $16474-4610$ & 1 \\
\hline $341.29+0.12$ & 252.78 & -44.17 & $\ldots$ & $\ldots$ & $\ldots$ & $\ldots$ & 6.6 & 2.1 & 3.53 & 1.22 & $\ldots$ & $\ldots$ & none & 0 \\
\hline $341.24+0.05$ & 252.81 & -44.27 & 3.2 & 1.6 & 0.88 & 2.41 & $\ldots$ & $\ldots$ & $\ldots$ & $\ldots$ & $\ldots$ & $\ldots$ & IRAS $16475-4410$ & 0 \\
\hline $340.91-0.25$ & 252.84 & -44.71 & $\ldots$ & $\ldots$ & $\ldots$ & $\ldots$ & 3.3 & 1.7 & 1.64 & 1.21 & $\ldots$ & $\ldots$ & IRAS 16442-4435 & 0 \\
\hline $340.07-1.08$ & 253.00 & -45.89 & $\ldots$ & $\ldots$ & $\ldots$ & $\ldots$ & 3.2 & 1.6 & 1.45 & 0.42 & $\ldots$ & $\ldots$ & none & 0 \\
\hline $339.88-1.26$ & 253.02 & -46.15 & 3.0 & 1.9 & 1.13 & 0.29 & 1.9 & 1.7 & 3.41 & 0.16 & 2.41 & 0.56 & IRAS 16484-4603 & 1 \\
\hline $340.99-0.38$ & 253.06 & -44.73 & $\ldots$ & $\ldots$ & $\ldots$ & $\ldots$ & 5.0 & 2.5 & 3.46 & 1.67 & $\ldots$ & $\ldots$ & IRAS 16449-4430 & 0 \\
\hline $342.06+0.42$ & 253.14 & -43.40 & 2.9 & 2.2 & 3.25 & 0.19 & 2.2 & 1.7 & 4.27 & 0.22 & 0.59 & 0.17 & IRAS 16489-4318 & 1 \\
\hline $341.13-0.37$ & 253.17 & -44.62 & $\ldots$ & $\ldots$ & $\ldots$ & $\ldots$ & 2.8 & 2.0 & 1.84 & 0.36 & $\ldots$ & $\ldots$ & none & 0 \\
\hline $341.71+0.09$ & 253.19 & -43.88 & $\ldots$ & $\ldots$ & $\ldots$ & $\ldots$ & 4.6 & 3.0 & 6.50 & 0.60 & $\ldots$ & $\ldots$ & none & 0 \\
\hline $341.24-0.32$ & 253.21 & -44.50 & 5.4 & 5.0 & 7.55 & 0.53 & 4.5 & 2.1 & 8.21 & 0.41 & 0.18 & 0.19 & IRAS 16491-4427 & 0 \\
\hline $341.70+0.01$ & 253.26 & -43.93 & 10.5 & 3.9 & 5.07 & 4.73 & $\ldots$ & $\ldots$ & $\ldots$ & $\ldots$ & $\ldots$ & $\ldots$ & IRAS 16492-4349 & 1 \\
\hline $341.97+0.21$ & 253.28 & -43.60 & 4.1 & 2.2 & 1.48 & 0.30 & 4.5 & 2.1 & 2.77 & 0.83 & 1.37 & 0.79 & IRAS 16494-4330 & 1 \\
\hline $342.28+0.30$ & 253.46 & -43.30 & 3.9 & 3.6 & 4.35 & 0.31 & 5.6 & 3.3 & 6.39 & 0.96 & 0.84 & 0.36 & IRAS 16501-4314 & 0 \\
\hline $342.10+0.04$ & 253.58 & -43.60 & 5.0 & 2.3 & 1.50 & 0.85 & $\ldots$ & $\ldots$ & $\ldots$ & $\ldots$ & $\ldots$ & $\ldots$ & IRAS 16507-4333 & 0 \\
\hline $340.80-1.03$ & 253.60 & -45.29 & 3.6 & 2.5 & 12.83 & 0.21 & 3.0 & 1.9 & 14.53 & 0.25 & 0.27 & 0.05 & IRAS 16506-4512 & 1 \\
\hline $341.94-0.18$ & 253.68 & -43.87 & $\ldots$ & $\ldots$ & $\ldots$ & $\ldots$ & 1.9 & 1.7 & 2.10 & 0.23 & $\ldots$ & $\ldots$ & none & 0 \\
\hline $342.37+0.09$ & 253.76 & -43.36 & 3.5 & 2.3 & 1.40 & 0.28 & 3.7 & 1.9 & 3.65 & 0.39 & 2.09 & 0.50 & IRAS $16513-4316 \mathrm{~A}$ & 1 \\
\hline $342.49+0.17$ & 253.78 & -43.21 & $\ldots$ & $\ldots$ & $\ldots$ & $\ldots$ & 2.4 & 2.1 & 2.15 & 0.31 & $\ldots$ & $\ldots$ & IRAS 16479-4259 & 0 \\
\hline $342.36-0.04$ & 253.88 & -43.45 & 3.1 & 2.3 & 1.40 & 0.23 & 4.6 & 3.7 & 6.12 & 1.17 & 3.21 & 0.55 & IRAS $16520-4322$ & 0 \\
\hline $342.70+0.12$ & 254.01 & -43.09 & $\ldots$ & $\ldots$ & $\ldots$ & $\ldots$ & 2.4 & 1.7 & 2.85 & 0.27 & $\ldots$ & $\ldots$ & none & 0 \\
\hline $341.99-0.50$ & 254.07 & -44.03 & $\ldots$ & $\ldots$ & $\ldots$ & $\ldots$ & 2.6 & 1.9 & 1.99 & 13.91 & $\ldots$ & $\ldots$ & IRAS $16488-4353$ & 0 \\
\hline $344.99+1.79$ & 254.18 & -40.26 & 3.5 & 2.5 & 5.16 & 0.27 & 1.9 & 1.8 & 12.20 & 0.17 & 1.87 & 0.12 & IRAS 16533-4009 & 1 \\
\hline $342.03-0.69$ & 254.31 & -44.12 & $\ldots$ & $\ldots$ & $\ldots$ & $\cdots$ & 8.4 & 0.8 & 8.83 & 46.83 & $\ldots$ & $\ldots$ & IRAS 16500-4359 & 0 \\
\hline $345.08+1.59$ & 254.47 & -40.31 & $\ldots$ & $\ldots$ & $\ldots$ & $\ldots$ & 8.2 & 3.1 & 8.97 & 1.31 & $\ldots$ & $\ldots$ & none & 0 \\
\hline $345.01+1.53$ & 254.47 & -40.40 & 8.5 & 4.5 & 11.16 & 0.63 & $\ldots$ & $\ldots$ & $\ldots$ & $\ldots$ & $\ldots$ & $\ldots$ & IRAS 16544-4019 & 0 \\
\hline $343.14-0.05$ & 254.57 & -42.86 & 3.0 & 2.4 & 1.25 & 0.24 & 1.9 & 1.6 & 3.54 & 0.18 & 2.27 & 0.44 & IRAS 16547-4247 & 1 \\
\hline $344.96+1.23$ & 254.73 & -40.63 & $\ldots$ & $\ldots$ & $\ldots$ & $\ldots$ & 13.7 & 2.3 & 10.73 & 1.80 & $\ldots$ & $\ldots$ & IRAS $16517-4030$ & 0 \\
\hline $343.47-0.04$ & 254.84 & -42.58 & 3.0 & 2.8 & 9.51 & 0.21 & 2.5 & 2.0 & 9.32 & 0.23 & -0.04 & 0.07 & IRAS 16558-4228 & 1 \\
\hline $345.79+1.77$ & 254.85 & -39.64 & $\ldots$ & $\ldots$ & $\ldots$ & $\ldots$ & 2.6 & 1.4 & 0.89 & 0.29 & $\ldots$ & $\ldots$ & IRAS 16525-3931 & 0 \\
\hline $345.37+1.42$ & 254.88 & -40.19 & 4.8 & 3.1 & 24.35 & 0.28 & 3.8 & 2.1 & 24.82 & 0.27 & 0.04 & 0.03 & IRAS 16561-4006 & 1 \\
\hline $345.49+1.46$ & 254.93 & -40.06 & $\ldots$ & $\ldots$ & $\ldots$ & $\ldots$ & 2.8 & 1.6 & 11.19 & 0.23 & $\ldots$ & $\ldots$ & none & 0 \\
\hline $345.22+1.03$ & 255.16 & -40.55 & 3.1 & 2.5 & 10.94 & 0.20 & 2.6 & 1.9 & 15.51 & 0.25 & 0.76 & 0.05 & IRAS 16571-4029 & 1 \\
\hline $344.00+0.05$ & 255.18 & -42.11 & $\ldots$ & $\ldots$ & $\ldots$ & $\ldots$ & 8.8 & 3.9 & 8.17 & 19.59 & $\ldots$ & $\ldots$ & IRAS 16537-4155 & 0 \\
\hline $343.75-0.17$ & 255.21 & -42.45 & $\ldots$ & $\ldots$ & $\ldots$ & $\cdots$ & 3.9 & 2.0 & 5.23 & 0.47 & $\ldots$ & $\ldots$ & none & 0 \\
\hline $343.51-0.54$ & 255.41 & -42.86 & $\cdots$ & $\ldots$ & $\ldots$ & $\ldots$ & 3.0 & 1.8 & 1.90 & 0.36 & $\ldots$ & $\ldots$ & IRAS 16544-4241 & 0 \\
\hline $345.74+1.14$ & 255.46 & -40.07 & $\ldots$ & $\ldots$ & $\ldots$ & $\ldots$ & 3.1 & 1.5 & 0.90 & 0.49 & $\ldots$ & $\ldots$ & IRAS 16549-3951 & 0 \\
\hline $344.42+0.04$ & 255.54 & -41.78 & 2.4 & 2.1 & 2.37 & 0.15 & 1.8 & 1.5 & 3.63 & 0.20 & 0.93 & 0.18 & IRAS 16586-4142 & 1 \\
\hline $345.71+0.81$ & 255.78 & -40.29 & $\ldots$ & $\ldots$ & $\ldots$ & $\ldots$ & 1.7 & 1.4 & 1.12 & 0.17 & $\ldots$ & $\ldots$ & IRAS 16561-4006 & 1 \\
\hline $343.90-0.64$ & 255.84 & -42.61 & 2.5 & 1.9 & 0.62 & 0.30 & 2.5 & 1.2 & 0.99 & 0.78 & 1.03 & 2.02 & IRAS 16598-4234 & 0 \\
\hline $344.09-0.65$ & 256.01 & -42.47 & $\ldots$ & $\ldots$ & $\ldots$ & $\ldots$ & 2.3 & 2.0 & 2.88 & 0.27 & $\ldots$ & $\ldots$ & PMN J1704-4228 & 0 \\
\hline $344.22-0.59$ & 256.05 & -42.33 & 2.7 & 2.2 & 3.11 & 0.18 & 2.3 & 1.6 & 7.99 & 0.21 & 2.05 & 0.14 & IRAS 17006-4215 & 1 \\
\hline $345.49+0.32$ & 256.11 & -40.77 & 3.0 & 2.2 & 7.36 & 0.18 & 2.3 & 1.5 & 12.88 & 0.18 & 1.22 & 0.06 & IRAS $17009-4042$ & 1 \\
\hline $345.04-0.22$ & 256.32 & -41.45 & 6.0 & 2.5 & 5.35 & 0.32 & 3.6 & 1.9 & 11.57 & 0.40 & 1.68 & 0.15 & IRAS 17016-4124 & 1 \\
\hline $346.83+0.95$ & 256.51 & -39.32 & $\ldots$ & $\ldots$ & $\ldots$ & $\ldots$ & 3.5 & 1.9 & 1.13 & 1.01 & $\ldots$ & $\ldots$ & none & 0 \\
\hline
\end{tabular}


Table 2

(Continued)

\begin{tabular}{|c|c|c|c|c|c|c|c|c|c|c|c|c|c|c|}
\hline \multirow[t]{2}{*}{ Source } & \multirow[b]{2}{*}{$\begin{array}{l}\text { R.A. } \\
\text { (deg) }\end{array}$} & \multirow[b]{2}{*}{$\begin{array}{l}\text { Decl. } \\
\text { (deg) }\end{array}$} & \multicolumn{4}{|c|}{$100 \mathrm{GHz}$} & \multicolumn{4}{|c|}{$150 \mathrm{GHz}$} & \multirow[t]{2}{*}{$\alpha_{I}$} & \multirow[t]{2}{*}{$\delta \alpha_{I}$} & \multirow[t]{2}{*}{ Alt. Name } & \multirow[t]{2}{*}{ WC } \\
\hline & & & $\begin{array}{c}\sigma_{\text {maj }} \\
(\operatorname{arcmin})\end{array}$ & $\begin{array}{c}\sigma_{\min } \\
(\operatorname{arcmin})\end{array}$ & $\begin{array}{c}I \\
(\mathrm{Jy})\end{array}$ & $\begin{array}{c}\delta I \\
(\mathrm{Jy})\end{array}$ & $\begin{array}{c}\sigma_{\text {maj }} \\
(\operatorname{arcmin})\end{array}$ & $\begin{array}{c}\sigma_{\min } \\
(\operatorname{arcmin})\end{array}$ & $\begin{array}{c}I \\
(\mathrm{Jy})\end{array}$ & $\begin{array}{l}\delta I \\
(\mathrm{Jy})\end{array}$ & & & & \\
\hline $345.59-0.03$ & 256.56 & -40.90 & 5.2 & 2.6 & 12.09 & 0.25 & 5.0 & 2.0 & 15.09 & 0.34 & 0.48 & 0.07 & IRAS $17028-4050$ & 1 \\
\hline $347.77+1.50$ & 256.68 & -38.24 & $\ldots$ & $\ldots$ & $\ldots$ & $\ldots$ & 3.8 & 2.3 & 2.15 & 0.47 & $\ldots$ & $\ldots$ & none & 0 \\
\hline $345.84+0.02$ & 256.71 & -40.68 & 5.2 & 2.9 & 2.42 & 0.68 & 7.1 & 2.4 & 6.03 & 0.73 & 1.99 & 0.67 & IRAS 17033-4035 & 0 \\
\hline $346.12-0.07$ & 257.02 & -40.50 & 6.6 & 3.3 & 5.90 & 0.38 & 6.5 & 4.8 & 9.80 & 1.00 & 1.10 & 0.26 & IRAS $17044-4025$ & 0 \\
\hline $345.20-0.78$ & 257.05 & -41.67 & 4.5 & 3.3 & 3.44 & 0.37 & 3.4 & 1.1 & 1.55 & 0.60 & -1.74 & 0.88 & IRAS 17044-4134 & 1 \\
\hline $345.15-0.86$ & 257.09 & -41.75 & $\ldots$ & $\ldots$ & $\ldots$ & $\ldots$ & 3.1 & 1.6 & 1.64 & 0.54 & $\ldots$ & $\ldots$ & IRAS $17012-4138$ & 0 \\
\hline $346.53+0.08$ & 257.18 & -40.08 & 2.6 & 2.2 & 1.37 & 0.17 & 2.2 & 1.9 & 2.79 & 0.22 & 1.54 & 0.32 & IRAS 17052-4001 & 1 \\
\hline $345.40-0.95$ & 257.39 & -41.60 & 2.4 & 2.3 & 23.41 & 0.16 & 1.7 & 1.7 & 25.23 & 0.19 & 0.16 & 0.02 & IRAS 17059-4132 & 1 \\
\hline $346.83+0.06$ & 257.45 & -39.85 & $\ldots$ & $\ldots$ & $\ldots$ & $\ldots$ & 6.7 & 2.5 & 3.28 & 2.65 & $\ldots$ & $\ldots$ & none & 0 \\
\hline $347.39+0.27$ & 257.66 & -39.28 & 4.1 & 2.1 & 1.79 & 0.23 & 3.0 & 1.6 & 1.61 & 0.26 & -0.23 & 0.45 & IRAS 17070-3914 & 0 \\
\hline $347.29+0.15$ & 257.71 & -39.43 & 2.9 & 1.8 & 0.57 & 0.26 & 2.1 & 1.9 & 1.17 & 0.21 & 1.57 & 1.06 & IRAS 17074-3922 & 0 \\
\hline $347.19-0.01$ & 257.80 & -39.61 & 7.1 & 2.9 & 3.01 & 0.46 & 3.5 & 1.9 & 2.79 & 0.25 & -0.17 & 0.39 & IRAS $17075-3933$ & 0 \\
\hline $342.12-3.73$ & 257.80 & -45.89 & $\ldots$ & $\ldots$ & $\ldots$ & $\ldots$ & 2.0 & 1.6 & 1.84 & 0.36 & $\ldots$ & $\ldots$ & IRAS $17037-4545$ & 0 \\
\hline $347.14-0.09$ & 257.84 & -39.69 & $\ldots$ & $\ldots$ & $\ldots$ & $\ldots$ & 3.9 & 2.6 & 2.45 & 0.57 & $\ldots$ & $\ldots$ & none & 0 \\
\hline $346.39-0.65$ & 257.85 & -40.63 & $\ldots$ & $\ldots$ & $\ldots$ & $\ldots$ & 3.5 & 2.1 & 1.98 & 0.49 & $\ldots$ & 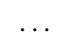 & IRAS $17043-4027$ & 1 \\
\hline $347.61+0.19$ & 257.91 & -39.14 & 3.8 & 2.8 & 12.58 & 0.19 & 3.5 & 2.3 & 15.91 & 0.23 & 0.51 & 0.05 & IRAS 17079-3905 & 1 \\
\hline $345.89-1.11$ & 257.94 & -41.31 & $\ldots$ & $\ldots$ & $\ldots$ & $\ldots$ & 1.8 & 1.2 & 1.24 & 0.17 & $\ldots$ & $\ldots$ & none & 0 \\
\hline $348.22+0.46$ & 258.08 & -38.50 & 3.5 & 2.9 & 7.19 & 0.19 & 3.0 & 2.2 & 9.52 & 0.26 & 0.61 & 0.08 & none & 0 \\
\hline $347.89+0.03$ & 258.28 & -39.01 & 3.3 & 2.0 & 2.57 & 0.16 & 3.9 & 1.8 & 4.91 & 0.24 & 1.41 & 0.17 & IRAS 17096-3856 & 1 \\
\hline $349.50+1.05$ & 258.43 & -37.11 & 2.2 & 1.9 & 1.78 & 0.12 & 1.5 & 1.4 & 1.64 & 0.12 & -0.18 & 0.22 & IRAS 17103-3702 & 1 \\
\hline $348.68+0.38$ & 258.52 & -38.17 & 12.4 & 7.3 & 9.43 & 2.06 & $\ldots$ & $\ldots$ & $\ldots$ & $\ldots$ & $\ldots$ & $\ldots$ & IRAS $17109-3807$ & 0 \\
\hline $348.42+0.11$ & 258.60 & -38.54 & 5.3 & 3.9 & 6.75 & 0.30 & 8.2 & 6.9 & 13.40 & 1.97 & 1.49 & 0.33 & IRAS $17107-3829$ & 0 \\
\hline $347.87-0.31$ & 258.63 & -39.23 & $\ldots$ & $\ldots$ & $\ldots$ & $\ldots$ & 2.7 & 1.7 & 1.68 & 0.22 & $\ldots$ & $\ldots$ & none & 0 \\
\hline $350.38+1.35$ & 258.77 & -36.22 & $\ldots$ & $\ldots$ & $\ldots$ & $\ldots$ & 4.1 & 2.5 & 1.69 & 1.43 & $\ldots$ & $\ldots$ & IRAS 17084-3605 & 0 \\
\hline $348.76+0.17$ & 258.80 & -38.23 & $\ldots$ & $\ldots$ & $\ldots$ & $\ldots$ & 11.7 & 4.4 & 8.80 & 3.94 & $\ldots$ & $\ldots$ & IRAS 17084-3809 & 0 \\
\hline $347.97-0.45$ & 258.85 & -39.23 & 3.2 & 2.3 & 2.09 & 0.23 & 2.0 & 1.7 & 2.55 & 0.15 & 0.44 & 0.28 & IRAS 17118-3909 & 1 \\
\hline $349.13+0.04$ & 259.19 & -38.00 & 4.2 & 3.7 & 10.38 & 0.24 & 4.7 & 3.4 & 18.02 & 0.36 & 1.20 & 0.07 & IRAS 17135-3755 & 1 \\
\hline $348.88-0.19$ & 259.26 & -38.34 & $\ldots$ & $\ldots$ & $\ldots$ & $\ldots$ & 3.2 & 2.1 & 1.67 & 0.35 & $\ldots$ & $\ldots$ & IRAS $17102-3813$ & 1 \\
\hline $350.52+0.96$ & 259.27 & -36.34 & 2.9 & 2.3 & 5.53 & 0.16 & 1.9 & 1.5 & 6.50 & 0.13 & 0.35 & 0.08 & IRAS $17136-3617$ & 1 \\
\hline $352.74+2.39$ & 259.40 & -33.69 & 2.4 & 1.7 & 1.17 & 0.16 & $\ldots$ & $\ldots$ & $\ldots$ & $\ldots$ & $\ldots$ & $\ldots$ & IRAS $17141-3337$ & 0 \\
\hline $350.74+0.93$ & 259.45 & -36.17 & $\ldots$ & $\ldots$ & $\ldots$ & $\ldots$ & 2.6 & 2.1 & 3.19 & 0.22 & $\ldots$ & $\ldots$ & none & 0 \\
\hline $351.98+1.81$ & 259.45 & -34.66 & $\ldots$ & $\ldots$ & $\ldots$ & $\ldots$ & 2.7 & 1.5 & 0.93 & 0.48 & $\ldots$ & $\ldots$ & IRAS 17114-3433 & 0 \\
\hline $348.60-0.58$ & 259.45 & -38.79 & 8.7 & 3.0 & 6.20 & 0.37 & 9.9 & 3.8 & 7.86 & 1.51 & 0.52 & 0.44 & none & 0 \\
\hline $349.73+0.16$ & 259.51 & -37.45 & 11.2 & 2.9 & 6.61 & 0.63 & 2.2 & 1.8 & 1.88 & 0.21 & -2.73 & 0.32 & IRAS $17146-3723$ & 1 \\
\hline $348.24-0.98$ & 259.61 & -39.32 & 2.3 & 2.2 & 7.37 & 0.13 & 1.8 & 1.7 & 8.10 & 0.14 & 0.21 & 0.05 & IRAS 17149-3916 & 1 \\
\hline $349.85+0.08$ & 259.68 & -37.39 & $\ldots$ & $\ldots$ & $\ldots$ & $\ldots$ & 5.6 & 3.4 & 5.45 & 0.68 & $\ldots$ & $\ldots$ & none & 0 \\
\hline $351.28+1.04$ & 259.73 & -35.66 & $\ldots$ & $\ldots$ & $\ldots$ & $\ldots$ & 3.0 & 1.7 & 2.05 & 0.32 & $\ldots$ & $\ldots$ & IRAS $17122-3533$ & 0 \\
\hline $348.54-0.98$ & 259.83 & -39.08 & $\ldots$ & $\ldots$ & $\ldots$ & $\ldots$ & 2.0 & 1.3 & 3.44 & 0.13 & $\ldots$ & $\ldots$ & IRAS $17123-3856$ & 0 \\
\hline $350.12+0.08$ & 259.88 & -37.18 & 2.6 & 2.4 & 6.86 & 0.14 & 2.1 & 1.9 & 11.15 & 0.18 & 1.06 & 0.06 & IRAS 17160-3707 & 1 \\
\hline $348.91-0.79$ & 259.90 & -38.67 & $\ldots$ & $\ldots$ & $\ldots$ & $\ldots$ & 3.8 & 1.7 & 1.28 & 0.37 & $\ldots$ & $\ldots$ & IRAS 17127-3834 & 0 \\
\hline $351.04+0.66$ & 259.95 & -36.08 & 3.4 & 2.8 & 19.32 & 0.20 & 3.2 & 2.1 & 9.39 & 0.24 & -1.57 & 0.06 & IRAS $17163-3603$ & 1 \\
\hline $350.33+0.14$ & 259.97 & -36.96 & 3.5 & 2.1 & 1.53 & 0.22 & 2.6 & 1.7 & 2.72 & 0.18 & 1.25 & 0.35 & IRAS $17164-3653$ & 1 \\
\hline $348.72-1.04$ & 260.03 & -38.96 & 2.7 & 2.4 & 29.34 & 0.15 & 2.0 & 1.8 & 29.89 & 0.15 & 0.04 & 0.02 & IRAS $17167-3854$ & 1 \\
\hline $351.34+0.68$ & 260.13 & -35.82 & 5.7 & 3.1 & 117.79 & 0.25 & 6.2 & 2.3 & 144.62 & 0.34 & 0.45 & 0.01 & IRAS $17172-3548$ & 0 \\
\hline $351.19+0.49$ & 260.22 & -36.05 & 2.7 & 2.3 & 8.17 & 0.17 & 2.4 & 1.6 & 5.85 & 0.17 & -0.73 & 0.08 & IRAS $17175-3558$ & 0 \\
\hline $349.12-1.00$ & 260.27 & -38.61 & $\ldots$ & $\ldots$ & $\ldots$ & $\ldots$ & 2.3 & 2.1 & 1.03 & 0.32 & $\ldots$ & $\ldots$ & IRAS $17143-3832$ & 0 \\
\hline $349.83-0.55$ & 260.32 & -37.77 & 3.0 & 2.7 & 6.29 & 0.18 & 2.2 & 1.8 & 5.09 & 0.16 & -0.46 & 0.09 & IRAS $17178-3742$ & 1 \\
\hline $351.69+0.66$ & 260.40 & -35.55 & $\ldots$ & $\ldots$ & $\ldots$ & $\ldots$ & 3.1 & 1.2 & 1.24 & 0.39 & $\ldots$ & $\ldots$ & IRAS $17147-3524$ & 0 \\
\hline $350.01-0.52$ & 260.42 & -37.60 & $\ldots$ & $\ldots$ & $\ldots$ & $\ldots$ & 2.9 & 1.9 & 2.87 & 0.20 & $\ldots$ & $\ldots$ & IRAS $17151-3728$ & 0 \\
\hline $350.79-0.03$ & 260.46 & -36.69 & 2.9 & 2.5 & 2.13 & 0.17 & 2.6 & 2.0 & 3.24 & 0.21 & 0.91 & 0.22 & IRAS $17184-3638$ & 1 \\
\hline $351.84+0.64$ & 260.53 & -35.44 & $\ldots$ & $\ldots$ & $\ldots$ & $\ldots$ & 2.3 & 2.2 & 2.37 & 0.24 & $\ldots$ & $\ldots$ & none & 0 \\
\hline $352.82+1.29$ & 260.55 & -34.26 & 3.1 & 2.0 & 1.22 & 0.18 & 2.6 & 2.4 & 1.48 & 0.63 & 0.42 & 0.98 & IRAS 17186-3413 & 0 \\
\hline $350.26-0.49$ & 260.57 & -37.38 & $\ldots$ & $\ldots$ & $\ldots$ & $\ldots$ & 6.3 & 2.5 & 2.99 & 1.91 & $\ldots$ & $\ldots$ & IRAS $17155-3716$ & 0 \\
\hline $352.23+0.81$ & 260.63 & -35.02 & $\cdots$ & $\cdots$ & $\cdots$ & $\cdots$ & 2.1 & 1.4 & 0.81 & 0.26 & $\cdots$ & $\cdots$ & IRAS $17156-3456$ & 0 \\
\hline $350.53-0.37$ & 260.63 & -37.09 & $\ldots$ & $\ldots$ & $\ldots$ & $\ldots$ & 2.5 & 1.9 & 2.09 & 0.20 & $\ldots$ & $\ldots$ & IRAS 17155-3659 & 0 \\
\hline $352.10+0.72$ & 260.63 & -35.18 & $\ldots$ & $\ldots$ & $\ldots$ & $\ldots$ & 4.4 & 1.6 & 3.09 & 0.30 & $\ldots$ & $\ldots$ & none & 0 \\
\hline $350.77-0.29$ & 260.72 & -36.85 & $\ldots$ & $\ldots$ & $\ldots$ & $\ldots$ & 3.8 & 3.4 & 3.08 & 30.46 & $\ldots$ & $\ldots$ & none & 0 \\
\hline $349.63-1.11$ & 260.76 & -38.25 & $\ldots$ & $\ldots$ & $\ldots$ & $\ldots$ & 2.6 & 1.4 & 1.75 & 0.22 & $\ldots$ & $\ldots$ & IRAS $17163-3808$ & 0 \\
\hline $351.59+0.19$ & 260.81 & -35.90 & 3.2 & 2.3 & 11.41 & 0.15 & 3.0 & 1.7 & 12.50 & 0.18 & 0.20 & 0.04 & IRAS $17200-3550$ & 1 \\
\hline $352.50+0.80$ & 260.82 & -34.81 & $\ldots$ & $\ldots$ & $\ldots$ & $\ldots$ & 2.0 & 1.9 & 2.64 & 0.18 & $\ldots$ & $\ldots$ & none & 0 \\
\hline $350.69-0.49$ & 260.87 & -37.03 & $\ldots$ & $\ldots$ & $\ldots$ & $\ldots$ & 1.8 & 1.5 & 0.79 & 0.29 & $\ldots$ & $\ldots$ & IRAS 17166-3656 & 1 \\
\hline $351.04-0.33$ & 260.96 & -36.65 & $\ldots$ & $\ldots$ & $\ldots$ & $\ldots$ & 6.3 & 2.0 & 3.27 & 1.12 & $\cdots$ & $\cdots$ & IRAS $17169-3631$ & 0 \\
\hline $353.26+1.08$ & 261.05 & -34.02 & $\ldots$ & $\ldots$ & $\ldots$ & $\ldots$ & 2.5 & 2.1 & 2.58 & 0.29 & $\ldots$ & $\ldots$ & IRAS $17174-3355$ & 0 \\
\hline $353.01+0.91$ & 261.06 & -34.32 & $\ldots$ & $\ldots$ & $\ldots$ & $\ldots$ & 2.4 & 1.3 & 2.96 & 0.18 & $\ldots$ & $\ldots$ & none & 0 \\
\hline
\end{tabular}


Table 2

(Continued)

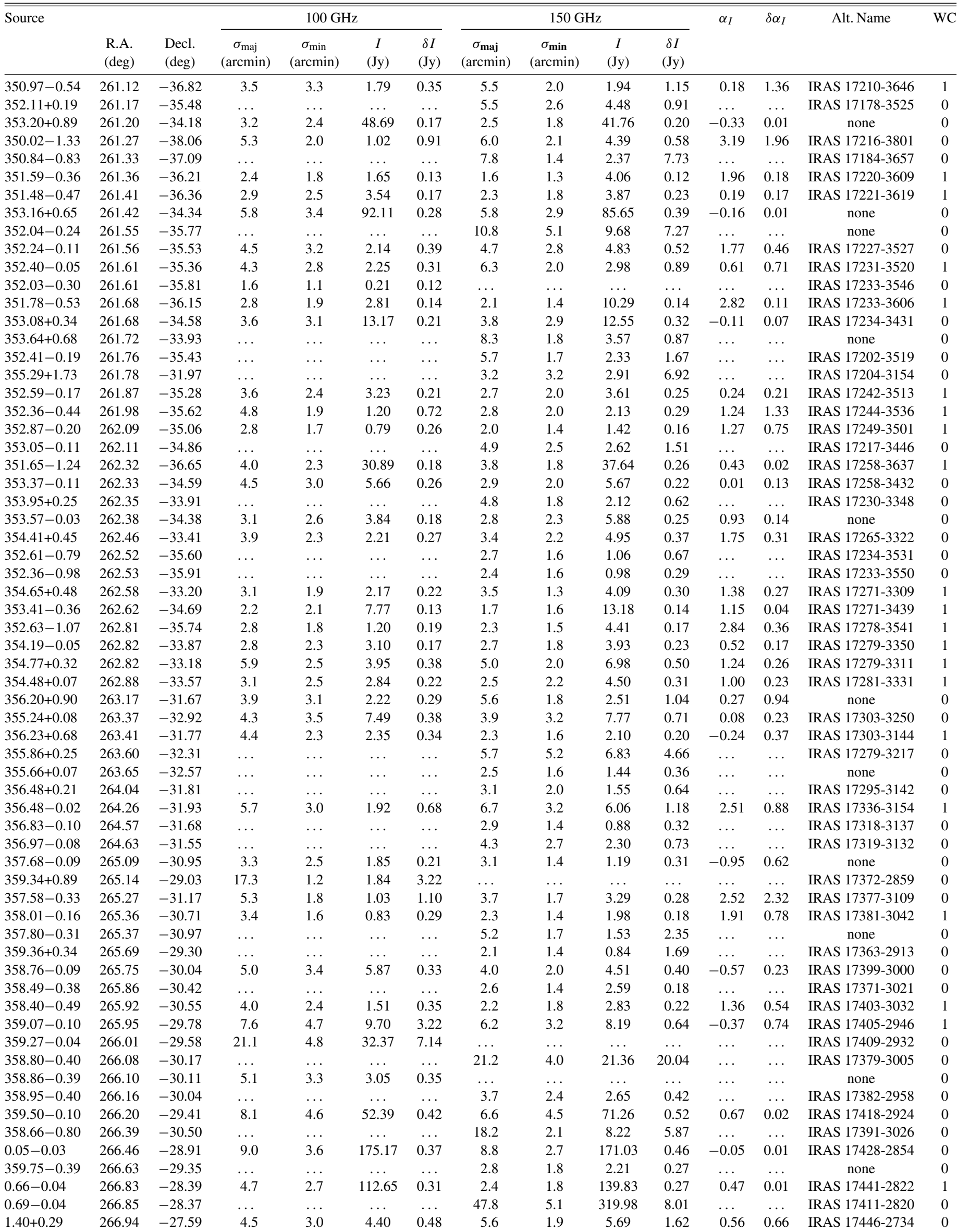


Table 2

(Continued)

\begin{tabular}{|c|c|c|c|c|c|c|c|c|c|c|c|c|c|c|}
\hline \multirow[t]{2}{*}{ Source } & \multirow[b]{2}{*}{$\begin{array}{l}\text { R.A. } \\
\text { (deg) }\end{array}$} & \multirow[b]{2}{*}{$\begin{array}{l}\text { Decl. } \\
\text { (deg) }\end{array}$} & \multicolumn{4}{|c|}{$100 \mathrm{GHz}$} & \multicolumn{4}{|c|}{$150 \mathrm{GHz}$} & \multirow[t]{2}{*}{$\alpha_{I}$} & \multirow[t]{2}{*}{$\delta \alpha_{I}$} & \multirow[t]{2}{*}{ Alt. Name } & \multirow[t]{2}{*}{ WC } \\
\hline & & & $\begin{array}{c}\sigma_{\mathrm{maj}} \\
(\operatorname{arcmin})\end{array}$ & $\begin{array}{c}\sigma_{\min } \\
(\operatorname{arcmin})\end{array}$ & $\begin{array}{c}I \\
(\mathrm{Jy})\end{array}$ & $\begin{array}{c}\delta I \\
(\mathrm{Jy})\end{array}$ & $\begin{array}{c}\sigma_{\text {maj }} \\
(\operatorname{arcmin})\end{array}$ & $\begin{array}{c}\sigma_{\min } \\
(\operatorname{arcmin})\end{array}$ & $\begin{array}{c}I \\
(\mathrm{Jy})\end{array}$ & $\begin{array}{c}\delta I \\
(\mathrm{Jy})\end{array}$ & & & & \\
\hline $0.29-0.48$ & 267.04 & -28.94 & 3.4 & 2.1 & 2.20 & 0.20 & 3.9 & 1.9 & 7.85 & 0.26 & 2.77 & 0.21 & IRAS 17449-2855 & 1 \\
\hline $1.34+0.10$ & 267.10 & -27.74 & $\ldots$ & $\ldots$ & $\ldots$ & $\ldots$ & 9.1 & 2.2 & 11.87 & 1.62 & $\ldots$ & $\ldots$ & IRAS 17419-2744 & 0 \\
\hline $0.10-0.66$ & 267.11 & -29.19 & $\ldots$ & $\ldots$ & $\ldots$ & $\ldots$ & 2.4 & 1.5 & 1.28 & 0.41 & $\ldots$ & $\ldots$ & IRAS 17419-2907 & 1 \\
\hline $1.40+0.10$ & 267.13 & -27.68 & 5.0 & 3.7 & 7.80 & 0.84 & $\ldots$ & $\ldots$ & $\ldots$ & $\ldots$ & $\ldots$ & $\ldots$ & none & 0 \\
\hline $1.18-0.07$ & 267.17 & -27.96 & 8.7 & 4.2 & 43.73 & 0.55 & $\ldots$ & $\ldots$ & $\ldots$ & $\ldots$ & $\ldots$ & $\ldots$ & IRAS 17456-2756 & 1 \\
\hline $0.54-0.62$ & 267.34 & -28.79 & 4.2 & 2.7 & 6.55 & 0.29 & 3.9 & 2.0 & 9.20 & 0.30 & 0.74 & 0.12 & IRAS 17462-2845 & 1 \\
\hline $358.28-2.03$ & 267.39 & -31.46 & $\ldots$ & $\ldots$ & $\ldots$ & $\ldots$ & 4.7 & 2.2 & 2.87 & 0.56 & $\ldots$ & $\ldots$ & IRAS 17432-3126 & 0 \\
\hline $0.37-0.79$ & 267.39 & -29.03 & 2.9 & 1.7 & 1.15 & 0.18 & $\ldots$ & $\ldots$ & $\ldots$ & $\ldots$ & $\ldots$ & $\ldots$ & IRAS 17463-2859 & 1 \\
\hline $1.63-0.04$ & 267.40 & -27.56 & 4.9 & 4.6 & 11.15 & 0.52 & 4.7 & 3.9 & 25.11 & 0.67 & 1.77 & 0.12 & IRAS $17465-2729$ & 0 \\
\hline $1.58-0.25$ & 267.57 & -27.71 & 4.1 & 2.7 & 1.83 & 1.08 & $\ldots$ & $\ldots$ & $\ldots$ & $\ldots$ & $\ldots$ & $\ldots$ & IRAS 17469-2743 & 0 \\
\hline $0.57-0.85$ & 267.58 & -28.89 & 2.3 & 1.9 & 3.14 & 0.13 & 2.0 & 1.6 & 7.65 & 0.16 & 1.94 & 0.10 & IRAS $17470-2853$ & 1 \\
\hline $1.92-0.07$ & 267.59 & -27.33 & $\ldots$ & $\ldots$ & $\ldots$ & $\ldots$ & 16.1 & 2.9 & 16.18 & 47.67 & $\ldots$ & $\ldots$ & IRAS 17443-2718 & 0 \\
\hline $1.70-0.35$ & 267.74 & -27.66 & 5.1 & 1.6 & 1.79 & 1.61 & 5.1 & 1.8 & 5.75 & 1.12 & 2.54 & 2.00 & none & 0 \\
\hline $2.50+0.03$ & 267.84 & -26.78 & $\ldots$ & $\ldots$ & $\ldots$ & $\ldots$ & 9.8 & 1.6 & 12.98 & 5.15 & $\ldots$ & $\ldots$ & IRAS 17450-2641 & 0 \\
\hline
\end{tabular}

(This table is also available in a machine-readable form in the online journal.)

(WC; Wood \& Churchwell 1989a) criterion: UCH II regions are ionized by $\mathrm{O}$ stars, which have very similar flux density distributions from object to object (Wood \& Churchwell 1989b) - the distribution of UCH II sources in the FIR color-color plane should therefore be tightly restricted, an observation which is the basis of the WC criteria of $\log \left(F_{60} / F_{12}\right) \geqslant 1.30$ and $\log \left(F_{25} / F_{12}\right) \geqslant 0.57$, where $F_{\lambda}$ is the flux density of the source in the IRAS band centered on wavelength $\lambda$, with $\lambda$ in microns. The QUaD catalog includes a field indicating whether or not each source satisfies the WC criteria; if free-free emission is important, the shape of the flux density distribution will be distorted away from that expected from pure thermal dust emission, and indicate a spectrum more consistent with a $\mathrm{UCH}$ II source. Of the 526 unique sources in the $I$ catalog, 170 satisfy the WC criterion, indicating that $32 \%$ of sources detected by QUaD have IRAS colors consistent with $\mathrm{UCH}$ II regions. This fraction may fluctuate since the WC criteria are not perfect; some real UCH II sources detected by QUaD will not be identified on this basis, while false positives may also occur. However, it does appear that a substantial fraction of QUaD sources fall into the UCH II category, and thus provide additional spectral constraints on this class of source.

\subsection{Catalog Field Description}

The fields present in the total intensity source catalog (Table 2) are designated as follows, with major and minor axes, intensities, and uncertainties tabulated for each frequency band. If a source was spatially matched between frequency bands, the $100 \mathrm{GHz}$ coordinates are quoted.

1. Source ID: source identification based on best-fit Galactic coordinates of source.

2. R.A.: source right ascension (J2000) in degrees.

3. Decl: source declination (J2000) in degrees.

4. $\sigma_{\text {maj: }}$ : major axis in $\operatorname{arcmin}(100 \mathrm{GHz})$.

5. $\sigma_{\min }:$ minor axis in arcmin $(100 \mathrm{GHz})$.

6. I: source flux density in Janskys (100 GHz).

7. $\delta I$ : source flux density uncertainty in Janskys (100 GHz).

8. $\sigma_{\text {maj }}:$ major axis in arcmin $(150 \mathrm{GHz})$.

9. $\sigma_{\min }:$ minor axis in arcmin $(150 \mathrm{GHz})$.

10. I: source flux density in Janskys (150 GHz).
11. $\delta I$ : source flux density uncertainty in Janskys (150 GHz).

12. $\alpha_{I}$ : source spectral index.

13. $\delta \alpha_{I}$ : source spectral index uncertainty.

14. Alt. name: alternative name; matched to either IRAS-PSC or PMN catalog.

15. WC: indicates whether the source satisfies the WoodChurchwell criteria for ultracompact $\mathrm{H}$ II regions.

The fields present in the polarized intensity source catalog (Table 3) are designated as follows, with total intensity and polarized intensity and angle tabulated for both frequencies.

1. Source ID: source identification based on best-fit Galactic coordinates of source.

2. R.A.: source right ascension (J2000) in degrees.

3. Decl: source decl. (J2000) in degrees.

4. I: total intensity source flux density in Janskys (100 GHz).

5. $\delta I$ : total intensity source flux density uncertainty in Janskys $(100 \mathrm{GHz})$.

6. P: polarized intensity source flux density in Janskys $(100 \mathrm{GHz})$.

7. $\delta P$ : polarized intensity source flux density uncertainty in Janskys (100 GHz).

8. $\phi$ : source polarization angle in degrees $(100 \mathrm{GHz})$.

9. $\delta \phi$ : source polarization angle uncertainty in degrees $(100 \mathrm{GHz})$.

10. I: total intensity source flux density in Janskys (150 GHz).

11. $\delta I$ : total intensity source flux density uncertainty in Janskys $(150 \mathrm{GHz})$.

12. P: polarized intensity source flux density in Janskys $(150 \mathrm{GHz})$

13. $\delta P$ : polarized intensity source flux density uncertainty in Janskys $(150 \mathrm{GHz})$.

14. $\phi$ : source polarization angle in degrees $(150 \mathrm{GHz})$.

15. $\delta \phi$ : source polarization angle uncertainty in degrees $(150 \mathrm{GHz})$.

16. $\alpha_{I}$ : total intensity source spectral index.

17. $\delta \alpha_{I}$ : total intensity source spectral index uncertainty.

18. $\alpha_{P}$ : polarized intensity source spectral index.

19. $\delta \alpha_{P}$ : polarized intensity source spectral index uncertainty.

20. Alt. name: alternative name; matched to either IRAS-PSC or PMN catalog. 
Table 3

QUaD Polarized Galactic Source Catalog

\begin{tabular}{|c|c|c|c|c|c|c|c|c|c|c|c|c|c|c|c|c|c|c|c|}
\hline \multirow[t]{2}{*}{ Source } & \multirow[b]{2}{*}{$\begin{array}{l}\text { R.A. } \\
\text { (deg) }\end{array}$} & \multirow[b]{2}{*}{$\begin{array}{l}\text { Decl. } \\
\text { (deg) }\end{array}$} & \multicolumn{6}{|c|}{$100 \mathrm{GHz}$} & \multicolumn{6}{|c|}{$150 \mathrm{GHz}$} & \multirow[b]{2}{*}{$\alpha_{I}$} & \multirow[b]{2}{*}{$\delta \alpha_{I}$} & \multirow[b]{2}{*}{$\alpha_{P}$} & \multirow[b]{2}{*}{$\delta \alpha_{P}$} & \multirow[b]{2}{*}{ Alt. Name } \\
\hline & & & $\begin{array}{c}\boldsymbol{I} \\
(\mathrm{Jy})\end{array}$ & $\begin{array}{c}\delta I \\
(\mathrm{Jy})\end{array}$ & $\begin{array}{c}\boldsymbol{P} \\
(\mathrm{Jy})\end{array}$ & $\begin{array}{l}\delta P \\
(\mathrm{Jy})\end{array}$ & $\begin{array}{c}\phi \\
(\mathrm{deg})\end{array}$ & $\begin{array}{c}\delta \phi \\
(\mathrm{deg})\end{array}$ & $\begin{array}{c}\boldsymbol{I} \\
(\mathrm{Jy})\end{array}$ & $\begin{array}{l}\delta I \\
(\mathrm{Jy})\end{array}$ & $\begin{array}{c}\boldsymbol{P} \\
(\mathrm{Jy})\end{array}$ & $\begin{array}{l}\delta P \\
(\mathrm{Jy})\end{array}$ & $\begin{array}{c}\phi \\
(\mathrm{deg})\end{array}$ & $\begin{array}{c}\delta \phi \\
(\operatorname{deg})\end{array}$ & & & & & \\
\hline $284.31-0.37$ & 156.03 & -57.81 & 143.6 & 0.3 & 2.80 & 1.09 & 13.68 & 11.15 & 138.32 & 0.36 & $\ldots$ & & .... & - & -0.08 & 0.01 & $\ldots$ & $\ldots$ & none \\
\hline $359.95-0.05$ & 266.42 & -29.01 & 175.2 & 0.4 & $\ldots$ & & $\ldots$ & $\ldots$ & 171.03 & 0.46 & 0.95 & 0.32 & 75.62 & . & -0.05 & 0.01 & $\ldots$ & $\ldots$ & none \\
\hline $0.18-0.10$ & 266.61 & -28.84 & & & 7.91 & 0.33 & -38.61 & 1.19 & & & 4.90 & 0.32 & -42.20 & 1.86 & & & -1.04 & 0.17 & IRAS $17430-2848$ \\
\hline $0.60-0.03$ & 266.80 & -28.44 & 112.7 & 0.3 & 3.21 & 7.35 & 88.40 & 65.64 & 139.83 & 0.27 & 1.33 & 0.24 & 68.57 & 5.19 & 0.47 & 0.01 & -1.92 & 5.00 & IRAS $17440-2825$ \\
\hline
\end{tabular}




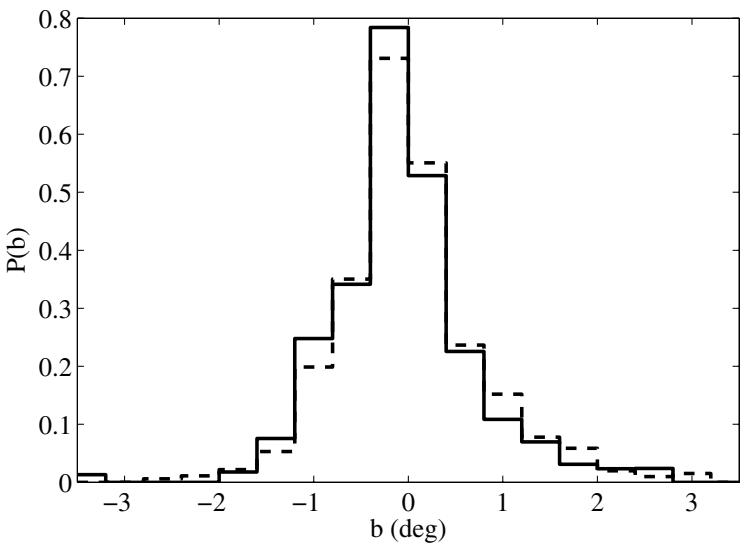

Figure 4. Probability distribution of detected sources in $I$ as a function of Galactic latitude $b$. The solid (dashed) line is for sources detected at 100 (150) $\mathrm{GHz}$. At both frequencies, the distribution peaks below $b=0$.

The typical error in each position coordinate is calculated from the distribution of position uncertainties taken over all sources; we find $\sigma_{x}={ }_{-0.1}^{+0.6} \operatorname{arcmin}$ and $\sigma_{x}={ }_{-0.1}^{+0.4} \operatorname{arcmin}$ at 100 and $150 \mathrm{GHz}$, respectively. Uncertainties in angular size from fitting each source to an elliptical Gaussian function are $\sim 0.2$ arcmin. We find typical flux uncertainties of $\sigma_{I}=0.29 \mathrm{Jy}$ and $\sigma_{I}=0.31 \mathrm{Jy}$ at 100 and $150 \mathrm{GHz}$, respectively.

\subsection{Source Distribution with Galactic Latitude}

Figure 4 shows the distribution of discrete sources as a function of Galactic latitude $b$, after correction for survey coverage (a smaller range of $b$ is sampled at lower decl.). The median of the distribution is -0.07 and -0.04 at 100 and $150 \mathrm{GHz}$, respectively. This negative offset is within one beamwidth of $b=0$ at both frequencies, but supports the results of other surveys, such as Schuller et al. (2009), who found the peak of the distribution to be -0.09 at higher $\left(19^{\prime \prime} .2\right)$ angular resolution. No obvious explanation for this offset is given, though Schuller et al. (2009) suggest the slightly positive galactic latitude of the Sun as a possible cause or alternatively the presence of molecular clouds which obscure IR sources; the latter is discussed in the context of sources near the Galactic center in Hinz et al. (2009).

\subsection{Source Counts}

Figure 5 shows the differential source counts of the catalog as a function of total intensity flux $S$. Fitting to a power-law distribution $d N / d S \propto S^{\gamma_{S}}$ in the range $10<S<300 \mathrm{Jy}$, we find $\gamma_{S, 100}=-1.8 \pm 0.4$ at $100 \mathrm{GHz}$ and $\gamma_{S, 150}=-2.2 \pm 0.4$ at $150 \mathrm{GHz}$.

If dust dominates the millimeter/submillimeter source emission, their fluxes are proportional to the masses of star-forming cores $M$ (e.g., Enoch et al. 2006), and the slope of $d N / d M$ can be used to constrain the slope of the IMF. Four caveats prevent conversion of $\mathrm{QUaD}$ source fluxes to core masses. First, the resolution required to observe individual cores (as opposed to clumps) is approximately $30^{\prime \prime}$, a factor $\sim 10$ higher than the QUaD $150 \mathrm{GHz}$ band. QUaD sources could in principle contain more than one core, biasing the measurement of core masses. Second, since the $100 \mathrm{GHz}$ band flux could contain a substantial free-free contribution, calculating masses at this frequency is not possible without further information on the relative contribution of free-free. This is less of an issue at $150 \mathrm{GHz}$, where the QUaD data should be dominated by dust. Third, the mass

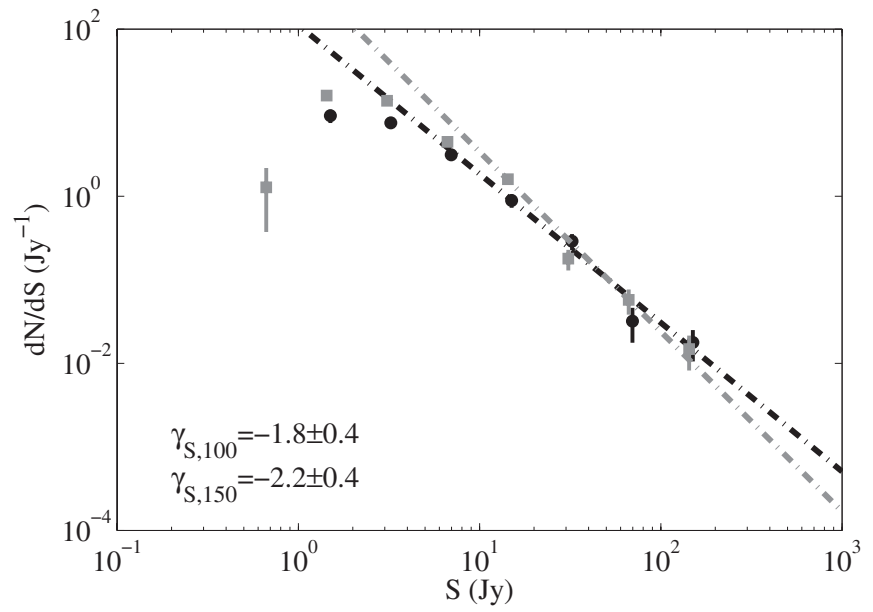

Figure 5. Differential source counts from the survey as a function of flux. Black is for $100 \mathrm{GHz}$ data; gray is for $150 \mathrm{GHz}$. The best-fit slopes are shown in the plots as dot-dashed lines with the same color coding; numerical values and uncertainties are shown in the lower left of the plot.

conversion also requires a distance estimate to the core which are not readily available for each source in the catalog. Fourth, the measurement of the slope may be subject to systematic error due to the presence of the diffuse background. Simulations in Appendix A.4.2 show that if a diffuse background is present the slope of $d N / d S$ may not be well described by a single power law. However, this effect depends on the model used for the diffuse emission in the simulations, namely, the amplitude of the background relative to the sources, and the power in diffuse substructure. Caution is thus advised when interpreting the slope results quoted above, though Figure 5 indicates that power-law behavior is observed above $10 \mathrm{Jy}$, and thus the contribution of diffuse emission in this flux regime is not important.

Due to these caveats, we caution against overinterpretation of the measured slope of $d N / d S$ and refrain from assigning a mass to each source and from converting the slope of $d N / d S$ to the slope of the IMF.

\subsection{Spectral Index Distribution}

The spectral index distribution in total intensity, $\operatorname{Pr}\left(\alpha_{I}\right)$, is computed following Muchovej et al. (2010). For each source $j$, the spectral index probability distribution $\operatorname{Pr}_{j}\left(\alpha_{I}\right)$ is calculated by generating flux distributions at each frequency from the central value and noise distributions, and then combining the flux distributions. The spectral index distribution for the sample is then the normalized sum of the $\operatorname{Pr}_{j}\left(\alpha_{I}\right)$, i.e.,

$$
\operatorname{Pr}\left(\alpha_{I}\right)=\frac{\sum_{j} \operatorname{Pr}_{j}\left(\alpha_{I}\right)}{\int \sum_{j} \operatorname{Pr}_{j}\left(\alpha_{I}\right)}
$$

Figure 6 shows $\operatorname{Pr}\left(\alpha_{I}\right)$ for sources matched between the QUaD bands.

The spectral index of the sources are somewhat flatter than those found at higher frequencies (e.g., Désert et al. 2008), peaking at $\alpha_{I} \sim 0.25$; this could be due to the contribution from other emission components at $100 \mathrm{GHz}$, raising the flux at this frequency above that expected from dust alone and therefore flattening the spectral index. Simulations indicate that the spectral index distribution can be slightly skewed toward larger $\alpha_{I}$ by background contamination and source confusion (see Appendix A.4.3); the center of the distribution shifts by 


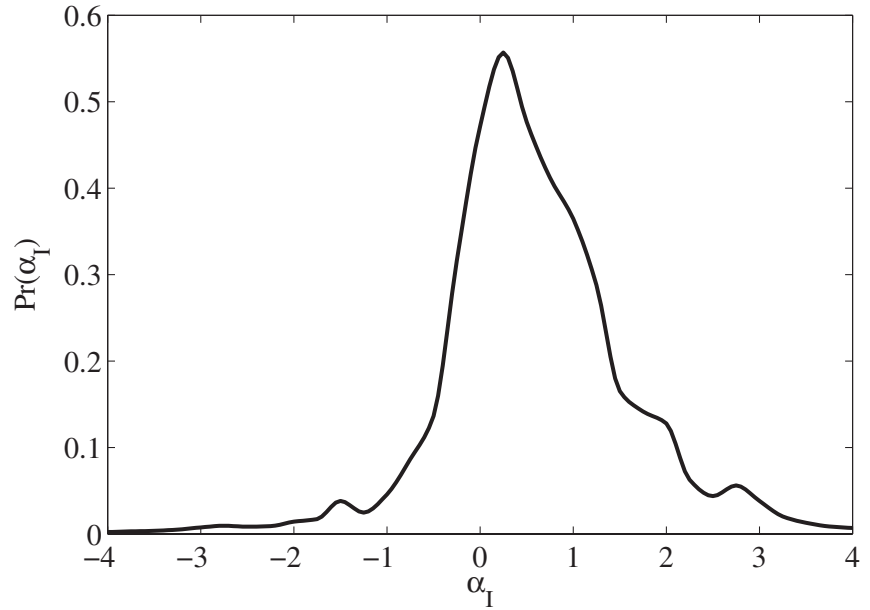

Figure 6. Source total intensity spectral index distribution $\operatorname{Pr}\left(\alpha_{I}\right)$.

$\sim 0.2$ in $\alpha_{I}$. Finally, there is evidence of unaccounted emission processes at $100 \mathrm{GHz}$ in the QUaD data (see Map Paper), which would also shift the spectral index distribution to lower values; detected sources are generally not faint enough that flux boosting is important.

Our analysis does not account for flux boosting due to noise on account of the larger (systematic) effect of contamination due to the diffuse background and map filtering effects. Rigorous Bayesian methods to determine the spectral index distribution of sources exist in the literature (e.g., Crawford et al. 2010; Vieira et al. 2010), but do not account for the effect of an unknown background, which is the largest contaminant to source fluxes in the galaxy as demonstrated in Appendix A.4.1. We therefore do not pursue such an approach; the increased frequency coverage of current-generation satellite experiments such as Planck and Herschel may allow an improved treatment of the diffuse background, enhancing the extraction of discrete Galactic sources and their spectral indices.

\subsection{Source Clustering}

Figure 1 demonstrates that source locations in the QUaD survey are highly correlated. To quantify source clustering, we construct the two-point angular correlation function $w(\theta)$, defined as the excess probability of finding a source within angle $\theta \pm \Delta \theta$ of another source, $H_{d}(\theta)$, compared to the same probability in a distribution of sources with random spatial positions, $H_{r}(\theta)$ :

$$
w(\theta)=\frac{H_{d}(\theta)}{H_{r}(\theta)}-1
$$

Enoch et al. (2006) model $w$ as a power law in units of projected physical separation $r, w(r) \propto r^{\gamma_{r}}$, and use the slope as a method of comparing the spatial properties of cores in different molecular clouds; the authors suggest that different slopes may provide insight into the processes dominating core fragmentation. In the QUaD survey, we compute the correlation function in angular units $w(\theta) \propto \theta^{\gamma_{\theta}}$, by constructing $H_{d}(\theta)$ from the data, and $H_{r}(\theta)$ from one realization of Sim1 (a simulation with sources distributed randomly over the QUaD survey). Differing survey areas at each frequency and variations in survey sensitivity are then accounted for.

Any survey over a large range of Galactic latitude faces the problem that the distribution of sources is anisotropic,

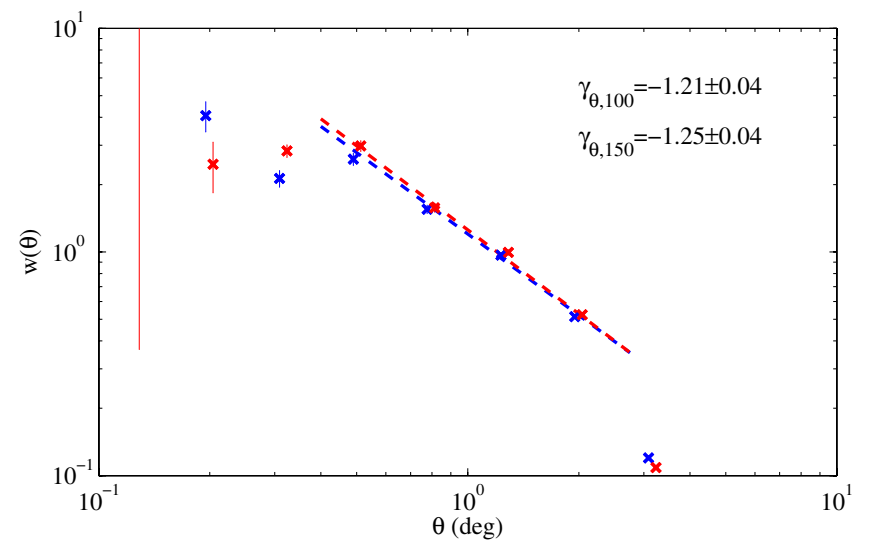

Figure 7. Angular correlation function $w(\theta)$ for sources in QUaD survey; blue is for $100 \mathrm{GHz}$ data, red is $150 \mathrm{GHz}$. Only fourth quadrant data are used because the gap in survey coverage between the third and fourth quadrants introduces a discontinuous range of source Galactic longitudes. Since the number of sources in the third quadrant is only a small fraction of the total survey, the effect on the analysis is small.

(A color version of this figure is available in the online journal.)

with $w(\theta)$ poorly defined at large Galactic latitudes due to the shape of the galaxy projected on the sky. In addition, at much smaller separations $w(\theta)$ is not well reconstructed due to the large probability that a neighboring source has a low flux, assuming a power-law source count $d N / d S \propto S^{\gamma_{S}}$, with $\gamma_{S}<0$. Therefore, though a bright source will be detected in the survey, its fainter neighbor is likely to lie below the noise or confusion limit, preventing accurate reconstruction of $w(\theta)$ at small $\theta$. Simulations indicate that $w(\theta)$ is well recovered in the range $0.4<\theta<2^{\circ}$ (see Appendix A.4.4) and therefore these limits are used to fit a power law to the correlation function of the data. Only sources from the fourth quadrant are used, because the gap in survey coverage between the third and fourth quadrants introduces artifacts into $w$; since sources in the third quadrant account for $<20 \%$ of all sources at each frequency, the calculated slope is not affected by removal of these sources.

Figure 7 shows the results. We find a power-law slope of $\gamma_{\theta, 100}=-1.21 \pm 0.04$ and $\gamma_{\theta, 150}=-1.25 \pm 0.04$, consistent with the value found by Enoch et al. (2006) for the Bolocam observations of the Perseus molecular cloud, $w(r) \propto r^{-1.25}$ (since a single distance is assumed to Perseus, there is a one-one mapping between $r$ and $\theta$, and thus the spatial correlation function of Enoch et al. (2006) can be easily converted to an angular correlation function comparable to the QUaD result). This similarity implies that the same correlation function slope applies to sources on large and small angular scales (QUaD and Bolocam surveys, respectively). However, it should be noted that due to differing resolutions, Bolocam and QUaD measure the angular correlation function of different types of sources-cores in the case of Bolocam and clumps in the case of QUaD. Therefore, while the correlation function slopes are consistent, it is not clear that the correlation functions measured by each experiment are directly related, and thus caution is advised when comparing these results.

\subsection{Polarized Sources}

Polarized sources are of particular interest due to their scarcity and the fact that they offer a means for probing smallscale magnetic field structure in the Galaxy. Maps of detected polarized sources are examined visually in order to reject beamscale optical effects (such as beam offsets and/or differing 

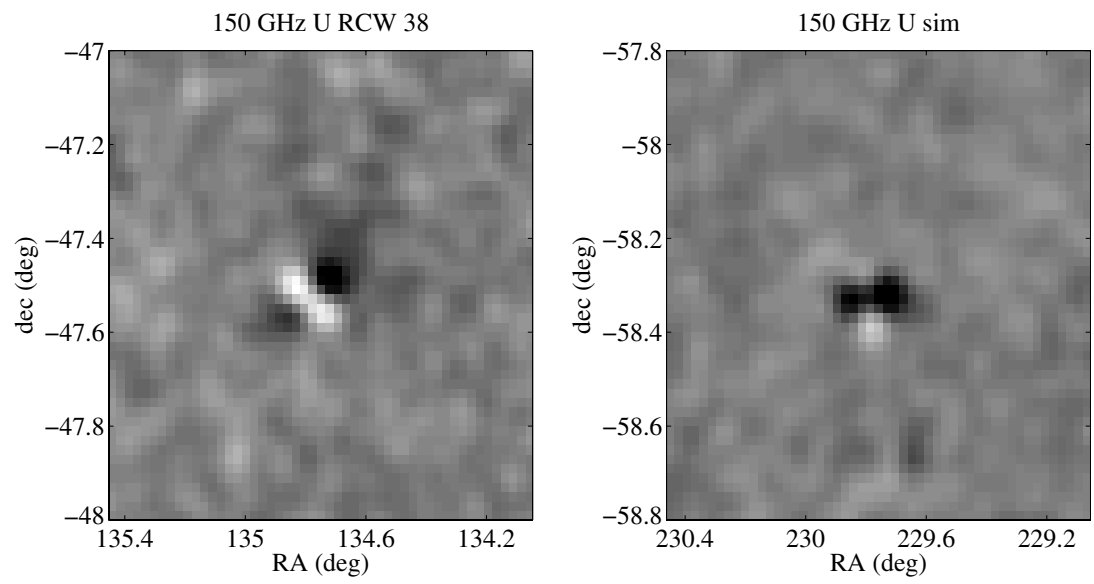

Figure 8. Illustration of the optical effect for low $(<1 \%)$ fractional polarization sources. Left is a $150 \mathrm{GHz} U$ map centered on RCW 38 ; right is a $150 \mathrm{GHz} U$ map of a simulated source showing a similar optical effect. The color scale on each plot is the same.
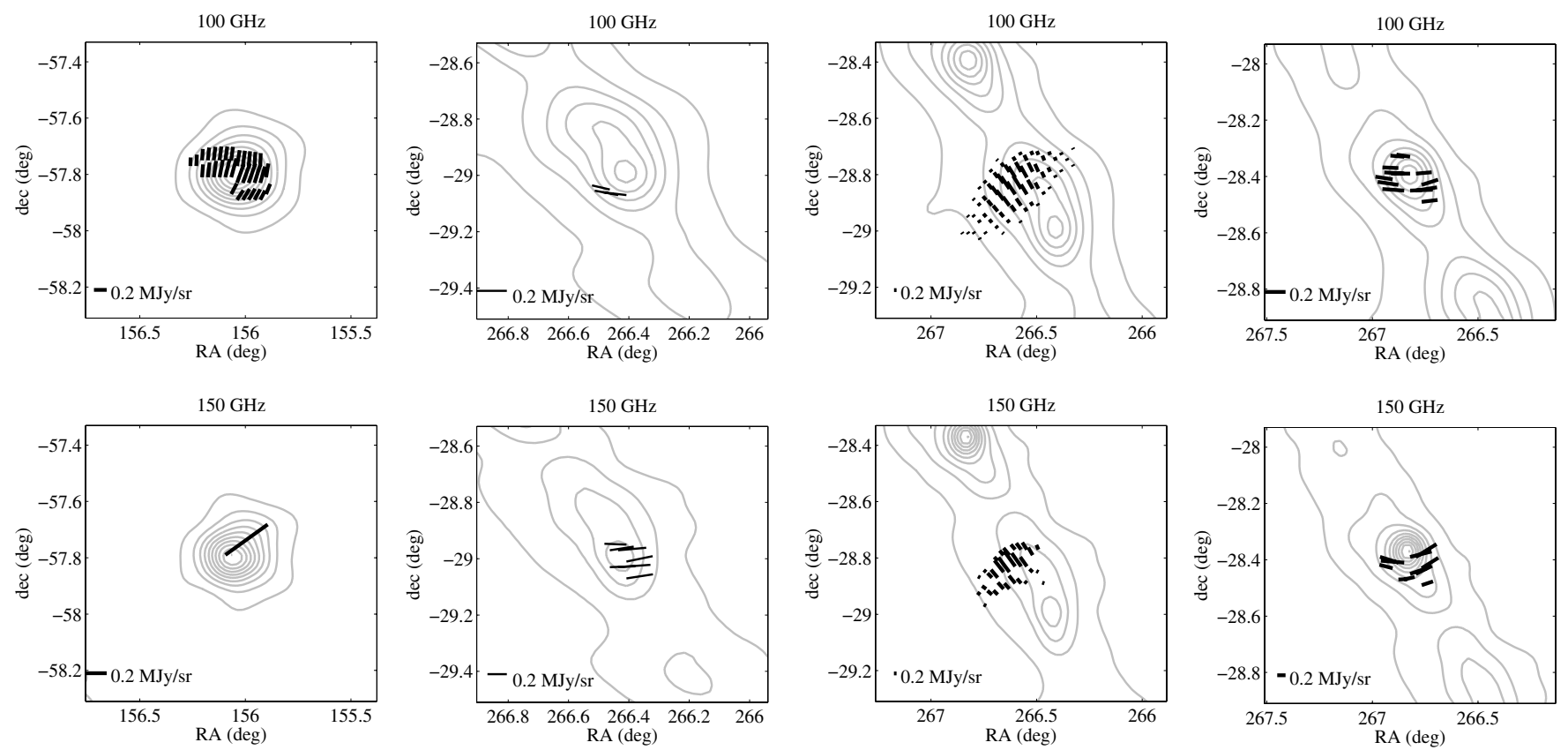

Figure 9. Total intensity images with polarization vectors overlaid on each source detected in polarized intensity-source fluxes, spectral indices, and alternative identifications are presented in Table 3. Only polarization vectors with a signal-to-noise ratio greater than 5 are plotted, and the polarization vectors are down sampled for clarity, such that only every third vector is plotted. Top row is $100 \mathrm{GHz}$, bottom row is $150 \mathrm{GHz}$, only polarization vectors with signal-to-noise $>5$ are plotted. From left to right: $284.31-0.37$ (RCW 49), 359.95-0.05 (Sagittarius A*), 0.18-0.10 (Galactic Center Arc), and 0.60-0.03 (Sagittarius B2). For 284.31-0.37, the contours run from 1.6 to $16.1 \mathrm{MJy} \mathrm{sr}^{-1}$ in steps of $1.6 \mathrm{MJy} \mathrm{sr}^{-1}$ at $100 \mathrm{GHz}$, and from 2.3 to $23.3 \mathrm{MJy} \mathrm{sr}^{-1}$ in steps of $2.3 \mathrm{MJy} \mathrm{sr}^{-1}$ at $150 \mathrm{GHz}$. For the remaining sources, the contours run from 2 to $20 \mathrm{MJy} \mathrm{sr}^{-1}$ in steps of $2 \mathrm{MJy} \mathrm{sr}^{-1}$ at $100 \mathrm{GHz}$, and from 5 to $50 \mathrm{MJy} \mathrm{sr}^{-1}$ in steps of $5 \mathrm{MJy} \mathrm{sr}{ }^{-1}$ at $150 \mathrm{GHz}$.

beam ellipticities between two PSBs within a feed). These can cause spurious source detection at fractional polarization $(\sim 1 \%$ or less $)$ - see the Instrument Paper for further details. Figure 8 shows the $150 \mathrm{GHz} U$ map of RCW 38 and a simulated point source of low fractional polarization. The "quadrupole" polarization pattern is observed in the two cases, implying that the apparent polarization of RCW 38 is an instrumental effect rather than real polarized signal. Polarized sources exhibiting such a pattern are visually rejected from the catalog.

Properties of the remaining polarized sources in the QUaD survey are presented in Table 3, with images of each in total and polarized intensity in Figure 9. Below we discuss each source in more detail.

\subsection{1-0.37: $R C W 49$}

RCW 49 is a bright $\mathrm{H}$ II region covering $90^{\prime} \times 70^{\prime}$, which is being ionized by the rich, compact star cluster Westerlund 2 (e.g., Furukawa et al. 2009). Numerous total intensity observations exist from the radio to X-ray wavebands; however, existing studies of this source in polarization near the $\mathrm{QUaD}$ bands have been restricted to Dickinson et al. (2007), who observed several southern $\mathrm{H}$ II regions with the CBI telescope at $31 \mathrm{GHz}$. Their measurements of RCW 49, at 6.78 arcmin angular resolution, provide an upper limit on the $31 \mathrm{GHz}$ polarization fraction of $0.24 \%$, limited by instrumental leakage from Stokes $I$ to $Q$ and $U$. Below $31 \mathrm{GHz}$, the RCW 49 emission is dominated by free-free, as indicated by the total intensity spectral index $\alpha_{I, \mathrm{RCW} 49}=-0.220 \pm 0.074$ between 2.7 and $15 \mathrm{GHz}$ (Dickinson et al. 2007). The QUaD total intensity counterpart to RCW 49, 284.31-0.37, also indicates a flat spectral index between 100 and $150 \mathrm{GHz}$ of $\alpha_{I, \mathrm{RCW} 49, \mathrm{QUaD}}=-0.08 \pm-0.01$. Polarized emission is detected in the QUaD data at $100 \mathrm{GHz}$ with a polarization fraction of $0.019 \pm 0.0076$ and the polarization vectors aligned predominantly north-south (see Figure 9). 
The absence of detected polarization at $150 \mathrm{GHz}$ indicates that the emission may not be thermal in nature; this idea is supported by the total intensity spectral index, though this measurement is likely biased flat by the presence of free-free emission. While free-free is not intrinsically polarized, it may cause polarization by Thomson scattering at the edges of the $\mathrm{H}$ II region, resulting in tangentially polarized radiation at the cloud edges. Since the QUaD $100 \mathrm{GHz}$ polarization vectors are largely aligned over the source area, free-free polarization at the cloud edges can be ruled out. Synchrotron radiation is a further possibility, but unlikely given the physical nature of the source. It is therefore possible that instrumental effects other than those illustrated in Figure 8 are present.

\subsection{5-0.05: Sagittarius $A^{*}$}

High frequency polarized observations of Sagittarius A* (Sgr $A^{*}$, detected in the $\mathrm{QUaD}$ survey as 359.95-0.05) are important due to the constraints they provide on relativistic jets and accretion processes in black holes (e.g., Agol 2000; Quataert \& Gruzinov 2000; Melia et al. 2001). The rotation measure (RM), which is proportional to the electron density and magnetic field component integrated along the line of sight, can be determined from multi-frequency observations of the linear polarization fraction-measurements of the RM constrain the mass accretion rate of the black hole and thus rule out certain classes of accretion model. Observations of linear polarization in Sgr A*, first detected with SCUBA by Aitken et al. (2000), have been studied with interferometric imaging at frequencies above $\sim 100 \mathrm{GHz}$, typically with resolutions $<10^{\prime \prime}$ or better (Macquart et al. 2006; Marrone et al. 2007). At $3.5 \mathrm{~mm}$, Macquart et al. (2006) find a fractional linear polarization of $2.1 \% \pm 0.1 \%$, while larger values are found at higher frequencies $(\sim 5 \%$ and $\sim 9 \%$ at 230 and $340 \mathrm{GHz}$, respectively; Marrone et al. 2007). Aitken et al. (2000) measured an observed polarization fraction of $2.9 \% \pm 0.3 \%$ and polarization angle of $84^{\circ} \pm 3^{\circ}$ at $2 \mathrm{~mm}$ with 33 ".5 resolution.

In the QUaD data, Sgr $\mathrm{A}^{*}$ is detected in polarization at $150 \mathrm{GHz}$ only (see Figure 9 and Table 3), but the polarized flux is unconstrained; it appears that the polarization fraction is $<1 \%$ at $150 \mathrm{GHz}$, considerably less than that found by interferometric instruments at comparable frequencies. This result is not surprising, given the factor of $\sim 20$ lower resolution compared to BIMA at $3.5 \mathrm{~mm}$ (Macquart et al. 2006) and a factor of $\sim 6$ lower than SCUBA at $2 \mathrm{~mm}$ (Aitken et al. 2000). At 3.5 angular resolution at $150 \mathrm{GHz}$, the polarized emission from Sgr A* is smeared out and the polarization fraction is therefore reduced due to the contribution from the diffuse background or unpolarized sources within a QUaD $150 \mathrm{GHz}$ beam of Sgr A*. Though the QUaD polarization angle from the elliptical Gaussian fit is unconstrained (see Table 3), the statistically significant $150 \mathrm{GHz}$ polarization vectors shown in Figure 9 indicate a similar value to the Aitken et al. (2000) polarization angle result. While the QUaD observations span four months and could constrain the variability of the polarized emission (a useful diagnostic of processes intrinsic to the source), at such low resolution and signal-to-noise it is not possible to measure this effect. The apparent low polarization fraction of this source may result in false detection due to an optical effect in the telescope, similarly to $284.31-0.37$, and further interpretation of the polarization of this source should therefore be treated with caution.

\subsection{8-0.10: The Galactic Center Arc}

$0.18-0.10$ is clearly associated with G0.2-0.0, also known as the Galactic Center Arc. This source has an extent of $\sim 25$ arcmin along its long axis perpendicular to the Galactic plane, is approximately symmetric with respect to the Galactic equator, and is among the brighter sources close to the Galactic center (e.g., Altenhoff et al. 1979; Lis \& Carlstrom 1994). High resolution observations (e.g., Yusef-Zadeh et al. 2004) indicate that this filamentary structure contains the largest concentration of non-thermal radio filaments in the Galaxy. Though this object appears extended and unassociated with any discrete source in the QUaD $I$ data (see Figure 9 and Table 3), the radio filaments cross three bright $\mathrm{H}$ II regions unresolved by QUaD (e.g., Yusef-Zadeh et al. 1986; Reich et al. 2000): G0.16-0.15, G0.18-0.04, and G0.1+0.08. Using the Green Bank Telescope, Law et al. (2008) determined that this radio arc has a nonthermal spectrum of $-0.54 \pm 0.09$ between 4.85 and $8.5 \mathrm{GHz}$ (resolution 2.5 and 1 arcmin, respectively), in support of the idea that the emission is not from cold dust, but rather from either monoenergetic electrons or an electron distribution with a low energy cutoff (Reich et al. 2000). Observations at 4.8 GHz using the Very Large Array (Yusef-Zadeh et al. 1986) demonstrate that while thermal emission dominates G0.18-0.04 and G0.1+0.08, the low-frequency non-thermal polarized emission in the arc is primarily due to the $\mathrm{H}$ II region $\mathrm{G} 0.16-0.15$; the high $(\sim 30 \%)$ fractional polarization observed provides further evidence that the polarized emission is due to synchrotron emission.

We find a peak polarization fraction of $\sim 10 \%$ at both 100 and $150 \mathrm{GHz}$ and a polarized spectral index of $-1.04 \pm 0.17$, indicative of a source dominated by synchrotron emission. Since we do not detect a discrete total intensity source associated with the polarized arc, we estimate the polarization fraction from the raw $I$ images and the polarized flux measured by the source extraction algorithm. Due to its spatial extent above the beam scale in the QUaD survey, it is doubtful that the radio arc's polarized emission at these frequencies can be solely attributed to G0.16-0.15, which would likely be unresolved. The high level of uniformity of the polarization seen in Figure 9 indicates a highly ordered magnetic field which must exist over the full extent of the arc, rather than localized to a single $\mathrm{H}$ II source. The polarized vectors are largely aligned parallel to the plane of the Galaxy, almost perpendicular to the polarization from diffuse emission (see Map Paper), indicating a strong local deviation from the galactic magnetic field.

\subsection{0-0.03: Sagittarius B2}

The polarization of Sagittarius B2 (Sgr B2) has been well studied in the radio, submillimeter, and far-infrared (e.g., Greaves et al. 1995; Dowell 1997; Novak et al. 1997; Dowell et al. 1998; Jones et al. 2011); the QUaD data fill in the millimeter portion of the spectrum of this giant molecular cloud. Observations of linearly polarized emission from magnetically aligned dust grains in such clouds can be used to determine the orientation of the local magnetic field. In the submillimeter, where the dust is optically thin, the polarization is due to emission of the dust grains preferentially along their long axis; Greaves et al. (1995) observed Sg B2 at $800 \mu \mathrm{m}$ and 30" resolution, finding polarization fractions in the range $0.8 \%-2.6 \%$ with the polarization orientation approximately north-south. Polarization observations at FIR wavelengths include both emission and absorption effects. The former is due to dust grain emission similarly probed by sub-mm observations and results in 

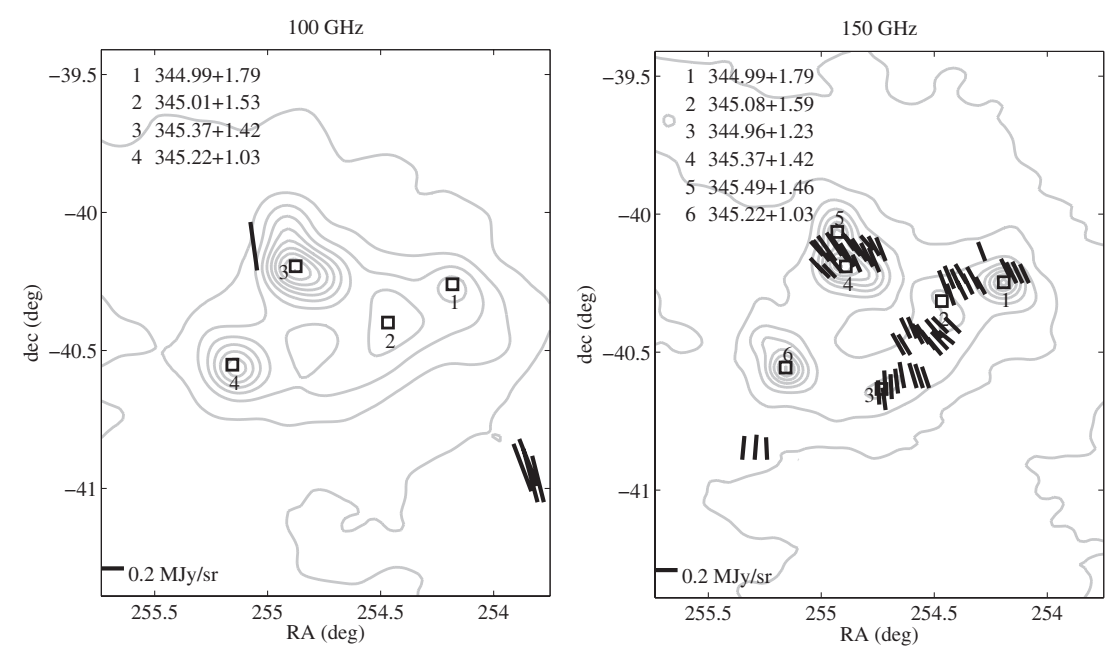

Figure 10. Large-scale polarized cloud at $100 \mathrm{GHz}$ (left) and $150 \mathrm{GHz}$ (right) undetected by source extraction algorithm-only polarization vectors with signal-to-noise $>4$ are plotted (black lines). At $100 \mathrm{GHz}$, the contour scale (gray lines) runs from 0.32 to $3.2 \mathrm{MJy} \mathrm{sr}^{-1}$ in steps of $0.32 \mathrm{MJy} \mathrm{sr}^{-1}$, and from 0.77 to $7.7 \mathrm{MJy} \mathrm{sr}{ }^{-1}$ in steps of $0.77 \mathrm{MJy} \mathrm{sr}^{-1}$ at $150 \mathrm{GHz}$. Black squares indicate the locations of discrete sources detected in the $I$ maps, with the names of the sources as indicated at upper left of each plot.

polarization vectors aligned perpendicular to the local magnetic field direction, while the latter is caused by absorption from cold, magnetically aligned dust grains in regions of high optical depth, with corresponding polarization vectors aligned parallel to the magnetic field. Novak et al. (1997) demonstrate that at $115 \mu \mathrm{m}$ with a $35^{\prime \prime}$ beam, Sgr B2 is resolved into "core" and "envelope" regions, with polarization in the core and envelope dominated by absorption and emission, respectively. In their study, the envelope fractional polarization ranges from $2 \%$ to $4 \%$. At $350 \mu \mathrm{m}$, the effects of absorption are diminished due to the increasing contribution of dust emission and the decreasing optical depth; Dowell et al. (1998) show this observationally, with the core polarization ( $\sim 1 \%$ fractional polarization) smaller than that in the envelope $(\sim 2.8 \%)$ at $20^{\prime \prime}$ resolution.

Based on the above considerations, in the QUaD bands the polarization of Sgr B2 should be dominated by dust grain emission processes. The best measurement of polarization fraction is at $150 \mathrm{GHz}$, where we find a value of $1 \% \pm 0.2 \%$. It is not surprising that the $150 \mathrm{GHz}$ polarization fraction is smaller than at $350 \mu \mathrm{m}$. Since the QUaD beam at this frequency is a factor $3.5 \times 60 / 20 \simeq 11$ larger, the core and envelope are not resolved into separate components, and thus the higher envelope polarization fraction is biased low by the less strongly polarized core region. The east-west orientation of the $150 \mathrm{GHz}$ QUaD polarization vectors (see Figure 9), similar to those at $350 \mu \mathrm{m}$ (Dowell et al. 1998), further indicates that we are observing polarized dust emission from the envelope.

\subsubsection{An Extended Polarized Source: Further Filtering Considerations}

For the sake of simplicity, the same aggressive background filtering (small $\sigma_{\text {bck }}$ relative to $\sigma_{\text {beam }}$ ) is used in both total and polarized intensity. This choice was motivated by the bright diffuse emission in total intensity: a larger $\sigma_{\text {bck }}$ would result in a smaller systematic loss of signal from point sources but an increased contribution from the background-see Appendix A.5. However, this choice may be relaxed in polarized intensity because the polarized diffuse emission is relatively faint (polarization fraction $<2 \%$, see Map Paper). The present catalog is therefore not optimized for the detection of polarized sources, and more sources could in principle be extracted from the maps.
As an illustration, Figure 10 shows total and polarized intensity maps for a visually identified cloud with appreciable polarization at $150 \mathrm{GHz}$ (up to $10 \%$ fractional polarization). Since this source has a relatively large extent in comparison to the beam scale, it is undetected by the source extraction algorithm because most of the polarized flux is filtered out in the background removal step.

This object is host to several discrete sources, namely, $344.99+1.79, \quad 345.01+1.53, \quad 345.37+1.42$ (resolved into $345.38+1.41$ and $345.49+1.46$ at $150 \mathrm{GHz}), 345.22+1.03$, and $344.96+1.23$ (detected at $150 \mathrm{GHz}$ only). Sources $345.01+1.53$ and $345.08+1.59$, detected at 100 and $150 \mathrm{GHz}$ respectively, are clearly associated with the same source, but have observed centroids separated by more than the 0.02 required to meet the spatial matching criterion. The polarized emission in this region is only partially correlated with the positions of the discrete $I$ sources (see Figure 10), and the polarization vectors are largely oriented perpendicular to those from the bulk polarized galactic emission. This indicates that the diffuse dust is subject to a local magnetic field strong enough to overcome that of the galaxy as a whole, despite its small galactic latitude of $\sim 1$.4. Clearly this source is worthy of detailed follow-up study.

\section{DISCUSSION AND CONCLUSIONS}

We present a catalog of discrete sources extracted from the QUaD galactic plane survey, which spans approximately $245^{\circ}-295^{\circ}$ and $315^{\circ}-5^{\circ}$ in galactic longitude $l$ and $-4^{\circ}$ to $+4^{\circ}$ in galactic latitude $b$ - a total of $\sim 800 \mathrm{deg}^{2}$ coverage in Stokes $I$, $Q$, and $U$ at 100 and $150 \mathrm{GHz}$, with resolution 5 and 3.5 arcmin, respectively. Simulations of a toy model galaxy including spatially clustered point sources and diffuse emission indicate a $90 \%$ completeness flux of $5.2(2.8) \mathrm{Jy}$ at $100(150) \mathrm{GHz}$ in $I$, and $1.2(0.9) \mathrm{Jy}$ in polarization at $100(150) \mathrm{GHz}$. At a signal-tonoise threshold of 5 (3) in total (polarized) intensity, the catalog is $98 \%$ pure in $I$ at both frequencies and $97 \%(92 \%)$ pure in polarization at $100(150) \mathrm{GHz}$. Simulations without a diffuse background are used in the total intensity computation because substructure in diffuse emission, detected as discrete sources, biases the purity low to $\sim 45 \%$. This low value is a function of the diffuse emission model parameters, which do not perfectly reflect the amount of substructure in the galaxy. Thus, the total 
intensity catalog purity is likely in the range $45 \%-98 \%$. The percentage of IRAS-PSC counterparts to QUaD sources $(80 \%$ and $55 \%$ at 100 and $150 \mathrm{GHz}$, respectively) indicates that some low flux-density $100 \mathrm{GHz}$ QUaD sources may be due to resolved substructure in the diffuse background. The lower fraction of $150 \mathrm{GHz}$ counterparts is more likely due to resolution of a single IRAS source into multiple components by the higher-resolution $150 \mathrm{GHz}$ QUaD data. The polarized diffuse background, with fractional polarization $\sim 2 \%$ (see Map Paper), is faint enough that it does not bias the purity of the catalog at the signal-to-noise threshold of the survey. Instrumental effects prevent detection of polarized sources with polarization fraction $\sim 1 \%$ or less.

In total intensity the catalog contains 526 unique sources, of which 239 are spatially matched between frequency bands, with 53 (234) detected at $100(150) \mathrm{GHz}$ alone.

The $I$ flux distributions are well approximated by a power law over more than two orders of magnitude above $\sim 10 \mathrm{Jy}$ at both frequencies. We find power-law slopes of $\gamma_{S, 100}=-1.8 \pm 0.4$ at $100 \mathrm{GHz}$ and $\gamma_{S, 150}=-2.2 \pm 0.4$ at $150 \mathrm{GHz}$; the latter is consistent with Rosolowsky et al. (2010), who find $-2.4 \pm 0.1$ at $268 \mathrm{GHz}$ with Bolocam at higher resolution. The flatter slope at $100 \mathrm{GHz}$ may be the result of resolution effects due to the larger beam at this frequency. Simulations indicate that if the diffuse background contributes spurious sources, as expected the recovered source flux distribution does not accurately follow the underlying distribution; however, as discussed above, the high percentage of QUaD sources spatially matched to IRAS indicates that this effect is insignificant.

The spectral index probability distribution of sources in total intensity is found to peak at $\alpha \sim 0.25$, flatter than expected for sources whose emission is dominated by thermal dust. Simulations indicate that the diffuse background does not strongly influence source spectral indices; the flatness is therefore likely due to free-free emission, which becomes significant at $\sim 100 \mathrm{GHz}$ and below. At this frequency, free-free emission results in higher fluxes than expected from dust alone, shifting the spectral index distribution to lower values.

We explore the clustering of Galactic sources by fitting the two-point correlation function to a power law using the $I$ source locations. Simulations indicate that the underlying correlation function slope can be accurately reconstructed in the range $0.4<\theta<2^{\circ}$, with $\theta$ being the angular separation between a pair of sources. The correlation function breaks down at larger angular scales because so few $(<1 \%)$ of sources are located beyond $|b|=3^{\circ}$. At angular separations smaller than $0.4, w(\theta)$ is not well reconstructed because for a power law $d N / d S \propto S_{S}^{\gamma}$ with $\gamma_{S}<0$, the survey does not detect most neighbors of a source bright enough to be included-one must extend the search to large angular separations before enough neighbors are detected for accurate reconstruction. Fitting to the QUaD $I$ catalog data in the range $0.4<\theta<2^{\circ}$, we find power-law slopes of $\gamma_{\theta, 100}=-1.21 \pm 0.04$ and $\gamma_{\theta, 150}=-1.25 \pm 0.04$ at 100 and $150 \mathrm{GHz}$, respectively. These are consistent with the value found by Enoch et al. (2006) Bolocam observations of the Perseus molecular cloud, $w(\theta) \propto \theta^{-1.25}$, though the results are not directly comparable on account of the different sources probed by QUaD (clumps) and Bolocam (cores) due to their differing angular resolution. A comparison of the source correlation function in units of physical separation $r$ is not presently possible due to the wide range of unknown clump distances in the QUaD data.

Eighty percent $(55 \%)$ of the sources detected at 100 (150) $\mathrm{GHz}$ have IRAS-PSC counterparts. These fractions in- dicate that most of the clumps detected in the survey are past the prestellar phase and have envelopes heated by protostars. This observation might be expected, given that the QUaD frequency bands lie far from the spectral peak. Only these sources are bright enough in the Rayleigh-Jeans portion of the spectrum to be detected in the QUaD survey, unlike prestellar or starless sources.

Since the QUaD survey is sensitive to free-free emission as well as dust, particularly in the $100 \mathrm{GHz}$ band, sources might also be detected if their gas is sufficiently ionized to produce free-free emission but their envelopes are yet to thermalize. However, since $80 \%$ of $100 \mathrm{GHz}$ QUaD sources are matched to IRAS-PSC, most of the detected sources at this frequency appear to have a thermal component. At $150 \mathrm{GHz}$, the larger unmatched fraction is likely due to single IRAS sources being resolved into two sub-clumps by the higher QUaD resolution at this frequency-only one of these sub-clumps can be spatially matched to the IRAS source. Of the sources with an IRAS counterpart, 170 satisfy the WC criteria for UCH II regions (Wood \& Churchwell 1989a), providing new spectral constraints on this class of object. Four compact polarized sources were detected by the automated source-finding algorithm: $284.31-0.37$ (RCW 49), 359.95-0.05 (Sagittarius A*), 0.18-0.10 (IRAS 17430-2848 or Galactic Center Arc), and 0.60-0.03 (IRAS 17440-2825 or Sagittarius B2). One additional extended source was located "by eye" from the raw $Q$ and $U$ maps; this object appears to host several discrete total intensity sources, including $344.99+1.79,345.01+1.53,345.37+1.42$, and $345.22+1.03$. The brightest polarized source is $0.18-0.10$, which does not have an obvious discrete counterpart in total intensity, but has a polarization fraction of $\sim 10 \%$ if the diffuse background is used as a measure of $I$. It has a polarized flux of $7.91 \pm 0.33$ $(4.90 \pm 0.32) \mathrm{Jy}$ at $100(150) \mathrm{GHz}$ and a polarized spectral index of $\alpha_{P}=-1.04 \pm 0.17$, indicating a synchrotron emission source. Its detection against a polarized background implies that there is a strong local deviation from the galactic magnetic field.

Less than $1 \%$ of the sources detected in $I$ have a polarized counterpart. If discrete sources do not harbor strong local magnetic fields or shielding, dust grains in their envelopes will align with the large-scale Galactic field. The only way to separate diffuse from discrete polarized emission would then be via morphology (similar to $I$ ) or spectrally, since the orientation of the polarization would be similar for diffuse and discrete sources. Alternatively, the discrete total intensity sources may have fractional polarization $<1 \%$, as might be expected from a star-forming clump, in which case instrumental effects prevented detection of their polarized emission here. Discrete sources may therefore not be a significant contributor to the low-latitude galactic polarized emission. More sensitive observations (such as from the Planck satellite) will be needed to better study the polarization of these sources and the role of magnetic fields in star-forming regions.

The QUaD catalog may prove useful for a variety of additional purposes. Total intensity source fluxes could better measure the continuum spectra of clumps in conjunction with independent data sets, improving the separation of different emission components and tightening constraints on dust emissivity and gas temperatures. The maps provide upper limits to source polarization, allowing a statistical study of polarized contribution to anomalous emission similar to Dickinson et al. (2007). Finally, the catalog provides a cross-check of astrometry and absolute calibration for instruments with access to the southern hemisphere. 
Table 4

Simulation Summary

\begin{tabular}{|c|c|c|c|c|c|c|c|c|c|c|}
\hline \multirow[t]{2}{*}{ Simulation } & \multirow{2}{*}{$\begin{array}{c}\text { Source Spatial } \\
\text { Distribution }\end{array}$} & \multirow{2}{*}{$\begin{array}{c}\text { Diffuse } \\
\text { Emission }\end{array}$} & \multicolumn{4}{|c|}{$90 \%$ Completeness } & \multicolumn{4}{|c|}{ Purity $^{a}$} \\
\hline & & & $I_{100}(\mathrm{Jy})$ & $I_{150}(\mathrm{Jy})$ & $P_{100}(\mathrm{Jy})$ & $P_{150}(\mathrm{Jy})$ & $p_{I, 100}$ & $p_{I, 150}$ & $p_{P, 100}$ & $p_{P, 150}$ \\
\hline Sim1 & random & no & 1.2 & 1.3 & 0.9 & 0.9 & 0.88 & 0.89 & 0.90 & 0.91 \\
\hline $\operatorname{Sim} 2$ & random & yes & 1.3 & 1.4 & 0.9 & 0.9 & 0.43 & 0.40 & 0.93 & 0.91 \\
\hline $\operatorname{Sim} 3$ & correlated & no & 4.5 & 2.3 & 1.1 & 0.9 & 0.98 & 0.98 & 0.97 & 0.92 \\
\hline $\operatorname{Sim} 4$ & correlated & yes & 5.2 & 2.8 & 1.2 & 0.9 & 0.46 & 0.43 & 0.93 & 0.94 \\
\hline
\end{tabular}

Note. ${ }^{a}$ Purity estimated at point source signal-to-noise threshold of 5 (3) in total (polarized) intensity.

This paper is dedicated to the memory of Andrew Lange, who gave wisdom and guidance to so many members of the astrophysics and cosmology community. His presence is sorely missed. We thank our colleagues on the BICEP experiment for useful discussions. QUaD is funded by the National Science Foundation in the USA, through grants ANT-0338138, ANT0338335, and ANT-0338238, by the Science and Technology Facilities Council (STFC) in the UK, and by the Science Foundation Ireland. We thank the staff of the AmundsenScott South Pole Station and all involved in the United States Antarctic Program for the superb support operation which makes the science presented here possible. Special thanks go to our intrepid winter scientist Robert Schwarz who spent three consecutive winter seasons tending the QUaD experiment. The BOOMERanG collaboration kindly allowed the use of their CMB maps for our calibration purposes. M.L.B. acknowledges the award of a PPARC Fellowship. S.E.C. acknowledges support from a Stanford Terman Fellowship. J.R.H. acknowledges the support of an NSF Graduate Research Fellowship and a Stanford Graduate Fellowship. C.P. acknowledges partial support from the Kavli Institute for Cosmological Physics through the grant NSF PHY-0114422. E.Y.W. acknowledges receipt of an NDSEG fellowship. J.M.K. acknowledges support from a John B. and Nelly L. Kilroy Foundation Fellowship.

\section{APPENDIX \\ SIMULATIONS}

The source extraction algorithm presented in Section 3 is tested using simulated distributions of point sources, with and without a toy model diffuse background. These simulations are used to determine the survey completeness, purity, the accuracy of recovered individual source parameters, the accuracy of recovered source distribution parameters, and the effect of choice of background smoothing kernel width $\sigma_{\text {bck }}$.

\section{A.1. Galactic Model Generation}

The methods described in Appendices A.1.1 and A.1.2 below are used to generate four types of galaxy simulation: random and correlated spatial distributions of point sources, with and without a diffuse background component-Table 4 summarizes the properties of each simulation.

\section{A.1.1. Simulated Source Populations}

Point sources are placed over the area of pixels occupied by the QUaD survey, using two methods to generate their spatial distribution. The first is a simple random distribution, denoted Sim1, with a partner simulation (Sim2) also constructed from the same spatial distribution of sources but with the inclusion of a diffuse background component (see Appendix A.1.2 below).
The second more closely matches the observed clustering of discrete sources. A two-point angular correlation function $w(\theta)=k \theta^{-\gamma_{c}}$ is used to model the clustering of sources and a power-law probability distribution function in Galactic latitude $b$ is simultaneously implemented to capture the observation that sources tend to be concentrated toward the Galactic plane, i.e., $p(b) d b \propto b^{-\beta} d b$. This simulation is called Sim3, and a further simulation, $\operatorname{Sim} 4$, is generated by taking the same point source population and adding a diffuse component. The power-law exponents $\gamma_{c}$ and $\beta$ are chosen such that the spatial distribution of sources qualitatively matches that in the QUaD data. Since it contains both a diffuse background and spatially clustered point sources, Sim4 is the model which most closely resembles the real data.

The physical properties of the sources are defined as follows: $100 \mathrm{GHz}$ total intensity source fluxes are drawn from a powerlaw model for the source counts $\left(d N / d S \propto S^{\gamma_{S}}\right.$, with $\gamma_{S}=$ -1.5 ) between 0.1 and $250 \mathrm{Jy}$; the normalization is chosen to match the average source density (i.e., number per square degree) in the QUaD I catalog. Spectral indices between 100 and $150 \mathrm{GHz}$ are generated using a Gaussian probability distribution function (pdf) of zero mean and unit width; $150 \mathrm{GHz}$ fluxes are generated by combining the spectral index and $100 \mathrm{GHz}$ flux. The polarization fraction for each source is a random number drawn uniformly between $0 \%$ and $20 \%$, while the polarization angle is also a uniform random number between $0^{\circ}$ and $180^{\circ}$.

\section{A.1.2. Simulated Diffuse Background}

Adding a model diffuse background allows its effect on recovered source properties to be assessed. This component is modeled using a weighted sum of a single point source map smoothed to different resolutions.

Point source locations are generated using the correlation function approach described in Appendix A.1.1 for Sim3 and Sim4, with a source density $\sim 20$ times higher than the real data. All sources have the same $100 \mathrm{GHz}$ fluxes $S_{\mathrm{bck}}$, a spectral index of 2.18 (the mean spectral index of the diffuse emission as calculated in the Map Paper), and a polarization fraction of $2 \%$ in pure $+Q$ in Galactic coordinates; these sources are placed within the boundaries of the survey in $I, Q$, and $U$ maps at each frequency $v$, giving a set of template maps $m_{t, v, i}$, where $i$ refers to each Stokes parameter.

To construct the model of diffuse emission, each $m_{t, v, i}$ is first smoothed with a set of circular Gaussian kernels of differing width $\sigma_{j}$ to yield smoothed maps $m_{t, v, i, j}$, with $-1.4 \leqslant$ $\log _{10}\left(\sigma_{j} / \mathrm{deg}\right) \leqslant 0.2$ in intervals of $\delta \log _{10}\left(\sigma_{j}\right)=0.2$. The smoothed maps are then co-added with a different weight $w_{j}$ for each smoothing kernel, with $w_{j} \propto j^{7 / 4}$, yielding the diffuse 

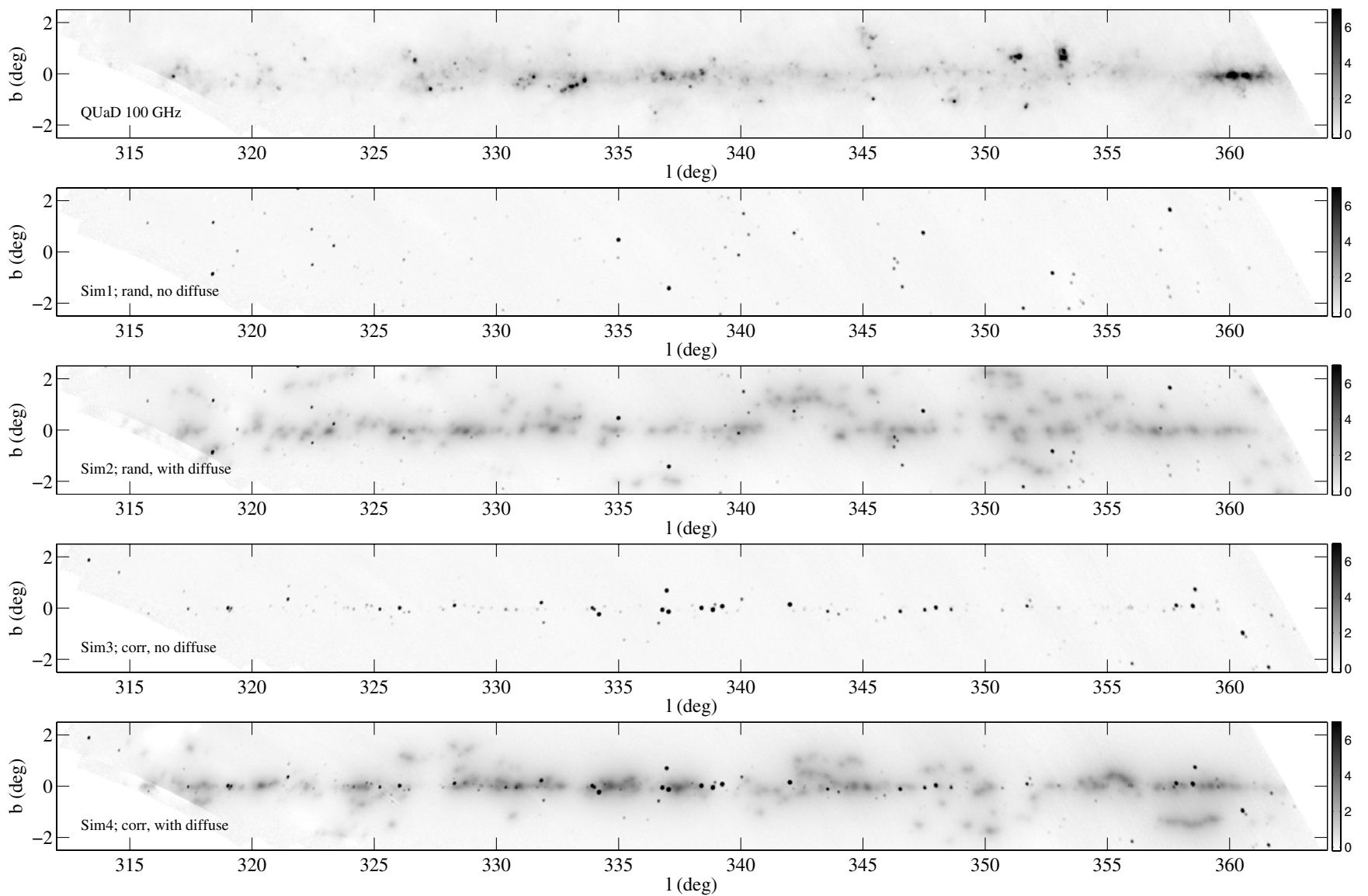

Figure 11. Comparison of QUaD $100 \mathrm{GHz}$ map to one realization of the simulations described in Appendix A.1.3, with all maps transformed to Galactic coordinates. The color scale is $\mathrm{MJy} \mathrm{sr}^{-1}$. From top to bottom: QUaD data, random point sources only, random sources plus diffuse background, spatially correlated sources, and spatially correlated sources plus diffuse background.

model map $m_{\mathrm{bck}, v, i}$ :

$$
m_{\mathrm{bck}, v, i}=\sum_{j} w_{j} m_{t, v, i, j}
$$

The $w_{j}$ amplitudes and $S_{\text {bck }}$ were chosen by requiring that the flux integrated over the whole simulated map matched that in the data within $\sim 10 \%$. A further consideration is that the relative weights between the $w_{j}$ and the exponent (defined somewhat arbitrarily here to be 7/4) should be chosen such that substructure on different angular scales in the model matches the data; a power spectrum analysis may represent the best way of determining the $w_{j}$ but is beyond the scope of this paper. Here, we merely note that with the choice of $\sigma_{j}$ and $w_{j}$ used above, the diffuse component of the simulations bear a qualitative resemblance to the data, as may be seen in Figure 11.

\section{A.1.3. Simulated Maps}

Having determined the positions and physical properties of the sources, they are then placed in a "source map," $m_{\text {src }}$, of the same size and pixel resolution as the QUaD maps. A similar map $m_{\text {bck }}$ is generated for the diffuse background following Appendix A.1.2; $m_{\text {src }}$ alone is the input sky for Sim1 and Sim3, with $m_{\text {src }}+m_{\text {bck }}$ used for Sim2 and Sim4. For each type of point source spatial distribution (random or correlated), the coordinates of each source are the same with and without a diffuse component present. This allows the effect of the background on source fluxes to be investigated separately from spatially correlated source positions. Note that a different realization of the diffuse component is used for the random and correlated source simulations, as may be seen in Figure 11.

Simulated detector timestream is interpolated from these maps using the pointing information from each day of real QUaD data and realistic noise added as described in the Map Paper. The simulated signal+noise data are then subjected to the same filtering and mapmaking steps as in the QUaD data pipeline described in the Map Paper. Figure 11 shows the QUaD $100 \mathrm{GHz}$ I map, and maps from one realization of each simulation type.

The maps are passed through the source extraction algorithm described in Section 3 to generate catalogs of sources in $I, Q$, and $U$ at the two QUaD frequencies. To determine quantities such as spectral index and polarization fraction (that is, quantities which require the catalog from more than one map), sources are matched using the simple spatial criterion described in Section 3. Many sky realizations are processed to build up sufficient statistics to characterize the survey depth and systematic effects.

\section{A.2. Completeness}

The completeness $C(>S)$, the fraction of input sources recovered above flux $S$, is shown in Figure 12 for the QUaD frequency bands for each set of simulations. The 90\% completeness limits (the flux $S_{90}$ at which the completeness reaches 90\%) for the simulated catalogs are summarized in Table 4.

The $90 \%$ completeness limit in total intensity is generally higher than in polarized intensity; this is in part due to the 

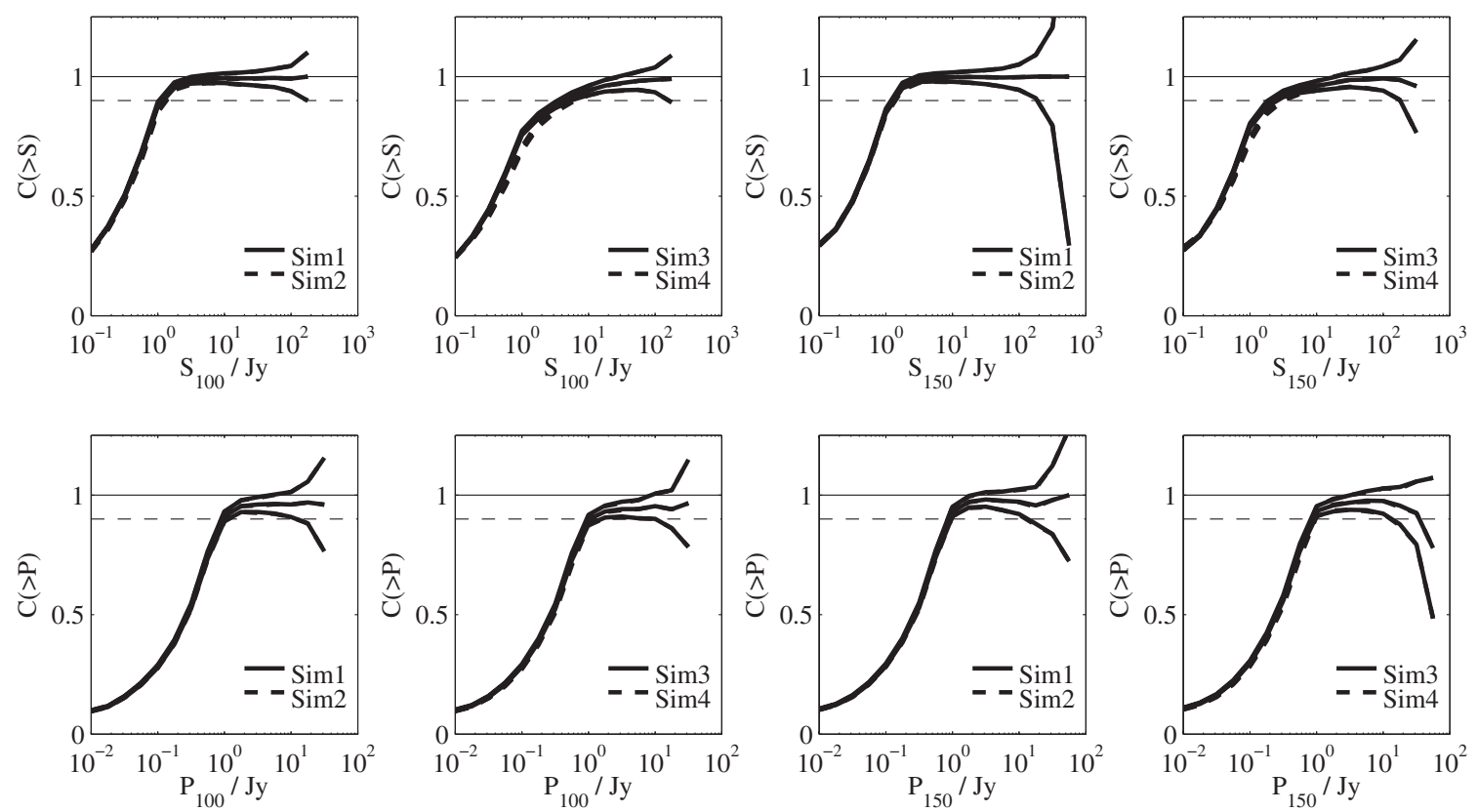

Figure 12. Completeness estimated from simulated point source distributions in total (top) and polarized (bottom) intensity. The left pair of columns is for $100 \mathrm{GHz}$, right pair for $150 \mathrm{GHz}$. Within each pair, the left column is for randomly distributed sources with and without a diffuse component, and likewise for spatially correlated sources in the right column. Solid black curves correspond to sources with no diffuse background present, dashed black curves are for the same source distributions with diffuse emission added. The contours indicate fluctuations in source counts for each flux bin calculated using Poisson statistics. The thin dashed black line indicates $90 \%$ completeness.

higher signal-to-noise detection threshold than that used in polarization (5 compared to 3 ). The limit in $I$ is also increased due to the shape of the completeness curve, where $C(>S)$ increases rapidly to 0.5 in $I$ but then approaches unity more slowly-see Figure 12. This effect is attributed to residual $1 / f$ noise in the $I$ map, rather than the diffuse background or source confusion, since it is present for all simulations. The completeness curves do not always asymptote to unity at high flux since sources can lie at map edges, where the map noise is much higher than the median, or on regions where the survey is shallower due to bad data or poor weather. In Sim1 and Sim2, the 90\% completeness limits for $I$ are 1.2 and $1.3 \mathrm{Jy}$, respectively, at $100 \mathrm{GHz}$, and 1.3 and $1.4 \mathrm{Jy}$ at $150 \mathrm{GHz}$. The 90\% limit is higher for Sim3, reaching 4.5 (2.3) Jy at 100 (150) GHz. Since this simulation contains no background, the effect is solely due to increased source confusion resulting from the spatial clustering of the sources. For Sim4, these numbers increase marginally to 5.2 and 2.8 at 100 and $150 \mathrm{GHz}$, respectively, indicating that the spatial clustering of sources causes a larger rise in completeness than the presence of a diffuse background.

In polarization, the diffuse background is faint enough that it does not strongly affect the completeness, while source clustering introduces only a small effect-all simulations indicate a $90 \%$ completeness polarized flux of 1-1.2 Jy at $100 \mathrm{GHz}$ and $0.9 \mathrm{Jy}$ at $150 \mathrm{GHz}$ - see Figure 12. The slightly higher $100 \mathrm{GHz}$ completeness is due to source confusion, since the randomly oriented polarization angles could result in polarized flux dilution, reducing the completeness. The survey coverage is smaller at $100 \mathrm{GHz}$ due to the smaller area of the QUaD focal plane at this frequency (the $100 \mathrm{GHz}$ survey is approximately $7 \%$ smaller in polarization than $150 \mathrm{GHz}$ ). As a result, some input sources may not lie in the $100 \mathrm{GHz}$ survey area, explaining why the completeness curve does not asymptote to unity at this frequency.

\section{A.3. Purity}

The "purity" of the survey $p$ is the number of recovered sources which were matched spatially to the input catalog divided by the total number of recovered sources. This is quantified by comparing the input and recovered source catalogs in Sims1-4. Figure 13 shows the purity as a function of signal-to-noise threshold in both total and polarized intensity. The values of $p$ at the chosen extraction thresholds $(\mathrm{S} / \mathrm{N}>5$ in $I, \mathrm{~S} / \mathrm{N}>3$ in $P$ ) are summarized in Table 4.

Between detection thresholds of $2<\mathrm{S} / \mathrm{N}<8$, the purity increases more rapidly with $\mathrm{S} / \mathrm{N}$ in both total and polarized intensity if the spatial distribution of sources is correlated rather than random. This is due to flux boosting of a source by fainter, spatially coincident sources which are not resolved themselves. In total intensity, the purity appears poor $(40 \%-50 \%)$ when the diffuse background is present (Sim2 and Sim4), even at a detection threshold of $\mathrm{S} / \mathrm{N}>12$. However, these "false detections" are not noise fluctuations. Investigation of the output catalogs from Sim2 and Sim4 demonstrated that the detected sources unmatched to the input catalog were beam scale or extended sources associated with substructure in the simulated diffuse background. Figure 14 illustrates this effect for a Sim4 realization, demonstrating how substructure can be erroneously detected as real discrete sources. This effect makes "purity" an ambiguous concept in the context of separating sources from a diffuse background with enough power on beam-sized angular scales. If the signal-to-noise of the diffuse background is comparable to that of the discrete sources, the purity is not dominated by noise fluctuations, but the inability to distinguish the sources of interest from resolved substructure in the diffuse emission. Simulations Sim2 and Sim4 fall into this category, which is why their purity is low even at high signal-to-noise thresholds; this effect is simply a consequence of the model parameters chosen in Appendix A.1.2. As shown in Section 4, 

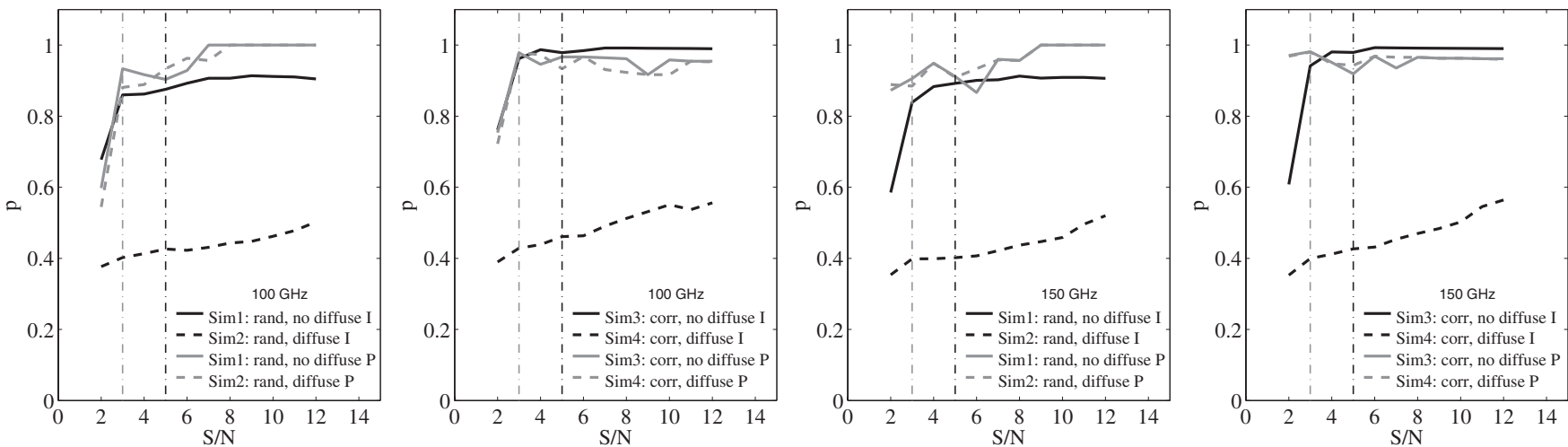

Figure 13. Purity as estimated from simulated point source distributions as a function of $\mathrm{S} / \mathrm{N}$. The left (right) pair of columns correspond to purity at 100 (150) GHz. Within each pair, the left plot is for randomly distributed sources with and without a diffuse background (Sim1 and Sim2), and the right plot similar but for spatially correlated sources (Sim3 and Sim4). Survey purity in total (polarized) intensity is plotted in black (gray). The vertical black dot-dashed lines correspond to the S/N ratio used to extract sources in $I$; the vertical gray dot-dashed line is the same for polarization.

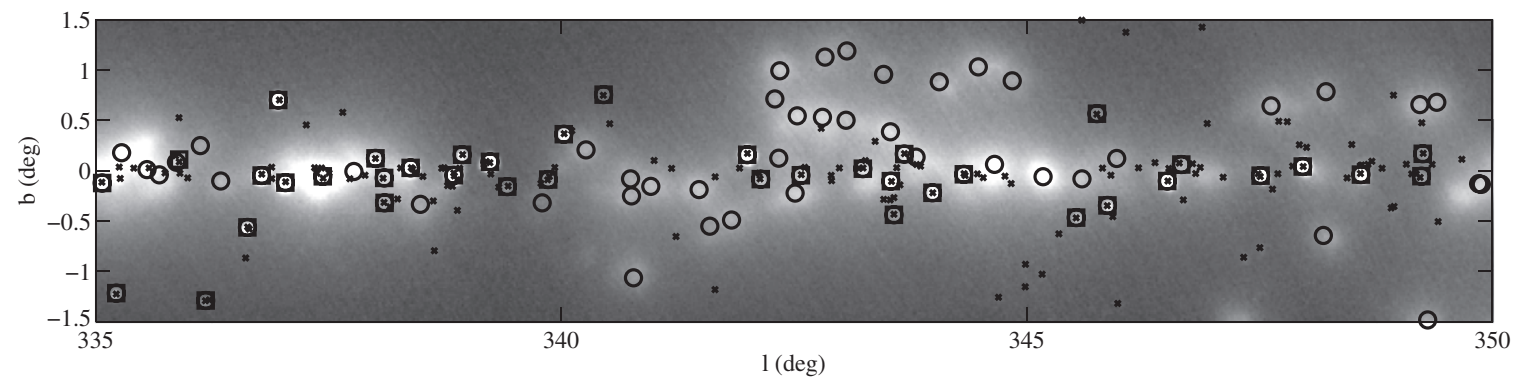

Figure 14. Illustration of purity in the presence of diffuse background. The image is a subsection of the Sim $4100 \mathrm{GHz} I$ map shown in the bottom panel of Figure 13 . Crosses correspond to the locations of input sources, squares are the sources detected from Sim3, and circles are the sources detected in Sim4 (recall that the input point source populations are identical for Sim3 and Sim4). In Sim4, false sources are detected from substructure in the diffuse emission-these sources have a circle that does not enclose a cross. This systematic effect is the cause of the low purity for simulations which have a diffuse background present, as described in Appendix A.3.

$80 \%(55 \%)$ of the QUaD sources detected in total intensity at 100 (150) GHz have IRAS-PSC counterparts. This indicates that the QUaD catalog may contain such impurities, though we may not expect all QUaD sources to have an IRAS counterpart due to, e.g., differing resolution between the experiments and incomplete knowledge of source emission spectra. The true purity of the catalog likely lies between the results of $\operatorname{Sim} 3$ and Sim4, with the purity of the former indicating the catalog limitations due to instrumental noise and source clustering, and the latter showing the influence of diffuse emission detected as beamscale compact sources. A power-spectrum approach, advocated in Appendix A.1.2, represents the best way to determine the amount power on different angular scales.

The diffuse polarized background is faint enough that there is little difference between the purity in each type of simulation. Figure 13 shows that a polarized source detection threshold of $\mathrm{S} / \mathrm{N}>3$ results in a catalog that is $\sim 90 \%$ pure for randomly distributed sources or $\sim 100 \%$ pure for spatially correlated sources.

\section{A.4. Source Recovery}

Recovered catalogs from the four sets of simulations are used to estimate how accurately the input parameters of individual sources and source distributions can be recovered. While the output distributions for $\operatorname{Sim} 1$ and $\operatorname{Sim} 3$ (those without a diffuse background) should be insensitive to the choice of input distribution parameters, such as the source counts slope $\gamma_{S}$, the systematic biases introduced by the background (simulations Sim2 and $\operatorname{Sim} 4$ ) do depend on the background model parameters $\gamma_{c}$, $\beta, w_{i}, \sigma_{i}$, and the overall amplitude. We therefore caution that while the toy model of the diffuse component allows a qualitative impression of how source properties can be corrupted, the amount of corruption depends on these parameters to an extent that may differ from the real data.

\section{A.4.1. Recovery of Individual Source Parameters}

Figure 15 compares the recovered source properties to their input values. In total flux $S$, we show the $16 \%, 50 \%$, and $84 \%$ percentiles of the ratio $S_{\text {out }} / S_{\text {in }}$, while the same percentiles are shown for polarization fraction difference $f_{\text {out }}-f_{\text {in }}$ and polarization angle difference $\phi_{\text {out }}-\phi_{\text {in }}$. Both $100 \mathrm{GHz}$ and $150 \mathrm{GHz}$ simulations are shown, though similar behavior is observed at both frequencies.

For total intensity $S$, in all simulations the median $S_{\text {out }} / S_{\text {in }}$ falls as sources get fainter, but is within a few percent of unity down to $10 \mathrm{Jy}$ (comparable to the absolute calibration uncertainty of $3.5 \%$ ). At this flux, the $16 \%$ and $84 \%$ percentiles of $S_{\text {out }} / S_{\text {in }}$ are $\sim 5 \%$ from the median for simulations without a diffuse background (Sim1 and Sim3). Below $10 \mathrm{Jy}$, rather than more numerous faint sources being boosted to higher fluxes, the systematic deficit in recovered flux is due to filtering of the timestream before map co-addition (see the Map Paper); this demonstrates that filtering effects are more important than flux boosting due to instrumental noise at low fluxes.

Including diffuse emission results in a wider and more asymmetric distribution of recovered flux due to background contamination; at $10 \mathrm{Jy}$ in Sim3 (no diffuse emission), the 

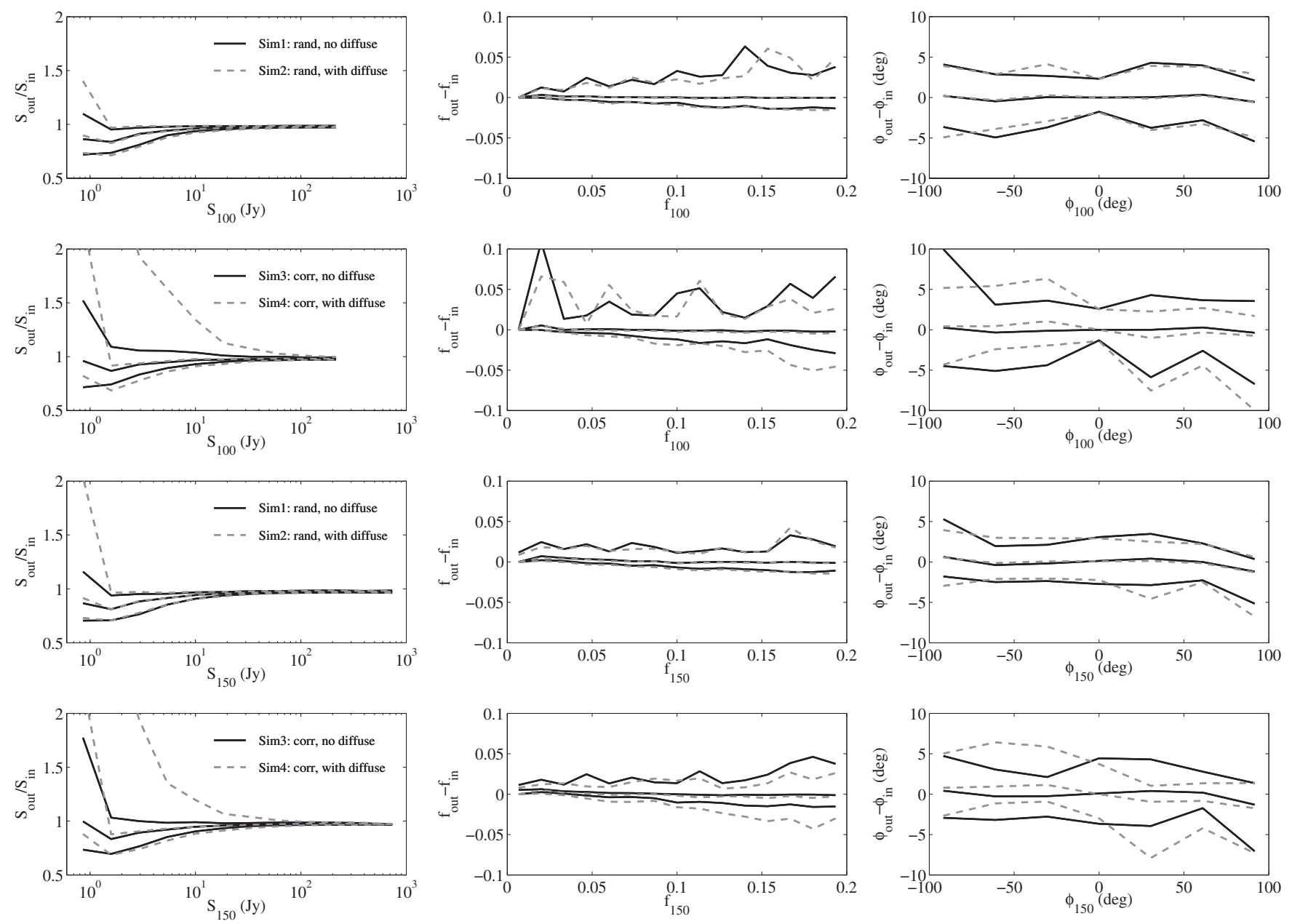

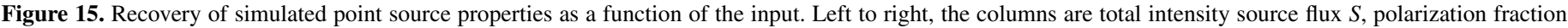

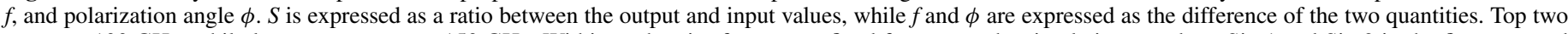

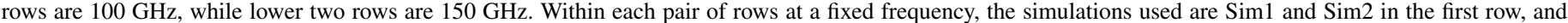

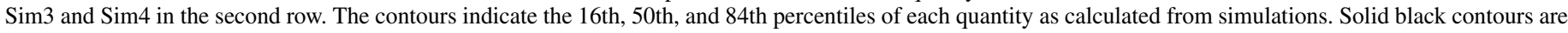
for simulations without a diffuse background (Sim1 and Sim3), while gray dashed contours indicate the simulations with diffuse emission (Sim2 and Sim4).

systematic uncertainties are $\sigma_{-}=0.04$ and $\sigma_{+}=0.07$, while the same quantities in Sim4 (including diffuse emission) are $\sigma_{-}=0.07$ and $\sigma_{+}=0.36$, where $\sigma_{-}$is the difference between the 50th and 16th percentiles of the ratio $S_{\text {out }} / S_{\text {in }}$ at $10 \mathrm{Jy}$, and $\sigma_{+}$is the difference between the 84th and 50th percentiles. The asymmetric errors due to background contamination are discussed further in A.4.2 in the context of the source counts, $d N / d S$.

The recovery of the point source polarization fraction is not strongly biased in any of the simulations. The median difference between input and output polarization fractions is $<1 \%$, at which point beam systematic effects become important (see Instrument Paper for details). The scatter on $f_{\text {out }}-f_{\text {in }}$ generally increases with higher polarization fraction, rising from $\sim 1 \%$ at $f \sim 1 \%$ to $5 \%$ for $f=20 \%$. This is because only the brightest sources tend to be detected at low $f$, while at high $f$ both bright and faint sources are included, increasing the variance of $f_{\text {out }}-f_{\text {in }}$. The distribution of $f_{\text {out }}-f_{\text {in }}$ is skewed toward positive values; this effect is attributed to the addition of noise to the total polarized flux as $\sigma_{P}^{2}=\sigma_{Q}^{2}+\sigma_{U}^{2}$. No systematic bias is introduced when a diffuse component is present, indicating that the background removal strategy is effective for determining fluxes of polarized sources.
One might ask why the recovered $f$ is not systematically lower than the input, since the polarization angles assigned to simulated sources are random and therefore should average to zero when sources are confused, as in Sim3 and Sim4? The reason is that the simulations use a power-law distribution of fluxes, resulting in many more faint sources per unit solid angle than bright sources. In a given resolution element, faint sources will largely cancel each others' polarized flux, while a statistically unlikely (but far brighter) polarized source will dominate the polarized emission.

The simulations show that polarization angle difference $\phi_{\text {out }}-\phi_{\text {in }}$ suffers a systematic shift of $<1^{\circ}$, with 16 and 84 percentiles less $<5^{\circ}$. The scatter in $\phi$ is not strongly affected by a diffuse component, but increases marginally when sources are spatially correlated. This increased scatter is due to confusion of sources with random polarization angles within a single beam element; although on average fainter sources with random $\phi$ will average to zero, their presence will introduce extra fluctuation into the polarization angle of the brightest source.

\section{A.4.2. Recovery of Source Counts}

Of particular interest to source surveys are the source counts $d N / d S$; it is therefore important to address whether this quantity 

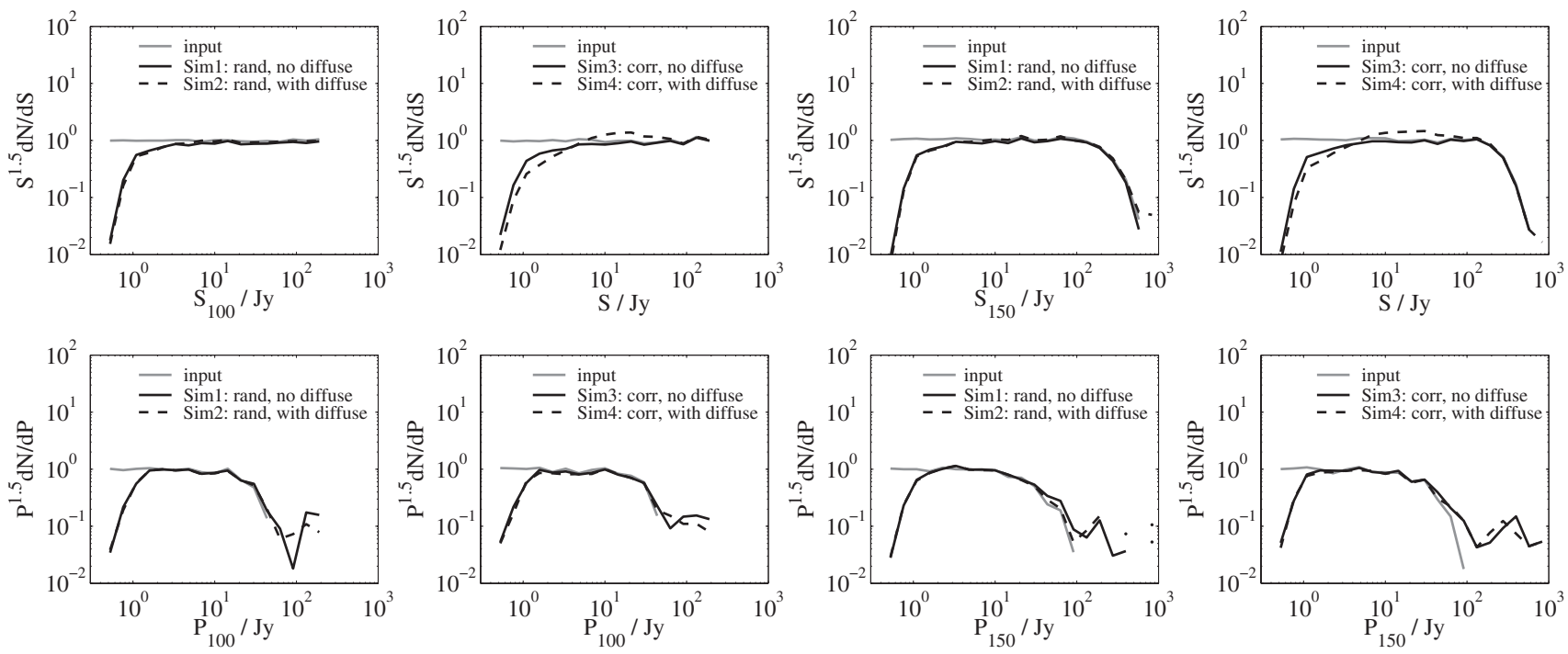

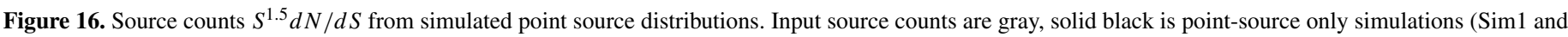

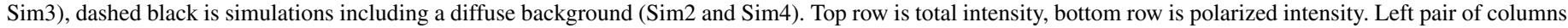

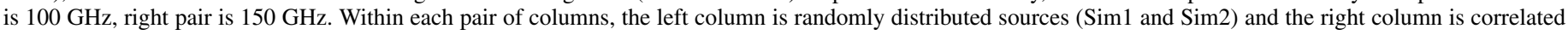

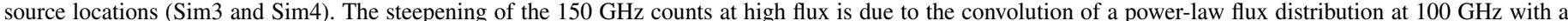
Gaussian spectral index distribution.

can be accurately recovered in the presence of noise and a diffuse background. Figure 16 shows $S^{1.5} d N / d S$ as a function of source flux, for the input and recovered source distributions, in each of the simulation types Sim1-Sim4. Since the input counts were $d N / d S \propto S^{-1.5}$, in this plot perfectly recovered counts appear as a line of zero gradient. The figure shows that for Sim1-Sim3, the counts obey the expected property of being well recovered at high flux, but falling off as the flux approaches the survey detection threshold. Note that the falloff at high flux at $150 \mathrm{GHz}$ is due to the finite maximum $100 \mathrm{GHz}$ source flux convolved with the assumed spectral index distribution; this part of the input counts does not obey a power law but is well recovered by the source extraction. The source counts show similar properties at both frequencies, in total and polarized intensity.

Only in the case of the total intensity fluxes of clustered sources in the presence of a diffuse background (Sim4) do we see significant deviations from the ideal behavior; in this case, boosting occurs from low fluxes to higher fluxes. This may be seen from the deficit of sources at low flux relative to simulations without a diffuse component and an excess above the input counts at high fluxes. In the high flux regime, where source counts are typically fit, the shape of $S^{1.5} d N / d S$ is heavily distorted from a simple power law, rendering constraints on this quantity difficult to measure. Note the distinction between this type of flux boosting, which is due to background emission, and that due to uniform survey noise; in extragalactic surveys of radio sources, only the latter is normally considered (e.g., Muchovej et al. 2010), and can be corrected by marginalizing over the underlying source count parameters. For the former, which is called "background boosting" here, de-boosting source fluxes is not an easy problem. While diffuse emission such as the CMB is very well characterized as a Gaussian random noise distribution (and could potentially also be marginalized over to find the true source flux), the properties of the diffuse Galactic emission (such as morphology, spectral behavior, and projection effects along different lines of sight through the Galaxy) are at present poorly constrained and not easy to model. Contamination by Galactic emission is also asymmetric in the sense that sources are only ever boosted to higher fluxes, because unlike CMB fluctuations (unpolarized) Galactic emission is always positive.

We therefore caution against overinterpretation of the source count slope in the presence of a Galactic "background," since the slope is shown by simulations to be corrupted despite the aggressive background filtering. The level of corruption is dependent on the parameters of the diffuse background model (such as the amount of power in beam-scale substructure). As we only use one set of parameters in the simulations, the results presented above are not intended to precisely quantify this systematic error, but to explore how the source counts can be affected by the background in a restrictive region of diffuse model parameter space. Despite these limitations, the source counts of the QUaD survey data shown in Figure 5 do appear to obey a power law at high fluxes, indicating that in this regime background contamination is likely unimportant.

\section{A.4.3. Recovery of Spectral Index Distribution}

Figure 17 shows the recovered total intensity spectral index probability distribution $\operatorname{Pr}\left(\alpha_{I}\right)$ for one realization of each simulation type. The input distribution, a Gaussian probability distribution function of unit rms, is well recovered for randomly distributed and spatially correlated sources without a diffuse background (Sims 1 and 3). Sims 2 and 4 exhibit a small shift of the distribution center toward larger values; this is likely due to contamination of faint source fluxes by the diffuse emission. In support of this notion, the shift is larger for Sim4 than Sim2 since more sources lie close to the plane of the Galaxy, where diffuse emission is brightest.

We conclude that the recovered spectral index distribution from the QUaD data (Section 4.4) may be biased slightly high as a result of background contamination preferentially affecting the $100 \mathrm{GHz}$ data. However, the degree to which $\operatorname{Pr}(\alpha)$ is corrupted in simulations depends on the diffuse model input parameters; we therefore refrain from quantifying the effect.

\section{A.4.4. Recovery of Correlation Function}

Simulations Sim3 and Sim4 are used to test the recovery of the input source spatial distribution parameters for sources clus- 

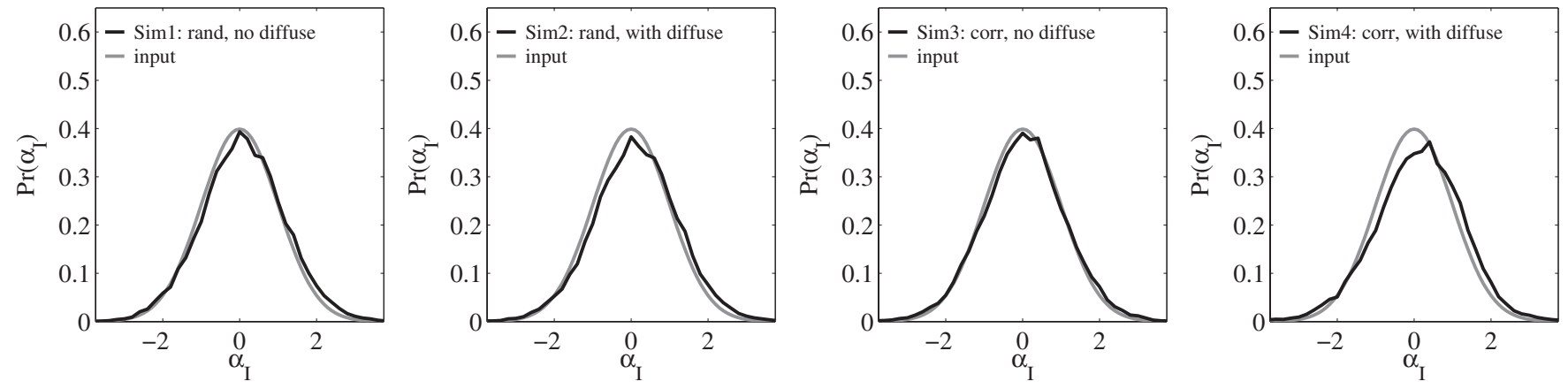

Figure 17. Recovered total intensity source spectral index distribution shown in black for (left to right) Sim1, Sim2, Sim3, and Sim4, compared to input (gray).
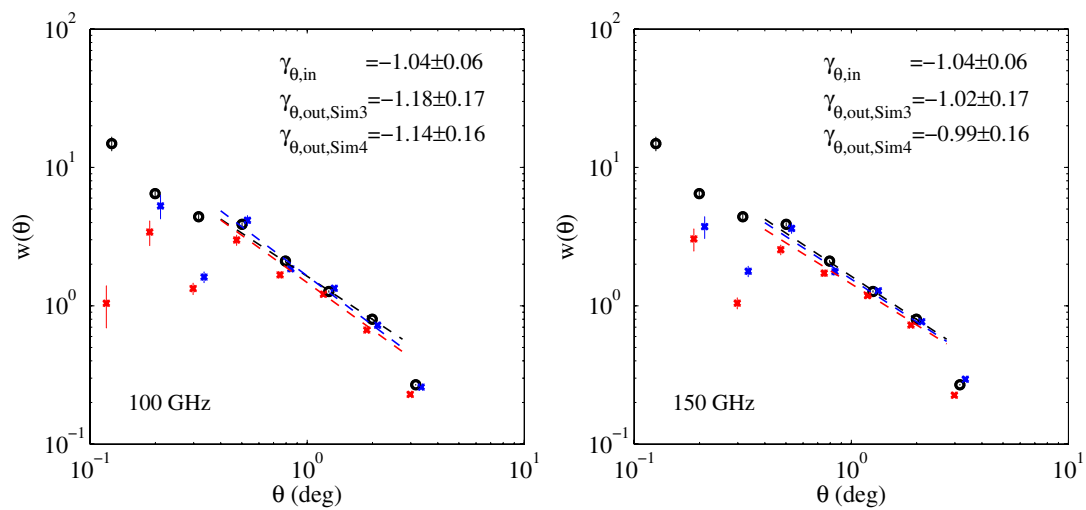

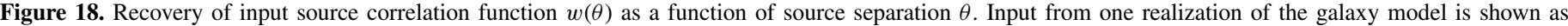

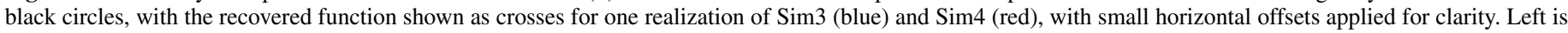

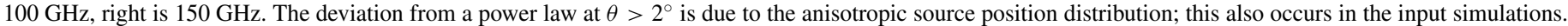

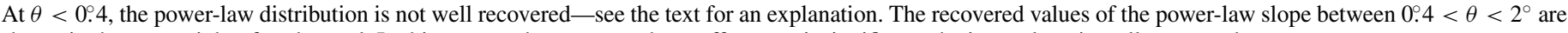
shown in the upper right of each panel. In this range, where survey shape effects are insignificant, the input slope is well recovered.

(A color version of this figure is available in the online journal.)

tered in the plane of the Galaxy, as described in Section 4.5. The angular correlation function is constructed as in Equation (4), except this time $H_{d}(\theta)$ (the number of sources with a neighbor at separation $\theta$ ) is derived from a realization of $\operatorname{Sim} 3$ or Sim4, depending on whether a diffuse background is included. As with the real data, Sim1 is used to generate a histogram of the number of randomly distributed sources with a neighbor at separation $\theta, H_{r}(\theta)$. Correlation functions are generated for clustered point sources with and without a diffuse background present; the results for a single simulated realization are presented in Figure 18.

The black input points show that while a power-law correlation function is traced at small $\theta$, at large source separations the slope becomes steeper. This is a result of the anisotropic nature of the source distribution; less than $1 \%$ of sources at each frequency lie further than $3^{\circ}$ from the plane, and those inside are preferentially located toward $b=0$ (see Section 4.2). Only source clustering in Galactic longitude contributes to the probability of finding a source separation greater than $\sim 3^{\circ}$, suppressing the correlation function at large angular scales. The reconstructed $w(\theta)$ from the simulated data shows this effect, demonstrating that although the correlation function is intrinsically suppressed due to the distribution of sources in the galaxy, it is still well recovered at large angular separations. At angular scales $<0.4, w(\theta)$ becomes poorly recovered. This is due to the large probability that the closest neighbors to bright, rare sources are faint and likely below the detection threshold. Therefore $w(\theta)$ is only well recovered when the source separation is large enough that the probability of a bright neighboring source is significant.
Figure 18 shows that the slope of the correlation function is well recovered in the range $0.4<\theta<2^{\circ}$ to within the uncertainties; this is the range chosen for fitting $w(\theta)$ in the QUaD data (Section 4.5).

\section{A.5. Effect of Background Kernel $\sigma_{\mathrm{bck}}$}

Without removal of the diffuse background, the measured flux of each extracted source in the QUaD survey can be heavily influenced by proximity to other bright sources and/or diffuse emission; either can add excess signal when source fluxes are determined, biasing recovered fluxes high. The filtering scheme described in Section 3 is designed to suppress the background by subtracting a template of diffuse emission from the maps. The template is a smoothed version of the raw survey map, with point source pixels replaced by their local median. Constructing the template requires a choice of smoothing scale $\sigma_{\text {bck }}$, which represents the minimum angular scale on which background fluctuations are assumed significant. The results from the QUaD survey and the simulations in Appendices A.2-A.4 are dependent on the choice of $\sigma_{\text {bck }}$; here we investigate the effect of varying the value of this parameter.

Figure 19 shows the ratio of recovered $100 \mathrm{GHz} I$ fluxes to the input as a function of $\sigma_{\text {bck }} / \sigma_{\text {beam }}$ for sources above $25 \mathrm{Jy}$ (similar results are found at $150 \mathrm{GHz}$ ). The left panel shows that for Sim1, source fluxes are recovered to within $\sim 2 \%$ if $\sigma_{\text {bck }} / \sigma_{\text {beam }}>3$, with a deficit of $5 \%$ by $\sigma_{\text {bck }} / \sigma_{\text {beam }}=2$. A similar result is found for $\operatorname{Sim} 2$, though increased variance is found for $\sigma_{\text {bck }} / \sigma_{\text {beam }}>3$. The systematic reduction of flux at low $\sigma_{\text {bck }} / \sigma_{\text {beam }}$ is due to source flux being subtracted with the background as $\sigma_{\text {bck }} \rightarrow \sigma_{\text {beam. }}$. Increased variance is 

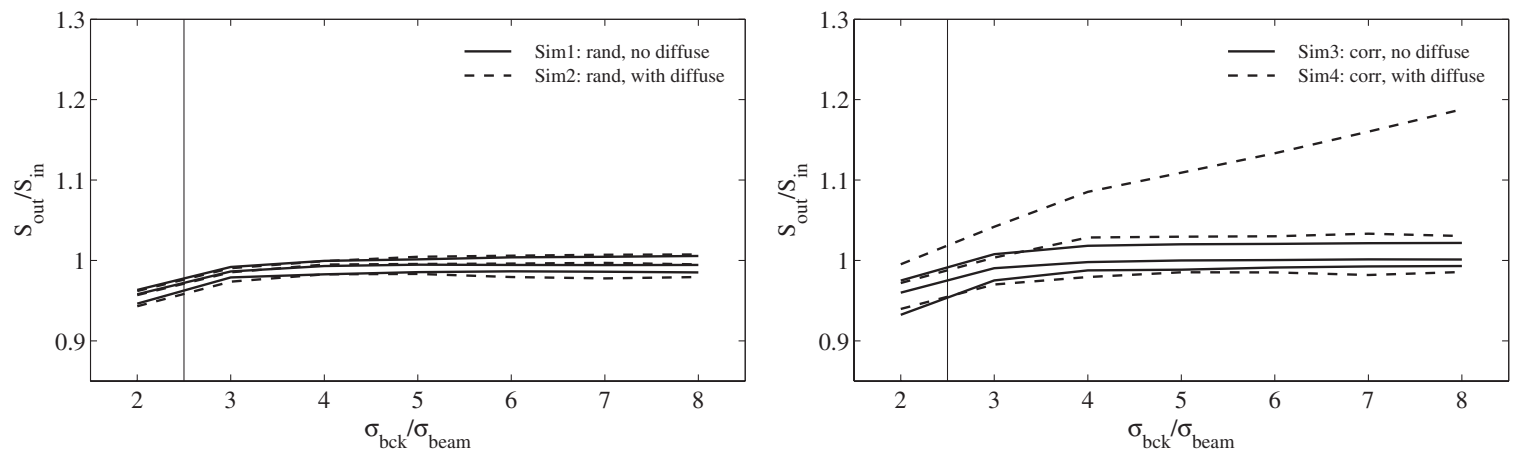

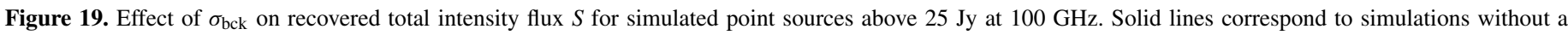

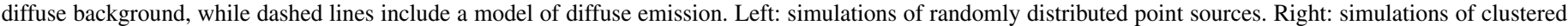

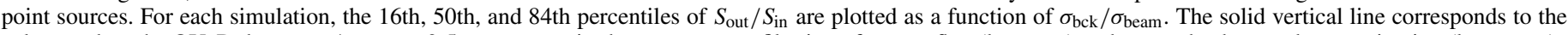

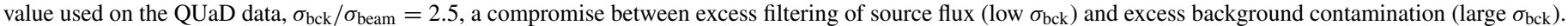

found when source locations are correlated, as in Sim3 (see right panel of Figure 19). Since this simulation is devoid of background emission, the larger 84th percentile is due to source confusion, as the likelihood of more than one source per beam is larger for spatially correlated sources. As might be expected, the 16th percentile is not significantly changed compared to randomly distributed sources since confusion cannot reduce recovered source fluxes. We note that despite source confusion, fluxes are still generally recovered to within $5 \%$ or better, independent of $\sigma_{\text {bck }}$. Including a diffuse background (Sim4) results in variance of $S_{\text {out }} / S_{\text {in }}$ which increases as a function of $\sigma_{\text {bck }} / \sigma_{\text {beam }}$. The variance is skewed toward positive fluctuations due to the positive signal from the diffuse emission, which increasingly contributes to $S_{\text {out }}$ as $\sigma_{\text {bck }}$ rises (because less diffuse emission is removed in the background subtraction stage). By $\sigma_{\text {bck }} / \sigma_{\text {beam }}=8$, the 84 th percentile of $S_{\text {out }} / S_{\text {in }}$ is $\sim 1.2$, compared to the 50th percentile of $\sim 1.05$; the fluctuation toward larger values of $S_{\text {out }} / S_{\text {in }}$ is therefore $\sigma_{+}=0.15 \%$ or $15 \%$ higher than the input value. It is likely that this effect is also present in Sim2, where a diffuse component is present and sources are randomly distributed, but since far fewer sources are located close to the Galactic plane, where the background is brighter, the effect is less obvious.

The choice of $\sigma_{\text {bck }}$ is therefore a compromise between loss of source flux due to excessive background subtraction (smaller $\sigma_{\text {bck }}$ ) and increasing contamination from diffuse emission (larger $\left.\sigma_{\text {bck }}\right)$. Adopting $\sigma_{\text {bck }} / \sigma_{\text {beam }}=2.5$ as in the QUaD survey data results in $<5 \%$ systematic loss of source flux, while reducing the scatter due to the background to $\sim 5 \%$-both these effects are comparable to the absolute calibration uncertainty in the maps of $3.5 \%$.

\section{REFERENCES}

Agol, E. 2000, ApJ, 538, L121

Aitken, D. K., Greaves, J., Chrysostomou, A., Jenness, T., Holland, W., Hough, J. H., Pierce-Price, D., \& Richer, J. 2000, ApJ, 534, L173

Altenhoff, W. J., Downes, D., Pauls, T., \& Schraml, J. 1979, A\&AS, 35, 23

André, P., et al. 2010, A\&A, 518, L102

Bertin, E., \& Arnouts, S. 1996, A\&AS, 117, 393

Brown, M. L., et al. 2009, ApJ, 705, 978

Condon, J. J., Griffith, M. R., \& Wright, A. E. 1993, AJ, 106, 1095

Crawford, T. M., Switzer, E. R., Holzapfel, W. L., Reichardt, C. L., Marrone, D. P., \& Vieira, J. D. 2010, ApJ, 718, 513

Culverhouse, T. L., et al. 2010, ApJ, 723, L78

Désert, F.-X., et al. 2008, A\&A, 481, 411
Dickinson, C., Davies, R. D., Bronfman, L., Casassus, S., Davis, R. J., Pearson, T. J., Readhead, A. C. S., \& Wilkinson, P. N. 2007, MNRAS, 379, 297

Dowell, C. D. 1997, ApJ, 487, 237

Dowell, C. D., Hildebrand, R. H., Schleuning, D. A., Vaillancourt, J. E., Dotson, J. L., Novak, G., Renbarger, T., \& Houde, M. 1998, ApJ, 504, 588

Enoch, M. L., Evans, N. J., II, Sargent, A. I., Glenn, J., Rosolowsky, E., \& Myers, P. 2008, ApJ, 684, 1240

Enoch, M. L., et al. 2006, ApJ, 638, 293

Furukawa, N., Dawson, J. R., Ohama, A., Kawamura, A., Mizuno, N., Onishi, T., \& Fukui, Y. 2009, ApJ, 696, L115

Greaves, J. S., Holland, W. S., Murray, A. G., \& Nartallo, R. 1995, MNRAS, 272, L1

Hamaker, J. P., \& Bregman, J. D. 1996, A\&AS, 117, 161

Hennemann, M., et al. 2010, A\&A, 518, L84

Hildebrand, R. H. 1988, QJRAS, 29, 327

Hinderks, J. R., et al. 2009, ApJ, 692, 1221

Hinz, J. L., Rieke, G. H., Yusef-Zadeh, F., Hewitt, J., Balog, Z., \& Block, M. 2009, ApJS, 181, 227

Jones, D. I., Crocker, R. M., Ott, J., Protheroe, R. J., \& Ekers, R. D. 2011, AJ, 141,82

Jones, W. C., Bhatia, R., Bock, J. J., \& Lange, A. E. 2003, Proc. SPIE, 4855, 227

Juvela, M., et al. 2010, A\&A, 518, L93

Law, C. J., Yusef-Zadeh, F., Cotton, W. D., \& Maddalena, R. J. 2008, ApJS, 177,255

Leitch, E. M., et al. 2002, ApJ, 568, 28

Lis, D. C., \& Carlstrom, J. E. 1994, ApJ, 424, 189

Macquart, J., Bower, G. C., Wright, M. C. H., Backer, D. C., \& Falcke, H 2006, ApJ, 646, L111

Marrone, D. P., Moran, J. M., Zhao, J., \& Rao, R. 2007, ApJ, 654, L57

Masi, S., et al. 2006, A\&A, 458, 687

Melia, F., Liu, S., \& Coker, R. 2001, ApJ, 553, 146

Muchovej, S., et al. 2010, ApJ, 716, 521

Netterfield, C. B., et al. 2009, ApJ, 707, 1824

Novak, G., Dotson, J. L., Dowell, C. D., Goldsmith, P. F., Hildebrand, R. H., Platt, S. R., \& Schleuning, D. A. 1997, ApJ, 487, 320

Olmi, L., et al. 2009, ApJ, 707, 1836

Pilbratt, G. L., et al. 2010, A\&A, 518, L1

Quataert, E., \& Gruzinov, A. 2000, ApJ, 545, 842

Reich, W., Sofue, Y., \& Matsuo, H. 2000, PASJ, 52, 355

Rosolowsky, E., et al. 2010, ApJS, 188, 123

Schuller, F., et al. 2009, A\&A, 504, 415

Vieira, J. D., et al. 2010, ApJ, 719, 763

Villa, F., et al. 2002, in AIP Conf. Ser. 616, Experimental Cosmology at Millimetre Wavelengths, ed. M. de Petris \& M. Gervasi (Melville, NY: AIP), 224

Williams, J. P., Blitz, L., \& McKee, C. F. 2000, in Protostars and Planets IV, ed. V. Mannings, A. P. Boss, \& S. S. Russell (Tucson, AZ: Univ. Arizona Press), 97

Wood, D. O. S., \& Churchwell, E. 1989a, ApJ, 340, 265

Wood, D. O. S., \& Churchwell, E. 1989b, ApJS, 69, 831

Young, K. E., et al. 2006, ApJ, 644, 326

Yusef-Zadeh, F., Hewitt, J. W., \& Cotton, W. 2004, ApJS, 155, 421

Yusef-Zadeh, F., Morris, M., Slee, O. B., \& Nelson, G. J. 1986, ApJ, 310, 689 